\title{
Notes on the Hopkins-Miller Theorem
}

\author{
Charles Rezk
}

\begin{abstract}
We give an exposition of the proof of a theorem of Hopkins and Miller, that the spectra $E_{n}$ admit an action of the Morava stabilizer group.
\end{abstract}

\section{Introduction}

These expository notes were originally written as an aid to the author while he was giving a course on the proof of the Hopkins-Miller theorem (Theorem 2.1) at Northwestern in Spring 1997. The aim was to describe the theorem, and give a sketch of the proof.

The aim was achieved, though only by glossing over certain points. In particular, several places rely on the existence of closed model category structures on various categories of spectra, and on subtle properties of this structure; I have not tried to deal with these issues. A few other points are left as references. At some points, the exposition is rather terse. Otherwise, the proof is as complete as I could make it.

The matter of the final part of these notes, on the construction of $\mathcal{A}_{\infty}$-ring structures, has to some extent been superseded by recent work of Hopkins and Goerss. They have developed more powerful obstruction machinery for constructing rigid algebraic structures on spectra, and are able to prove results about the existence of $\mathcal{E}_{\infty}$-structures as well as of $\mathcal{A}_{\infty}$-structures.

The material is based primarily on lectures and courses given by Mike Hopkins which I have attended at one time or another, and on discussions with Mike Hopkins and Haynes Miller. Also of great use were notes prepared by Lee Nave at a course given by Mike Hopkins at the University of Washington in the Spring of 1996.

All errors are mine, most of the true bits belong to others.

1.1. Organization. After giving a statement of the main result in Section 2, we outline Lubin-Tate's theory of universal deformations of formal groups over fields of positive characteristic, and its relation to homotopy theory, in Sections $3-7$. In Sections $8-14$ we summarize what we need about the theory of spectra and $\mathcal{A}_{\infty}$-ring spectra, and set up an obstruction theory and a spectral sequence for calculating maps between $\mathcal{A}_{\infty}$-ring spectra. In Sections $15-21$ we give the calculation of the obstruction groups and the $E_{2}$-term of the spectral sequence, assuming that we have an $\mathcal{A}_{\infty}$-ring spectrum structure on the spectra of interest; the obstruction groups vanish and the spectral sequence collapses for algebraic

1991 Mathematics Subject Classification. Primary 55N22; Secondary 55P42, 55S99. 
reasons. Finally, in Sections 22-23 we outline an obstruction theory for constructing an $\mathcal{A}_{\infty}$-ring spectrum structure, and compute the obstruction groups by reducing to the calculations already carried out.

\section{The theorem}

In this section we state the main theorem, modulo definitions of some of the key concepts. (Formal group laws are defined in Section 3, universal deformations of formal group laws are defined in Section 4 , and $\mathcal{A}_{\infty}$-ring spectra are defined in Section 12.)

Let $\mathcal{F G}$ denote the category having as objects pairs $(k, \Gamma)$, where $k$ is a perfect field of finite characteristic $p$, and $\Gamma$ is a formal group of height $n$ over $k$, and with morphisms $\alpha:\left(k_{1}, \Gamma_{1}\right) \rightarrow\left(k_{2}, \Gamma_{2}\right)$ consisting of a pair $(i, f)$, where $i$ is a map $i: k_{2} \rightarrow k_{1}$ of rings and $f$ is an isomorphism $f: \Gamma_{1} \rightarrow i^{*} \Gamma_{2}$ of formal group laws.

TheOREM 2.1 (Hopkins-Miller). There exists a functor $(k, \Gamma) \mapsto E_{k, \Gamma}$ from $\mathcal{F} \mathcal{G}^{\mathrm{op}}$ to the category of $\mathcal{A}_{\infty}$-ring spectra, such that

1. $E_{k, \Gamma}$ is a commutative ring spectrum,

2. there exists a unit in $\pi_{2} E_{\Gamma}$,

3. $\pi_{o d d} E_{k, \Gamma}=0$, from which it follows that $E_{k, \Gamma}$ is complex orientable,

4. and such that the corresponding formal group law over $\pi_{0} E_{\Gamma}$ is the universal deformation of $(k, \Gamma)$.

In particular, when $(k, \Gamma)=\left(\mathbb{F}_{p^{n}}, F_{n}\right)$ where $F_{n}$ is the unique $p$-typical formal group law of height $n$ with $[p]_{\Gamma}(x)=x^{p^{n}}$, then $E_{k, \Gamma}$ is called $E_{n}$, and

$$
\pi_{0} E_{n}=\mathbb{W F}_{p^{n}} \llbracket u_{1}, \ldots, u_{n-1} \rrbracket .
$$

The Hopkins-Miller theorem shows that this spectrum admits an action by the group of $G$ automorphisms of $\left(\mathbb{F}_{p^{n}}, F_{n}\right)$ "on the nose". This automorphism group is an extension of the Morava stabilizer group $S_{n}$ by the Galois group of $\mathbb{F}_{p^{n}}$ over $\mathbb{F}_{p}$.

One application of the Hopkins-Miller theorem is the construction of "higher real $K$-theories", which are produced by taking the homotopy fixed points of $E_{n}$ by the action of a finite subgroup of $G$. The fixed points of $E_{n}$ by a maximal finite subgroup of $G$ are usually denoted $E O_{n}$.

We will return to Theorem 2.1 in Section 7 .

\section{Part 1. Universal deformations}

In the following sections, we define universal deformations and describe the Lubin-Tate moduli space. We use this to construct a functor from an appropriate category of formal group laws to the category of generalized homology theories (Section 6). It is this functor which the Hopkins-Miller theorem "lifts" to the category of $\mathcal{A}_{\infty}$-ring spectra.

\section{Formal group laws}

We define the category of formal group laws, and discuss some basic properties. More details on formal group laws may be found in [7], [1, part 2], and [13], among other places. 
3.1. Definition of formal group laws. Let $R$ be a commutative ring. A formal group law $F$ over $R$ is a formal power series $F(x, y) \in R \llbracket x, y \rrbracket$ such that

(a) $F(x, 0)=x=F(0, x)$,

(b) $F(x, y)=F(y, x)$,

(c) $F(F(x, y), z)=F(x, F(y, z))$.

By (a) and (b) such a power series $F(x, y)$ necessarily has the form

$$
F(x, y)=x+y+\sum_{i, j \geq 1} a_{i j} x^{i} y^{j},
$$

where $a_{i j}=a_{j i} \in R$. Sometimes I'll write $\underset{F}{+} y$ for $F(x, y)$.

ExAmple 3.2. The additive formal group law is defined by $x_{G_{a}}^{+} y=x+y$.

EXAmple 3.3. The multiplicative formal group law is defined by $1+(x+y)=$ $(1+x)(1+y)$.

EXAmPle 3.4. Elliptic formal group laws. (See, e.g., Silverman [17].)

Let $B$ is a complete local $R$-algebra with maximal ideal $\mathfrak{m}$; that is, $B \simeq$ $\lim B / \mathfrak{m}^{k}$ and $\bigcap_{k} \mathfrak{m}^{k}=0$. A formal group law $F$ on $R$ defines an abelian group structure on $\mathfrak{m}$. In particular, it defines an abelian group structure on the ideal of $R \llbracket x \rrbracket$ generated by $x$. We write $a+b$ for the sum of $a, b \in \mathfrak{m}$ in this group structure.

A morphism $f: F \rightarrow G$ of formal group laws over $R$ is a formal power series $f(x) \in R \llbracket x \rrbracket$ such that $f(0)=0$ and $f(x+\underset{F}{+} y)=f(x) \underset{G}{+} f(y)$. The isomorphisms are precisely those $f(x) \in R \llbracket x \rrbracket$ for which $f(x)=a x+\cdots$ with $a$ an invertible element of $R$. Such an isomorphism is called a strict isomorphism if $a=1$.

Given a formal group law $F$ over $R$ and a power series $f(x) \in R \llbracket x \rrbracket$ with $f(0)=0$ and with $f^{\prime}(0)$ a unit in $R$, we can define a new formal group law $G$ by

$$
G(x, y)=f\left(F\left(f^{-1}(x), f^{-1}(y)\right)\right),
$$

which comes with an isomorphism $f: F \rightarrow G$ of formal group laws.

I'll write $\operatorname{fgl}(R)$ for the groupoid consisting of all formal group laws on $R$ and all isomorphisms between them. A map $\phi: R \rightarrow S$ of rings induces a functor $\phi^{*}: \operatorname{fgl}(R) \rightarrow \operatorname{fgl}(S)$ by sending

$$
F(x, y)=\sum_{i, j} a_{i j} x^{i} y^{j} \quad \text { to } \quad \phi^{*} F(x, y)=\sum_{i, j} \phi\left(a_{i j}\right) x^{i} y^{j} .
$$

For any formal group law, one can write the $n$-series

$$
[n]_{F}(x)=x+\cdots+\underset{F}{+} x,
$$

by taking the $n$-fold sum of $x$ in the group law $F$. The $n$-series is in fact an endomorphism of the formal group law $F$. Since $[n]_{F}(x)=n x+\cdots$, it is an automorphism if $n$ is invertible in $R$.

3.5. Formal group laws over fields of characteristic $p$. We now consider formal group laws over a field $k$ of characteristic $p$.

Proposition 3.6. If $f: F \rightarrow G$ is a homomorphism of formal group laws over $k$, and if $f(x) \neq 0$, then

$$
f(x)=g\left(x^{p^{n}}\right)
$$


for some $n \geq 0$ and some $g(x) \in k \llbracket x \rrbracket$ with $g(0)=0$ and $g^{\prime}(0) \neq 0$.

For such a homomorphism $f: F \rightarrow G$ the largest such integer $n$ is called the height of $f$ (or if $f(x)=0$, we say $f$ has infinite height.) If $[p]_{F}(x)$ has height $n$ then we say that the formal group law $F$ has height $n$.

Proof of Proposition 3.6. Since $f(F(x, y))=G(f(x), f(y))$, by applying $\frac{\partial}{\partial y}$ and evaluating at $y=0$ we see that

$$
f^{\prime}(x) F_{2}(x, 0)=G_{2}(f(x), 0) f^{\prime}(0) .
$$

Since $F_{2}(x, 0)=1+$ (higher terms), it has a multiplicative inverse, so we see that if $f^{\prime}(0)=0$ then $f^{\prime}(x)=0$, whence $f(x)=g\left(x^{p}\right)$ for some power series $g(x)$. The proof can be completed by induction if we can show that $g(x)$ is itself a morphism of formal group laws.

Let $\sigma: k \rightarrow k$ denote the homomorphism $x \mapsto x^{p}$. Then one can check that

$$
F \stackrel{x^{p}}{\longrightarrow} \sigma^{*} F \stackrel{g}{\rightarrow} G
$$

is a diagram of formal group laws and homomorphisms. In fact,

$$
g\left(\sigma^{*} F\left(x^{p}, y^{p}\right)\right)=g\left(F(x, y)^{p}\right)=f(F(x, y))=G(f(x), f(y))=G\left(g\left(x^{p}\right), g\left(y^{p}\right)\right),
$$

whence

as desired.

$$
g\left(\sigma^{*} F(x, y)\right)=G(g(x), g(y))
$$

EXAmPle 3.7. The additive formal group law has infinite height, since the $p$ series is $[p]_{G_{a}}(x)=p x \equiv 0$. The multiplicative formal group law has height 1 , since $[p]_{G_{m}}(x)=(1+x)^{p}-1 \equiv 1+x^{p}-1=x^{p}$. An elliptic formal group law has height 1 or height 2. (Silverman $[\mathbf{1 7}$, Ch. 7].)

EXAMPLE 3.8. Over $k=\mathbb{F}_{p}$ there is a ( $p$-typical) formal group law $F_{n}$ (the Honda formal group law) with $[p]_{F_{n}}(x)=x^{p^{n}}$. (Ravenel [13, App. 2].)

\section{The universal deformations}

We define deformations and the deformation category, and introduce the universal deformations.

4.1. Deformations of a formal group law. Let $(k, \Gamma)$ be a pair consisting of a field $k$ of characteristic $p>0$ and a formal group $\Gamma$ over $k$. A deformation of $(k, \Gamma)$ to a complete local ring $B$ (with maximal ideal $\mathfrak{m}=\mathfrak{m}_{B}$ and projection $\pi: B \rightarrow B / \mathfrak{m})$ is a pair $(G, i)$ consisting of a formal group law $G$ over $B$ and a homomorphism $i: k \rightarrow B / \mathfrak{m}_{B}$, such that $i^{*} \Gamma=\pi^{*} G$.

A morphism of deformations $\left(G_{1}, i_{1}\right) \rightarrow\left(G_{2}, i_{2}\right)$ is defined only when $i_{1}=i_{2}$, in which case it consists of an isomorphism $f: G_{1} \rightarrow G_{2}$ of formal group laws over $B$ such that $\pi^{*} f$ is the identity map of $\pi^{*} G_{1}=\pi^{*} G_{2}=i_{1}^{*} \Gamma=i_{2}^{*} \Gamma$. That is,

$$
f(x) \equiv x \quad \bmod \mathfrak{m}_{B} .
$$

Such an isomorphism $f$ is sometimes called a $\star$-isomorphism. 
The category of deformations of $\Gamma$ over $B$ is denoted $\operatorname{Def}_{\Gamma}(B)$. It's nice to think of this category using the following picture.

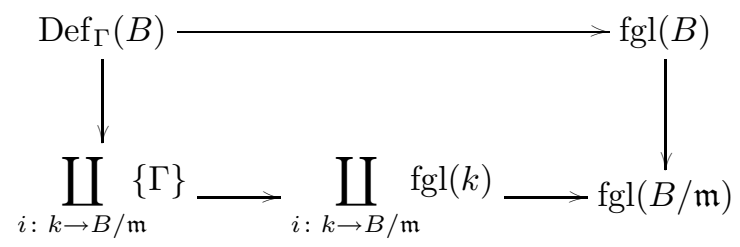

This is a diagram of groupoids. The lower left-hand corner denotes the discrete groupoid, having as objects the set of maps $i: k \rightarrow B / \mathfrak{m}$, and as maps only identity maps. This groupoid sits inside a coproduct of copies of the groupoid $\operatorname{fgl}(k)$; the inclusion maps the "object" $i$ to the pair $(\Gamma, i)$, where $G$ is a formal group law over $k$ and $i: k \rightarrow B / \mathfrak{m}$ a homomorphism. The lower right-hand functor maps such objects to $\operatorname{fgl}(B / \mathfrak{m})$ by sending $(G, i)$ to $i^{*} G$. The diagram commutes, and is a pull-back square of groupoids.

Remark 4.2. Given a morphism $f: C \rightarrow D$ of groupoids, the simplicial nerve of $f$ is a Kan fibration of simplicial sets if and only if $f$ has the following property: for each object $c \in \mathrm{ob} C$ and morphism $\beta: f c \rightarrow d$ in $D$ there exists an $\alpha: c \rightarrow d^{\prime}$ in $C$ such that $f \alpha=\beta$.

Thus, if I apply the classifying space functor to the above diagram, the square remains a pull-back square; furthermore, the right-hand vertical arrow becomes a fibration. Thus, the diagram induces a homotopy pull-back square of classifying spaces.

4.3. The Lubin-Tate theorem. Given $j: k \rightarrow B / \mathfrak{m}$, let $\operatorname{Def}_{\Gamma}(B)_{j}$ denote the full subgroupoid of $\operatorname{Def}_{\Gamma}(B)$ consisting of those deformations $(G, i)$ for which $i=j$.

I will always feel free to replace a groupoid by its classifying space, and thus will speak of $\pi_{0}$ and $\pi_{1}$ of a groupoid, meaning the corresponding homotopy groups of its classifying space. Of course, if $C$ is a groupoid then $\pi_{0} C$ is just the set of isomorphism classes in $C$, and $\pi_{1}(C, X)$ (the fundamental group based at an object $X)$ is the group of automorphisms of the object $X$ in $C$.

Theorem 4.4 (Lubin-Tate). [10] If $\Gamma$ is a formal group law of height $n<\infty$ over $k$, and if $D=(G, i)$ is some deformation of $(k, \Gamma)$, then

$$
\pi_{1}\left(\operatorname{Def}_{\Gamma}(B)_{i}, D\right)=\{1\},
$$

and there is a bijection

$$
\pi_{0}\left(\operatorname{Def}_{\Gamma}(B)_{i}\right) \simeq \mathfrak{m}^{\times(n-1)} .
$$

Furthermore, there exists a complete local ring $E(k, \Gamma)$, an isomorphism $i: k \stackrel{\sim}{\longrightarrow}$ $E(k, \Gamma) / \mathfrak{m}$, and a formal group law $F$ on $E(k, \Gamma)$ such that the pair $(F$, id) is a universal deformation, in the sense that the functor $B \mapsto \pi_{0} \operatorname{Def}_{\Gamma}(B)$ is corepresented by the ring $E(k, \Gamma)$, so that a map $\phi: E(k, \Gamma) \rightarrow B$ corresponds to the isomorphism class of $\phi^{*} F$ in $\operatorname{Def}_{\Gamma}(B)$.

An outline of the proof of Theorem 4.4 is given in Section 5 .

Let me make clear what it means for $(F$, id) to be a universal deformation. Given a deformation $(G, i)$ of $(k, \Gamma)$ to $B$, we have the following diagrams of rings 
and formal group laws.
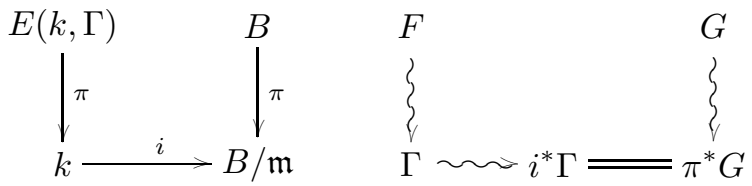

(The squiggly arrows are meant to represent the action of a functor on an object.) For $(F$, id $)$ to be a universal deformation, there must exist a unique map $\phi: E(k, \Gamma) \rightarrow B$ such that the object $\left(\phi^{*} F, i\right)$ is isomorphic to $(G, i)$ in the category $\operatorname{Def}_{\Gamma}(B)$. Then there exists a (necessarily unique) $\star$-isomorphism $g: G \rightarrow \phi^{*} F$. In other words, you get a unique pair of diagrams
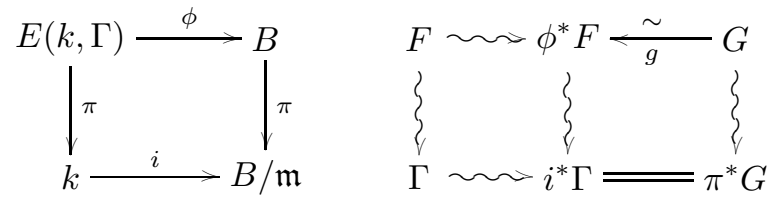

where $g(x) \equiv x \bmod \mathfrak{m}_{B}$.

The first part of Theorem 4.4 says that $\operatorname{fgl}(B) \rightarrow \operatorname{fgl}(B / \mathfrak{m})$ is a "covering space up to homotopy". Thus, an element in $\pi_{1}$ of the base "space" $f g l(B / \mathfrak{m})$ induces an action on $\pi_{0}$ of the "fiber" $\operatorname{Def}_{\Gamma}(B)_{i}$. In particular, given $\gamma \in \pi_{1}(\operatorname{fgl}(k), \Gamma) \simeq$ $\operatorname{Aut}(\Gamma)$, we obtain an induced map $\tilde{\gamma}: \pi_{0} \operatorname{Def}_{\Gamma}(B)_{i} \rightarrow \pi_{0} \operatorname{Def}_{\Gamma}(B)_{i}$ for each $i: k \rightarrow$ $B / \mathfrak{m}$. Putting these together over all the $i$ 's we get a map $\tilde{\gamma}: \pi_{0} \operatorname{Def}_{\Gamma}(B) \rightarrow$ $\pi_{0} \operatorname{Def}_{\Gamma}(B)$. It's easy to see that this action is natural in $B$; in other words, there is an induced action of $\operatorname{Aut}(\Gamma)$ on the functor $\pi_{0} \operatorname{Def}_{\Gamma}(-)$, and hence on the ring $E(k, \Gamma)$ which represents the functor.

If $k=\mathbb{F}_{p^{n}}$, and $\Gamma=F_{n}$ is the Honda formal group law of height $n$, then $\operatorname{Aut}\left(F_{n}\right)$ contains the $n$-th Morava stabilizer group $S_{n}$ as the group of strict automorphisms of $F_{n}$.

We can sometimes get a bigger group action on $E(k, \Gamma)$. Suppose $k$ is a finite field, and fix a map $i_{0}: k \rightarrow B / \mathfrak{m}$. Then maps $i: k \rightarrow B / \mathfrak{m}$ can be factored $i=i_{0} \sigma^{d}$, where $\sigma: k \rightarrow k$ denotes $\sigma(x)=x^{p}$. If $\Gamma$ is Galois invariant, i.e., if $\sigma^{*} \Gamma=\Gamma$ (for example, if $\Gamma$ is the Honda formal group law), then there is an action of $\operatorname{Gal}\left(\mathbb{F}_{p^{n}} / \mathbb{F}_{p}\right)=C_{n}$ on $\operatorname{Def}_{\Gamma}(B)$, and hence on $E(k, \Gamma)$.

4.5. The ring $E(k, \Gamma)$. We can say something about the $\operatorname{ring} E(k, \Gamma)$, at least if $k$ is a perfect field. Since it is a complete local ring with residue field $k$, it must contain the Witt vectors $\mathbb{W} k$. For $k=\mathbb{F}_{p^{n}}, \mathbb{W} k=\mathbb{Z}_{p}[\zeta]$, where $\zeta$ is a primitive $\left(p^{n}-1\right)$ st root of unity. For a perfect field $k$ the Witt ring $\mathbb{W} k$ has the following universal property; given a diagram

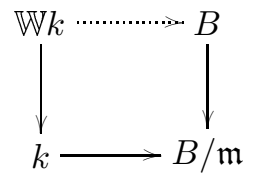

where $B$ is a complete local ring and all maps are continuous homomorphisms, there is a unique dotted arrow making the diagram commute. (This is essentially [16, II.5].) This is easy to see when $k=\mathbb{F}_{p^{n}}$, since in this case the Teichmüller construction ensures that one can produce a canonical multiplicative section $k \rightarrow B$. 
Thus $E(k, \Gamma)$ is a $\mathbb{W} k$-algebra, and in fact contains $\mathbb{W} k$ (because there exists at least one deformation of $\Gamma$ to $\mathbb{W} k$, so that there exists a map from $E(k, \Gamma)$ back down to $\mathbb{W} k$.) Since a map $i: k \rightarrow B / \mathfrak{m}$ determines a unique map $\mathbb{W} k \rightarrow B$, making $B$ also into a $\mathbb{W} k$-algebra, then the isomorphism of Theorem 4.4

$$
\operatorname{Hom}_{\mathbb{W} k-\operatorname{alg}}(E(k, \Gamma), B) \simeq \pi_{0} \operatorname{Def}_{\Gamma}(B)_{i} \simeq \mathfrak{m}^{\times(n-1)}
$$

indicates that we should set

$$
E(k, \Gamma) \simeq \mathbb{W} \mathbb{F}_{p^{n}} \llbracket u_{1}, \ldots, u_{n-1} \rrbracket .
$$

4.6. Functoriality of the universal deformation. Consider a morphism $\alpha:\left(k_{1}, \Gamma_{1}\right) \rightarrow\left(k_{2}, \Gamma_{2}\right)$ in the category $\mathcal{F G} ; \alpha$ is a pair $(j, f)$ with $j: k_{2} \rightarrow k_{1}$ and $f: \Gamma_{1} \stackrel{\sim}{\longrightarrow} j^{*} \Gamma_{2}$. Such a morphism induces a diagram

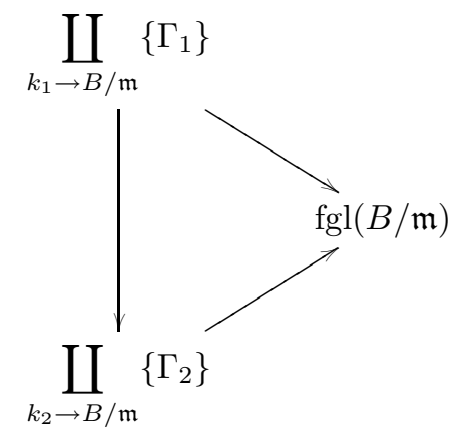

which commutes up to a homotopy (determined by $f$ ). This induces a map

$$
\pi_{0} \operatorname{Def}_{\Gamma_{1}}(B) \rightarrow \pi_{0} \operatorname{Def}_{\Gamma_{2}}(B)
$$

which is natural in $B$, and hence a map $E\left(k_{2}, \Gamma_{2}\right) \rightarrow E\left(k_{1}, \Gamma_{1}\right)$. Thus we obtain, according to Theorem 4.4, a functor

$$
\mathcal{F} \mathcal{G}^{\mathrm{op}} \rightarrow\{\text { complete local rings }\}
$$

sending

$$
(k, \Gamma) \mapsto E(k, \Gamma) .
$$

\section{Construction of the universal deformation}

I give a construction of the universal deformation, and at least indicate how the proof goes. I mainly follow Lubin-Tate's original proof [10], though I also borrow an observation of Drinfel'd [4].

5.1. Deformations to a trivial square 0 extension over $k$. I want to give at least an idea of how the proof goes. So what I'll do first is compute $\operatorname{Def}_{\Gamma}\left(k[\epsilon] / \epsilon^{2}\right)$.

Let $R=k[\epsilon] / \epsilon^{2}$. The inclusion $k \rightarrow R$ allows us to push a formal group law $\Gamma$ on $k$ to $R$; by abuse of notation, I will also refer to the pushed-forward-to- $R$ formal group law as $\Gamma$. An arbitrary formal group law on $R$ which lifts $\Gamma$ must look something like

$$
F(x, y)=\underset{\Gamma}{+} \underset{\Gamma}{+} \epsilon G(x, y)
$$

where $G(x, y) \in k \llbracket x, y \rrbracket$. For $F$ to really be a formal group law, we must also have

(a) $G(x, 0)=0=G(0, y)$,

(b) $G(x, y)=G(y, x)$, 
(c) $G(\underset{\Gamma}{x}+y, z)+G(x, y)=G(x, y \underset{\Gamma}{+} z)+G(y, z)$.

Such a $G$ is called a $\Gamma$-symmetric 2 -cocycle. I'll write $Z_{\mathrm{sym}}^{2}(\Gamma)$ for the set of such cocycles.

Now suppose I have another formal group law $F^{\prime}(x, y)=\underset{\Gamma}{x} \underset{\Gamma}{\sin }+\epsilon G^{\prime}(x, y)$, and a map $f: F \rightarrow F^{\prime}$ which reduces to the identity map $\bmod \epsilon$. Such an $f$ has the form,

$$
f(x)=\underset{\Gamma}{x}+\epsilon g(x)
$$

for some $g(x) \in k \llbracket x \rrbracket$ with $g(0)=0$. We shall call such a $g$ a $\Gamma$-symmetric 1-cocycle, and write $Z_{\mathrm{sym}}^{1}(\Gamma)$ for the set of all such cocycles.

Then $f(x+\underset{F}{+} y)=f(x) \underset{F^{\prime}}{+} f(y)$ if and only if

$$
G(x, y)+g(\underset{\Gamma}{x}+y)=G^{\prime}(x, y)+g(x)+g(y) .
$$

This means that we can form a little chain complex:

$$
0 \longrightarrow\left\{\begin{array}{c}
g(x) \in k \llbracket x \rrbracket, \\
g(0)=0
\end{array}\right\} \stackrel{\delta}{\longrightarrow}\left\{\begin{array}{c}
G(x, y) \in k \llbracket x, y \rrbracket \\
G \text { a } \Gamma \text {-symmetric 2-cocycle }
\end{array}\right\} \longrightarrow 0
$$

where $\delta g(x, y)=g(x)+g(y)-g(x+\underset{\Gamma}{+} y)$. The homology of this chain complex is precisely the homotopy of $\operatorname{Def}_{\Gamma}(R)_{\text {id }}$. That is, the cokernel of $\delta$ may be identified with the set of isomorphism classes in $\operatorname{Def}_{\Gamma}(R)_{\text {id }}$, and the kernel can be identified as the group of automorphisms of any chosen object of $\operatorname{Def}_{\Gamma}(R)_{\mathrm{id}}$.

5.2. Lemmas about formal group laws. To compute this complex, we need a couple of lemmas. First, note that an additive symmetric cocycle is a symmetric cocycle for the additive group.

Lemma 5.3. An additive symmetric cocycle $G(x, y)$, homogeneous of degree d, has the form $a C_{d}(x, y)$ for some $a \in k$, and where $C_{d}(x, y) \in \mathbb{Z} \llbracket x, y \rrbracket$ is defined by

$$
C_{d}(x, y)=\frac{1}{\lambda_{d}}\left(x^{d}+y^{d}-(x+y)^{d}\right)
$$

with

$$
\lambda_{d}=\left\{\begin{array}{ll}
1 & \text { if } d \neq p^{j} \\
p & \text { if } d=p^{j}
\end{array} .\right.
$$

Proof. See Fröhlich [7]. The proof is also given in [1, part 2].

Lemma 5.4. Let $\pi: R \rightarrow S$ be a surjective map of rings. If $F(x, y) \in R \llbracket x, y \rrbracket$ satisfies the axioms for a formal group law modulo terms of degree $(d+1)$, and if $\pi^{*} F$ is a formal group law over $S$, then there exists a formal group law $F^{\prime}(x, y) \in R \llbracket x, y \rrbracket$ such that $F^{\prime} \equiv F \bmod$ degree $(d+1)$ terms and such that $\pi^{*} F^{\prime}=\pi^{*} F$.

Lemma 5.4 really follows from the theorem of Lazard.

Theorem 5.5 (Lazard). There exists a ring $L \simeq \mathbb{Z}\left[s_{1}, s_{2}, \ldots\right]$ and a formal group law $F$ over $L$ such that the induced map

$$
\operatorname{Hom}_{\text {Ring }}(L, R) \rightarrow \operatorname{obfgl}(R)
$$

sending $f \mapsto f^{*} F$ is a bijection. Furthermore, if we write

$$
x+\underset{F}{x} y=x+y+\sum_{i, j} a_{i j} x^{i} y^{j}
$$


then $a_{i j} \in L$ is a polynomial in the variables $s_{1}, \ldots, s_{i+j-1}$.

Proof. See [7], [1, part 2] or [13, Appendix 2].

Lemma 5.3 shows that any $\Gamma$-symmetric 2-cocycle has the form

$$
G_{d}(x, y)=a C_{d}(x, y)+\text { higher degree terms, }
$$

since the lowest degree term of a $\Gamma$-symmetric 2-cocycle must necessarily be an additive cocycle. Lemma 5.4 shows that such a $\Gamma$-symmetric 2 -cocycle exists, since the expression

$$
F(x, y)=\underset{\Gamma}{x} \underset{\Gamma}{+} \underset{\Gamma}{t} C_{d}(x, y)
$$

is a formal group law modulo degree $(d+1)$, and modulo $\mathfrak{m}$ is the formal group law $\Gamma$.

There is an obvious filtration $\cdots \supset F_{d} Z_{\text {sym }}^{2}(\Gamma) \supset F_{d+1} Z_{\text {sym }}^{2}(\Gamma) \supset \cdots$ of the symmetric 2-cocycles in which $F_{d} Z_{\text {sym }}^{2}(\Gamma)$ consists of all elements whose lowest degree term is at least $d$. Thus we have shown that the associated quotients of this complete filtration $F_{d} Z_{\text {sym }}^{2} / F_{d+1} Z_{\text {sym }}^{2}$ are each isomorphic to $k$, generated by an element $G_{d}$.

Similarly, there is a filtration $\cdots \supset F_{d} Z_{\text {sym }}^{1}(\Gamma) \supset F_{d+1} Z_{\text {sym }}^{1}(\Gamma) \supset \cdots$ where $F_{d} Z_{\text {sym }}^{1}(\Gamma)$ consists of all elements whose lowest degree term is at least $d$. Thus each associated quotient $F_{d} Z_{\text {sym }}^{1} / F_{d+1} Z_{\text {sym }}^{1}$ is isomorphic to $k$, generated by an element $f_{d}(x)=x^{d}$. Thus $Z_{\text {sym }}^{\bullet}$ is a filtered chain complex, and all we need to do is calculate the associated spectral sequence!

Let's assume that

$$
\Gamma(x, y)=x+y+b C_{p^{n}}(x, y)+\cdots .
$$

We can do this without loss of generality, by the following lemma, and by the fact that isomorphic formal group laws have isomorphic complexes $Z_{\text {sym }}^{\bullet}$ of symmetric cocycles.

LEMMA 5.6. A height $n$ formal group law $\Gamma$ over $k$ is isomorphic to a formal group law $\Gamma^{\prime}$ having the form

$$
\Gamma^{\prime}(x, y)=x+y+b C_{p^{n}}(x, y)+\cdots,
$$

with $b \neq 0$.

Proof. This is because if $\Gamma$ has the form

$$
\Gamma(x, y) \equiv x+y+b C_{k}(x, y)+(x, y)^{k+1}
$$

for $k \neq p^{j}$, we can let $f(x)=x+b x^{k}$ and $\Gamma^{\prime}(x, y)=f\left(\Gamma\left(f^{-1} x, f^{-1} y\right)\right)$, whence $f: \Gamma \rightarrow \Gamma^{\prime}$ is an isomorphism of formal group laws, and

$$
\Gamma^{\prime}(x, y) \equiv x+y+(x, y)^{k+1} .
$$

If $\Gamma$ has the form

$$
\Gamma(x, y)=x+y+b C_{p^{j}}(x, y)+(x, y)^{p^{j}+1}
$$

one can show by Lemma 5.7 that $[p]_{\Gamma}(x)=b x^{p^{j}}+\cdots$, hence $b=0$ or $j=n$. 
LEMMA 5.7. If $F$ is a formal group law with

$$
x+\underset{F}{+} y=x+y+b C_{k}(x, y)+\cdots
$$

then

$$
[n]_{F}(x)=n x+b\left\{\frac{1}{\lambda_{k}}\left(n-n^{k}\right)\right\} x^{k}+\cdots .
$$

5.8. Computation of the chain complex. Let $f_{k}(x)=x^{k}$. Then

$$
\begin{aligned}
\left(\delta f_{k}\right)(x, y) & =x^{k}+y^{k}-(x+y)^{k} \\
& = \begin{cases}C_{k}(x, y)+\cdots & \text { if } k \neq p^{j}, \text { where } j \geq 0, \\
b^{p^{j}} C_{p^{n}}(x, y)^{p^{j}}+\cdots & \text { if } k=p^{j}, \text { where } j \geq 0 .\end{cases}
\end{aligned}
$$

Since $C_{p^{n}}(x, y)=C_{p}(x, y)^{p^{n-1}}$, we see that

$$
\delta f_{k}= \begin{cases}G_{k} \bmod F_{k+1} Z_{\mathrm{sym}}^{2} & \text { if } k \neq p^{j} \\ G_{p^{n+j}} \bmod F_{p^{n+j}+1} Z_{\mathrm{sym}}^{2} & \text { if } k=p^{j}\end{cases}
$$

Thus the cokernel of $\delta$ has dimension $(n-1)$ over $k$, generated by elements of the form

$$
G_{p^{k}}^{\prime}(x, y)=C_{p^{k}}(x, y)+\cdots
$$

for $k=1, \ldots, n-1$. The kernel of $\delta$ is trivial. Hence

$$
\pi_{0} \operatorname{Def}_{\Gamma}(R)_{i}=H_{\mathrm{sym}}^{2}(\Gamma) \simeq k^{\times(n-1)},
$$

and

$$
\pi_{1} \operatorname{Def}_{\Gamma}(R) \simeq 0
$$

5.9. The case of a general square-zero extension. The above argument proves the first part of the Lubin-Tate theorem for $B=k[\epsilon] / \epsilon^{2}$. The method of proof extends to prove a more general statement.

Let $B$ be a complete local ring $B$ with maximal ideal $\mathfrak{m}$ and residue field $k=B / \mathfrak{m}$. Let $F_{k}$ be a formal group law over $B / \mathfrak{m}^{k}$ and let $\Gamma$ denote its pushforward to $k$. Suppose $F_{k+1}$ is a formal group law over $B / \mathfrak{m}^{k+1}$ which lifts $F_{k}$. Then any other formal group law $F_{k+1}^{\prime}$ over $B / \mathfrak{m}^{k+1}$ which lifts $F_{k}$ has the form

$$
F_{k+1}^{\prime}(x, y)=F_{k}(x, y) \underset{F_{k+1}}{+} G(x, y)
$$

where $G(x, y) \in Z_{\text {sym }}^{2}(\Gamma) \hat{\otimes} \mathfrak{m}^{k} / \mathfrak{m}^{k+1}$. Furthermore, an isomorphism $f: F_{k+1} \rightarrow$ $F_{k+1}^{\prime}$ which lifts the identity map of $F_{k}$ has the form

$$
f(x)=x \underset{F_{k+1}}{+} g(x)
$$

where $g(x) \in Z_{\text {sym }}^{1}(\Gamma) \hat{\otimes} \mathfrak{m}^{k} / \mathfrak{m}^{k+1}$.

Thus the "fiber" of $\operatorname{fgl}\left(B / \mathfrak{m}^{k+1}\right) \rightarrow \operatorname{fgl}\left(B / \mathfrak{m}^{k}\right)$ over $F_{k}$ has $H_{\text {sym }}^{2}(\Gamma) \otimes \mathfrak{m}^{k} / \mathfrak{m}^{k+1}$ as its set of components, and $H_{\text {sym }}^{1}(\Gamma) \otimes \mathfrak{m}^{k} / \mathfrak{m}^{k+1}$ as the set of isomorphisms of any object.

The above can be proved most easily by pulling back the map $\pi: B / \mathfrak{m}^{k+1} \rightarrow$ $B / \mathfrak{m}^{k}$ along itself; the pulled-back map is a trivial square-0 extension, and the argument of the previous sections can be applied to it. 
5.10. Construction of the universal deformation. To finish the proof, we need to construct the universal deformation. This will be a formal group law over the ring

$$
E(k, \Gamma)=\mathbb{W} k \llbracket u_{1}, \ldots, u_{n-1} \rrbracket .
$$

It turns out that it suffices to produce a formal group law over this ring which has the form

$$
F(x, y) \equiv \underset{\Gamma}{x} \underset{\Gamma}{+}+u_{1} C_{p}(x, y) \underset{\Gamma}{+}+\cdots+\underset{\Gamma}{+} u_{n-1} C_{p^{n-1}}(x, y) \underset{\Gamma}{+}+\cdots
$$

modulo $p$ and modulo $\left(u_{1}, \ldots, u_{n-1}\right)^{2}$. It is easy to construct such: we can define a formal group law over $k \llbracket u_{1}, \ldots, u_{n-1} \rrbracket /\left(u_{1}, \ldots, u_{n-1}\right)^{2}$ by

$$
x \underset{F}{+} y=\underset{\Gamma}{+} \underset{\Gamma}{+} u_{1} G_{p}(x, y) \underset{\Gamma}{+}+\underset{\Gamma}{+} u_{n-1} G_{p^{n-1}}(x, y),
$$

where $G_{p^{j}}(x, y) \in Z_{\mathrm{sym}}^{2}(\Gamma)$ are the symmetric cocycles we constructed above. This group law then lifts up to $\mathbb{W} k \llbracket u_{1}, \ldots, u_{n-1} \rrbracket$, by Lazard's Theorem 5.5.

The proof is completed by considering the map

$$
\operatorname{Hom}_{\mathbb{W} k-\operatorname{alg}}(E(k, \Gamma), B) \rightarrow \pi_{0} \operatorname{Def}_{\Gamma}(B)_{i}
$$

which sends $f$ to the isomorphism class of $f^{*} F$, where $F$ is the above constructed formal group law. The left hand side is isomorphic as a set to $\mathfrak{m}^{\times(n-1)}$, which has a natural filtration by powers of $\mathfrak{m}$. The right hand side also has a filtration via the kernels of the maps $\pi_{0} \operatorname{Def}_{\Gamma}(B)_{i} \rightarrow \pi_{0} \operatorname{Def}_{\Gamma}\left(B / \mathfrak{m}^{k}\right)_{i}$, and one shows the map is an isomorphism by comparing filtration quotients; of course, if $B=k[\epsilon] / \epsilon^{2}$, then it is clearly an isomorphism by what we have shown.

EXAMPLE 5.11. Let $n=1$ and $k=\mathbb{F}_{p}$, and consider the multiplicative formal group law $G_{m}(x, y)=x+y+x y$ over $\mathbb{F}_{p}$. Then

$$
E\left(\mathbb{F}_{p}, G_{m}\right) \simeq \mathbb{Z}_{p}
$$

and we can take as the universal deformation $F(x, y)=x+y+x y$.

\section{The homology theories $E_{k, \Gamma}$}

In this section we use universal deformation theory to construct homology theory $E_{k, \Gamma}$ for each formal group law $(k, \Gamma)$. In fact, we construct a functor $E$ from the category $\mathcal{F G}$ of formal group laws to the category of homology theories.

6.1. Complex orientations and formal group laws. A reference for material in this section is from [1, part 2].

A ring spectrum $E$ is complex orientable if there exists a cohomology class $x \in \tilde{E}^{2} \mathbb{C P}^{\infty}$ such that the restriction of this class to $\mathbb{C P}^{1} \simeq S^{2}$ is the generator of $\tilde{E}^{2} S^{2} \simeq \pi_{0} E$. Such a class determines a characteristic class $c_{1}(L) \in E^{2}(X)$ for a complex line bundle $L$ over $X$.

For a complex oriented cohomology theory, $E^{*} \mathbb{C P}^{\infty} \simeq E^{*} \llbracket x \rrbracket$. Thus the orientation determines a formal group law $F$, induced by the map $\mathbb{C P}^{\infty} \times \mathbb{C P}^{\infty} \rightarrow \mathbb{C P}^{\infty}$ which corresponds to tensor product of line bundles. Thus

$$
c_{1}\left(L \otimes L^{\prime}\right)=c_{1}(L) \underset{F}{+} c_{1}\left(L^{\prime}\right) .
$$

Note that this formal group law is of the form

$$
F(x, y)=x+y+\sum_{i j} a_{i j} x^{i} y^{j}
$$


for $a_{i j} \in \pi_{2(i+j-1)} E$. I'll call this a degree -2 formal group law, since the equation defining $F(x, y)$ is in degree -2 . (That is, it's an element of $\pi_{-2} \mathcal{F}\left(\mathbb{C P}^{\infty}, E\right)$, where $\mathcal{F}(X, Y)$ denotes the function spectrum from $X$ to $Y$.)

For example, the complex cobordism spectrum $M U$ is complex orientable. The two inclusions of $\eta_{L}, \eta_{R}: M U \rightrightarrows M U \wedge M U$ induce two formal group laws over $M U_{*} M U$ and a canonical strict isomorphism between them.

TheOREM 6.2 (Quillen). The canonical formal group law $F$ over $M U_{*}$ is the universal formal group law (of degree -2) in the sense that, given a formal group law $G$ of degree -2 over a graded ring $R_{*}$, there exists a unique map $\phi: M U_{*} \rightarrow R_{*}$ such that $\phi^{*} F=G$.

Furthermore, the canonical isomorphism $f: \eta_{R}^{*} F \rightarrow \eta_{L}^{*} F$ over $M U_{*} M U$ is the universal strict isomorphism in the sense that, given a strict isomorphism $g: G \rightarrow$ $G^{\prime}$ of formal group laws over $R_{*}$, there exists a unique map $\psi: M U_{*} M U \rightarrow R_{*}$ such that $\psi^{*} f=g$.

Sometimes we want to consider a cohomology theory $E$ which is periodic; e.g., which has an element $u \in \pi_{2} E$ which is invertible. Such an element will allow us to switch between degree -2 formal group laws and degree 0 ones (a degree 0 formal group law is one whose coefficients lie in degree 0 ).

Let $R_{*}$ be a graded ring, such that $R_{2}$ contains an invertible element. Given a formal group law $F$ defined over $R_{0}$ and a chosen invertible element $u_{F} \in R_{2}$, we produce a degree -2 formal group law as follows: define

$$
\bar{F}(x, y)=u_{F}^{-1} F\left(u_{F} x, u_{F} y\right) .
$$

Thus if $F(x, y)=\sum a_{i j} x^{i} y^{j}$, then $\bar{F}(x, y)=\sum a_{i j} u_{F}^{i+j-1} x^{i} y^{j}$ is a degree -2 formal group law.

Given a morphism $g: F \rightarrow G$ of formal group laws over $R_{0}$, and chosen elements $u_{F}, u_{G} \in R_{2}$, we of course get corresponding formal group laws $\bar{F}$ and $\bar{G}$ of degree -2 ; let

$$
\bar{g}(x)=u_{G}^{-1} g\left(u_{F} x\right)
$$

Then $\bar{g}: \bar{F} \rightarrow \bar{G}$ is a morphism of degree -2 formal group laws. Note that $\bar{g}^{\prime}(0)=$ $u_{G}^{-1} u_{F} g^{\prime}(0)$.

6.3. The Landweber exact functor theorem. Given $(k, \Gamma) \in \mathcal{F G}$ we define a functor $E_{k, \Gamma}$ on spaces by

$$
\left(E_{k, \Gamma}\right)_{*}(X) \simeq E(k, \Gamma)\left[u^{ \pm}\right] \underset{M U_{*}}{\stackrel{\bar{F}}{\otimes}} M U_{*}(X) .
$$

The " $\bar{F}$ " sitting on top of the tensor product symbol is meant to symbolize the fact that the tensor product is induced by the map $M U_{*} \rightarrow E(k, \Gamma)\left[u^{ \pm}\right]$which classifies $\bar{F}$, which is the degree -2 formal group law induced from $F$ using $u_{F}=u$. That this is a homology theory comes from the Landweber exact functor theorem, as we will show below.

TheOREM 6.4 (Landweber). Let $R$ be a $M U_{*}$ module. Suppose for each prime $p$ and each $n$ the map

$$
R / p, \ldots, v_{n-1} \stackrel{\cdot v_{n}}{\longrightarrow} R / p, \ldots, v_{n-1}
$$


is injective, where $v_{n}$ is the coefficient of $x^{p^{n}}$ in the p-series of the universal formal group law over $M U_{*}$. (That is, $p, v_{1}, v_{2}, \ldots$ is a regular sequence for $R$.) Then the functor

is a homology theory.

$$
X \mapsto R_{*} \underset{M U_{*}}{\otimes} M U_{*}(X)
$$

Proof. Proved in [8].

Proposition 6.5. The map $\bar{F}: M U_{*} \rightarrow E(k, \Gamma)\left[u^{ \pm}\right]$which classifies a degree -2 universal deformation satisfies the hypotheses of the Landweber exact functor theorem.

We will return to the proof of Proposition 6.5 in section 6.7.

REMARK 6.6.

1. If $R_{*}$ is a ring with a degree -2 formal group law $\bar{F}$, we use the classifying map $M U_{*} \rightarrow R_{*}$ to produce the module structure on $R_{*}$.

2. It turns out that if we have two degree -2 formal group laws $\bar{F}$ and $\bar{G}$ over $R_{*}$ which satisfy the Landweber condition, and are strictly isomorphic by a strict isomorphism $\bar{g}$, there is an induced equivalence of homology theories, via a map

$$
R_{*} \underset{M U_{*}}{\stackrel{\bar{F}}{\otimes}} M U_{*}(X) \rightarrow R_{*} \underset{M U_{*}}{\stackrel{\bar{F}}{\otimes}} M U_{*} M U \underset{M U_{*}}{\otimes} M U_{*}(X) \rightarrow R_{*} \underset{M U_{*}}{\stackrel{\bar{G}}{\otimes}} M U_{*}(X)
$$

where the left hand map is induced by the comultiplication

$$
M U_{*} \rightarrow M U_{*} M U \underset{M U_{*}}{\otimes} M U_{*} X,
$$

and the right hand map is induced by the map $M U_{*} M U \rightarrow R_{*}$ which classifies $\bar{g}$.

6.7. Construction of the functor. We define a functor

$$
E: \mathcal{F} \mathcal{G}^{\text {op }} \rightarrow\{\text { homology theories }\},
$$

as follows. For each object $(k, \Gamma)$ in $\mathcal{F G}$, choose a universal deformation $F$ of $(k, \Gamma)$ to the ring $E(k, \Gamma)$, and let $E_{k, \Gamma}$ be the homology theory constructed above using the Landweber theorem. Let $E(k, \Gamma)_{*}=E(k, \Gamma)\left[u_{F}^{ \pm}\right]$denote the corresponding graded ring

Suppose we have a map $\alpha:\left(k_{1}, \Gamma_{1}\right) \rightarrow\left(k_{2}, \Gamma_{2}\right)$ in $\mathcal{F G}$; such an $\alpha$ consists of maps $i: k_{2} \rightarrow k_{1}$ and $f: \Gamma_{1} \rightarrow i^{*} \Gamma_{2}$. Given universal deformations $F_{1}$ of $\left(k_{1}, \Gamma_{1}\right)$ and $F_{2}$ of $\left(k_{2}, \Gamma_{2}\right)$, we get an induced map

$$
\psi: E\left(k_{2}, \Gamma_{2}\right) \rightarrow E\left(k_{1}, \Gamma_{1}\right)
$$

and an isomorphism

$$
g: F_{2} \rightarrow \psi^{*} F_{1}
$$

such that $g \equiv f \bmod \mathfrak{m}$. That is, $(\psi, g)$ is the unique pair of maps fitting into the diagrams
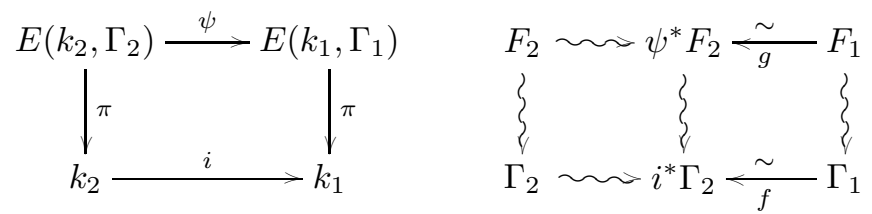
We extend $\psi$ to a map $E\left(k_{2}, \Gamma_{2}\right)\left[u_{F_{2}}^{ \pm}\right] \rightarrow E\left(k_{1}, \Gamma_{1}\right)\left[u_{F_{1}}^{ \pm}\right]$by sending

$$
\psi\left(u_{F_{2}}\right)=g^{\prime}(0) u_{F_{1}} \text {. }
$$

The point here is that we have a diagram

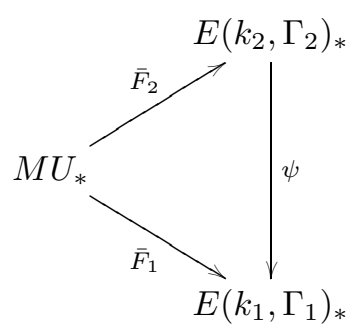

which does not commute, since $\psi^{*} \bar{F}_{2} \neq \bar{F}_{1}$. Instead, we may define $E_{\alpha}: E_{k_{2}, \Gamma_{2}} \rightarrow$ $E_{k_{1}, \Gamma_{1}}$ to be the natural isomorphism of homology theories defined via the composite $E\left(k_{2}, \Gamma_{2}\right)_{*} \underset{M U_{*}}{\stackrel{\bar{F}_{2}}{\otimes}} M U_{*}(X) \rightarrow E\left(k_{1}, \Gamma_{1}\right)_{*} \underset{M U_{*}}{\stackrel{\psi^{*} \bar{F}_{2}}{\otimes}} M U_{*}(X) \stackrel{\sim}{\sim} E\left(k_{1}, \Gamma_{1}\right)_{*} \underset{M U_{*}}{\stackrel{\bar{F}_{1}}{\otimes}} M U_{*}(X)$ where the left hand arrow is induced by $\psi$, and the right hand arrow is induced by the strict isomorphism $\bar{g}: \bar{F}_{1} \rightarrow \psi^{*} \bar{F}_{2}$ defined by

$$
\bar{g}(x)=\left(\psi u_{F_{2}}\right)^{-1} g\left(u_{F_{1}} x\right)=x+\cdots .
$$

It is not too hard to show that $E$ so defined is really a functor.

EXAmple 6.8. Let $(k, \Gamma)=\left(\mathbb{F}_{p}, G_{m}\right)$. Then $\operatorname{Aut}(k, \Gamma) \simeq \mathbb{Z}_{p}^{\times}$, where $n \in \mathbb{Z}_{p}$ corresponds to the map

$$
[n]_{\Gamma}(x)=(1+x)^{n}-1 .
$$

Since this has height one, $E(k, \Gamma) \simeq \mathbb{Z}_{p}$. We can use the multiplicative formal group law as its own universal lift:

$$
x+\underset{F}{x}=x+y+x y .
$$

This induces a degree -2 formal group law on $\mathbb{Z}_{p}\left[u^{ \pm}\right]$given by

$$
x+\frac{\bar{F}}{y}=x+y+u x y .
$$

The morphism $[n]_{\Gamma}(x)$ therefore lifts to an automorphism $[n]_{F}(x)=(1+x)^{n}-1=$ $n x+\cdots$ of $F$, and hence induces an automorphism $[n]_{\bar{F}}(x)=u^{-1}\left((1+u x)^{n}-1\right)$ of $\bar{F}$. We thus see that an automorphism $[n]_{\Gamma}$ of $\Gamma$ lifts to a map $\psi_{n}: \mathbb{Z}_{p}\left[u^{ \pm}\right] \rightarrow \mathbb{Z}_{p}\left[u^{ \pm}\right]$ which sends $\psi_{n}(u)=n u$.

The theory $E_{\mathbb{F}_{p}, G_{m}}$ is precisely complex $K$-theory completed at $p$, and the $\psi_{n}$ 's are precisely the Adams operations.

6.9. Proof of Landweber exactness. It remains to show that the map $M U_{*} \rightarrow E(k, \Gamma)\left[u^{ \pm}\right]$is Landweber exact. It clearly suffices to show that that the sequence $p, w_{1}, w_{2}, \ldots$ is regular in $E(k, \Gamma)$, where

$$
[p]_{F}(x)=p x+\cdots+w_{1} x^{p}+\cdots+w_{2} x^{p^{2}}+\cdots .
$$

(In fact, $w_{i}=v_{i} u^{1-p^{i}}$.) 
The cokernel of multiplication by $p$ is $k \llbracket u_{1}, \ldots, u_{n-1} \rrbracket$. We need to compute the $p$-series of $F$ over this ring. By Lemma 5.6 we can assume without loss of generality that $x+y=x+y+b C_{p^{n}}(x, y)+\cdots$, whence

$$
x+\underset{F}{+} y \equiv x+y+u_{1} G_{p}(x, y)+\cdots+u_{n-1} G_{p^{n-1}}(x, y)+b C_{p^{n}}(x, y)+\cdots
$$

modulo $p$ and $\left(u_{1}, \ldots, u_{n-1}\right)^{2}$ for certain $\Gamma$-symmetric 2-cocycles $G_{i}$. Recall that

$$
G_{p^{i}}(x, y)=C_{p^{i}}(x, y)+\text { higher terms. }
$$

LEMMA 6.10. We have that

$$
[p]_{F}(x)=u_{i} x^{p^{i}}+\cdots
$$

modulo $p, u_{1}, \ldots, u_{i-1}$ and $\left(u_{i}, \ldots, u_{n-1}\right)^{2}$, and

$$
[p]_{F}(x)=b x^{p^{n}}+\cdots
$$

modulo $p, u_{1}, \ldots, u_{n-1}$.

Proof. This is an immediate consequence of Lemma 5.7.

Lemma 6.10 implies that the inclusion

$$
k \llbracket w_{1}, \ldots, w_{n-1} \rrbracket \rightarrow k \llbracket u_{1}, \ldots, u_{n-1} \rrbracket
$$

is in fact an isomorphism (since the Jacobian matrix $\left(\frac{\partial w_{i}}{\partial u_{j}}\right)$ modulo $\left(u_{1}, \ldots, u_{n-1}\right)^{2}$ is upper triangular with ones on the diagonal by Lemma 6.10.) Clearly, the sequence $w_{1}, w_{2}, \ldots$ is regular in this ring.

\section{The construction}

In the next few sections I will discuss the category of spectra (after LewisMay-Steinberger), and categories of $\mathcal{A}_{\infty}$-ring spectra, denoted $\mathcal{A}_{\infty}$. The first thing to note is that $\mathcal{A}_{\infty}$ is a topological category; i.e., for any two objects, there is a topological space of maps. (See Section 12.)

Let $\mathcal{A}_{\infty}^{\mathrm{LT}} \subset \mathcal{A}_{\infty}$ denote the full topological subcategory consisting of $\mathcal{A}_{\infty}$-ring spectra $E$ such that

1. $E$ is cofibrant as an $\mathcal{A}_{\infty}$ ring, (see Section 12),

2. the underlying spectrum of $E$ is a homotopy commutative ring spectrum, which

3. has $\pi_{\text {odd }} E=0$,

4. has an invertible element in $\pi_{2} E$, and such that

5. $\pi_{0} E$ is a complete local ring, with maximal ideal $\mathfrak{m}$, such that

6. $\pi_{0} E / \mathfrak{m}$ is a perfect field of non-zero characteristic, for which

7. the degree 0 formal group law $F$ over $\pi_{0} E$, (which exists because $E$ is complex orientable by properties 2 and 3 , see [1, part 2]), is a universal deformation of its reduction modulo $\mathfrak{m}$.

Thus there is a functor

$$
\mathcal{A}_{\infty} \supset \mathcal{A}_{\infty}^{\mathrm{LT}} \stackrel{\pi}{\longrightarrow} \mathcal{F} \mathcal{G}^{\mathrm{op}}
$$

sending $E \mapsto\left(\pi_{0} E / \mathfrak{m}, F\right)$. 
THEOREM 7.1. The functor $\pi$ is a weak equivalence of topological categories. That is, $\pi$ is surjective on isomorphism classes of $\mathcal{F G}$, and for each $E, F \in \mathcal{A}_{\infty}^{\mathrm{LT}}$ induces a weak equivalence

$$
\operatorname{map}_{\mathcal{A}_{\infty}}(E, F) \rightarrow \mathcal{F} \mathcal{G}(\pi F, \pi E)
$$

where the set on the right hand side is viewed as a space with the discrete topology.

Proof. The theorem will follow from Corollary 14.6 and Corollary 22.7.

Here's how you get Theorem 2.1 from Theorem 7.1. First, choose a small model for the category of formal group laws $\mathcal{F G}$; for example, we could fix an algebraically closed field $K$ of characteristic $p$ and require that $\mathcal{F G}$ only contain those objects $(k, \Gamma)$ for which $k \subset K$. Likewise, choose a small model for $\mathcal{A}_{\infty}^{\mathrm{LT}}$. Now we can perform a "homotopy right Kan extension" of the topological functor $i: \mathcal{A}_{\infty}^{\mathrm{LT}} \rightarrow \mathcal{A}_{\infty}$ along the functor $\pi: \mathcal{A}_{\infty}^{\mathrm{LT}} \rightarrow \mathcal{F} \mathcal{G}^{\mathrm{op}}$, obtaining a functor $\rho: \mathcal{F} \mathcal{G}^{\mathrm{op}} \rightarrow \mathcal{A}_{\infty}$. (Homotopy right Kan extensions are a generalization of the notion of a homotopy inverse limit.) By some general nonsense about homotopy Kan extensions one discovers that $\rho$ has the desired properties; in particular, if $(k, \Gamma)=\pi(E)$, then $\rho(k, \Gamma)$ is weakly equivalent to $E$.

\section{Part 2. Spectra and $\mathcal{A}_{\infty}$-ring spectra}

In the following sections I collect the results we need about spectra and $\mathcal{A}_{\infty}$ ring spectra. I only summarize the facts needed; one should consult the original sources for details and proofs of this material. The category of spectra I use is that of Lewis, May, and Steinberger [9]; in addition to their book, one should also consult the work of Elmendorf, Kriz, Mandell, and May [6], which refines and extends the constructions of [9]. (Note, however, that we make no use the theory of " $S$-modules" which is the main objective of [6].) In addition, I make use results of Hopkins, McClure, and Goerss on the closed model category structure for various categories of spectra; some of this work has not seen print.

I then (in Section 14) set up a spectral sequence and obstruction theory for computing maps between $\mathcal{A}_{\infty}$-ring spectra in the cases we need to consider.

\section{Spectra}

I give a sketch of the theory of spectra of Lewis, May, and Steinberger [9]; see also [6]. I will omit many details.

We work in the category of compactly generated weak Hausdorff spaces, denoted Top. The category of pointed compactly generated weak Hausdorff spaces is denoted $\mathcal{T}$. This means that

$$
\operatorname{map}_{*}(X \wedge Y, Z) \simeq \operatorname{map}_{*}\left(X, \operatorname{map}_{*}(Y, Z)\right),
$$

where $\operatorname{map}_{*}$ denotes the space of pointed maps with an appropriate topology.

A universe $\mathcal{U}$ is a countably dimensional real inner-product space. For example, $\mathbb{R}^{\infty}$ is a universe. An indexing set $\mathcal{A}$ in $\mathcal{U}$ is a collection of finite dimensional vector subspaces of $\mathcal{U}$ such that any finite dimensional subspace of $\mathcal{U}$ is contained in some element of $\mathcal{A}$. Thus, $\mathcal{A}=\left\{\mathbb{R}^{0}, \mathbb{R}^{1}, \mathbb{R}^{2}, \ldots\right\}$ is a indexing set in $\mathbb{R}^{\infty}$. By abuse of notation, we let $\mathcal{U}$ denote the indexing set consisting of all finite dimensional subspaces of $\mathcal{U}$. 
Let $S^{V}$ denote the one-point compactification of the vector space $V$, and let $W-V$ denote the orthogonal complement of $V$ in $W$. A prespectrum on $\mathcal{A}$ is a collection of spaces $X_{V}$ for each $V \in \mathcal{A}$ and maps

$$
i_{V, W}: X_{V} \wedge S^{W-V} \rightarrow X_{W}
$$

for each pair $V, W \in \mathcal{A}$ with $V \subset W$, such that for each $V_{1} \subset V_{2} \subset V_{3}$ the diagram

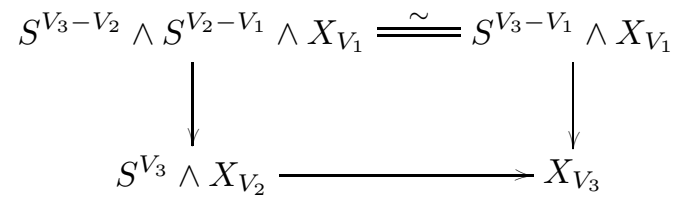

commutes.

A map of prespectra $f: X \rightarrow Y$ is a collection of maps $f_{V}: X_{V} \rightarrow Y_{V}$ commuting with all the structure. We denote the category of prespectra by $\mathcal{P} \mathcal{A}$.

Note that each structure map $i_{V, W}$ is adjoint to a map

$$
\hat{\imath}_{V, W}: X_{V} \rightarrow \Omega^{W-V} X_{W}
$$

where $\Omega^{V} X=\operatorname{map}_{*}\left(S^{V}, X\right)$. A spectrum on $\mathcal{A}$ is a prespectrum $X$ on $\mathcal{A}$ for which each map $\hat{\imath}_{V, W}$ is a homeomorphism. The category of spectra $\mathcal{S} \mathcal{A}$ is the full subcategory of $\mathcal{P} \mathcal{A}$ consisting of the spectra.

Given two prespectra $X$ and $Y$, we can topologize the pointed set of maps $\mathcal{P} \mathcal{A}(X, Y)$ as a subspace of $\prod_{V \in \mathcal{A}} \operatorname{map}_{*}\left(X_{V}, Y_{V}\right)$. It is not hard to see that the composition maps $\mathcal{P} \mathcal{A}(X, Y) \wedge \mathcal{P} \mathcal{A}(Y, Z) \rightarrow \mathcal{P} \mathcal{A}(X, Z)$ are continuous. Thus $\mathcal{P} \mathcal{A}$ is a topological category. Likewise we set $\mathcal{S} \mathcal{A}(X, Y)=\mathcal{P} \mathcal{A}(X, Y)$, making $\mathcal{S} \mathcal{A}$ into a topological category.

First we note that these indexing sets are not too important.

Proposition 8.1. If $\mathcal{A}$ is an indexing set of $\mathcal{U}$ then the functor $\mathcal{S U} \rightarrow \mathcal{S A}$ induced by restriction is an equivalence of categories.

Proof. Given $X \in \mathcal{S A}$, we can produce a spectrum $X^{\prime} \in \mathcal{S U}$ by setting $X_{V}^{\prime}=$ $\Omega^{W-V} X_{W}$ where $W \in \mathcal{A}$ is some subspace of $\mathcal{U}$ containing $V$. It straightforward to check that this is well-defined, and produces the equivalence of categories.

The following proposition says that any prespectrum can be turned into a spectrum. It is crucial and non-trivial.

Proposition 8.2. [9, Appendix] There is a pair of adjoint functors

$$
\ell: \mathcal{P A} \leftrightarrows \mathcal{S A}: r
$$

where $r$ is inclusion of subcategories. Furthermore, $\ell r \simeq 1$, and the functors $\ell$ and $r$ are continuous (i.e., induce continuous maps on function spaces).

As a consequence, it follows that spectra have all small limits and colimits. Prespectra have limits and colimits which are computed space-wise. Limits of spectra can also be computed space-wise, since $\Omega$ commutes with limits of spaces. Colimits of spectra are computed by $\operatorname{colim}_{I} X \simeq \ell X^{\prime}$ where $X^{\prime}$ is the prespectrum defined by $X_{V}^{\prime}=\operatorname{colim}_{I} X_{V}$. 
8.3. Constructions of spectra. Given a space $X$, define a prespectrum $\Sigma_{\mathrm{pre}}^{\infty} X$ by

$$
\left(\Sigma_{\text {pre }}^{\infty} X\right)_{V}=S^{V} \wedge X
$$

with the obvious structure maps, and define the suspension spectrum $\Sigma^{\infty} X$ by $\Sigma^{\infty} X=\ell\left(\Sigma_{\text {pre }}^{\infty} X\right)$. This has the property that

$$
\mathcal{S U}\left(\Sigma^{\infty} X, Y\right) \simeq \operatorname{map}_{*}\left(X, Y_{0}\right)
$$

For each finite $V \subset \mathcal{U}$ we define a prespectrum $S_{\text {pre }}^{-V}$ by

$$
\left(S_{\mathrm{pre}}^{-V}\right)_{W}= \begin{cases}S^{W-V} & \text { if } V \subset W \\ * & \text { otherwise }\end{cases}
$$

with the obvious structure maps. Define a sphere spectrum $S^{-V}$ by $S^{-V}=$ $\ell\left(S_{\text {pre }}^{-V}\right)$. Such an object has the property that

$$
\mathcal{S U}\left(S^{-V}, Y\right) \simeq Y_{V}
$$

For an unpointed space $K$ and a spectrum $X$ over $\mathcal{U}$ we define spectra $K \otimes X$ and $X^{K}$ as follows. Let $X^{K}$ be defined by

$$
X^{K}=\operatorname{map}\left(K, X_{V}\right) \text {. }
$$

It is not hard to check that $X^{K}$ is a spectrum. Let $(K \otimes X)^{\text {pre }}$ be the prespectrum defined by

$$
(K \otimes X)_{V}^{\text {pre }}=K_{+} \wedge X_{V}
$$

and let $K \otimes X=\ell(K \otimes X)^{\text {pre }}$ denote the corresponding spectrum. We have the following fact.

Proposition 8.4. [9, I, Prop. 3.3] Given $K \in$ Top and $X, Y \in \mathcal{S U}$ there are natural isomorphisms

$$
\mathcal{S U}(K \otimes X, Y) \simeq \operatorname{map}(K, \mathcal{S U}(X, Y)) \simeq \mathcal{S U}\left(X, Y^{K}\right)
$$

This means we can define things like homotopies between maps. Given maps $f, g: X \rightarrow Y$ of spectra in $\mathcal{S U}$ a homotopy can be taken to be a map

$$
H: I \rightarrow \mathcal{S U}(X, Y)
$$

such that $H(0)=f$ and $H(0)=g$, or equivalently as a map

$$
H^{\prime}: I \otimes X \rightarrow Y
$$

or a map

$$
H^{\prime \prime}: X \rightarrow Y^{I}
$$

We can think of the object $I \otimes X$ as a cylinder on $X$, and $Y^{I}$ as a spectrum of paths on $Y$. We can use these kinds of constructions to produce mapping-cylinders, path fibrations, and other nifty constructions.

There are constructions analogous to $K \otimes X$ and $X^{K}$ which we can carry out when $K$ is a pointed space. I only give one example: for a pointed space $K$ and spectrum $X$ let $(K \wedge X)^{\text {pre }}$ be the prespectrum defined by

$$
(K \wedge X)_{V}^{\text {pre }}=K \wedge X_{V}
$$

and let $K \wedge X=\ell(K \wedge X)^{\text {pre }}$. Then we have

Proposition 8.5. Given $K \in \mathcal{T}$ and $X, Y \in \mathcal{S U}$ there is a natural isomorphism

$$
\mathcal{S U}(K \wedge X, Y) \simeq \operatorname{map}_{*}(K, \mathcal{S U}(X, Y))
$$


8.6. Model category structure. We say a map $f: X \rightarrow Y$ in $\mathcal{S U}$ is a weak equivalence if for each $V \subset \mathcal{U}$ the induced map $f_{V}: X_{V} \rightarrow Y_{V}$ is a weak equivalence of pointed spaces. We can define homotopy groups of a spectrum $X$ as follows: for each $n \in \mathbb{Z}$, choose a vector space $V \subset \mathcal{U}$ such that $n+\operatorname{dim}(V)=m \geq 0$, and write

$$
\pi_{n}(X)=\pi_{m}\left(X_{V}\right)
$$

One can show that this doesn't depend on the choice of $V$. Thus, a weak equivalence of spectra is just a map which induces isomorphisms on stable homotopy groups.

Proposition 8.7. [6, Thm. 4.4] The category of spectra $\mathcal{S U}$ admits the structure of a Quillen closed model category, such that a map $f: X \rightarrow Y$ is a

1. weak equivalence if each map $f_{V}: X_{V} \rightarrow Y_{V}$ is a weak equivalence of spaces,

2. fibration if each map $f_{V}: X_{V} \rightarrow Y_{V}$ is a Serre fibration,

3. cofibration if $f$ is a retract of a map $f^{\prime}$ which is obtained by successively gluing on "cells" of the form

$$
\partial \Delta_{n} \otimes S^{-V} \rightarrow \Delta_{n} \otimes S^{-V}
$$

where $n \geq 0, V \subset \mathcal{U}$, and $\Delta_{n}$ denotes the Euclidean n-simplex.

Furthermore, this structure gives $\mathcal{S U}$ the structure of a topological model category.

I won't say what all this means. Quillen closed model categories are defined in [11]. The existence of such a structure implies, among other things, that we can form a homotopy category Ho $\mathcal{S U}$ by formally inverting the weak equivalences in $\mathcal{S U}$.

The statement that $\mathcal{S U}$ is a topological model category means that for a cofibration $i: X \rightarrow Y$ and a fibration $p: E \rightarrow F$ of spectra, the induced map of spaces

$$
f: \mathcal{S U}(Y, E) \rightarrow \mathcal{S U}(X, E) \underset{\mathcal{S U}(X, F)}{\times} \mathcal{S U}(Y, F)
$$

is a Serre fibration, and that if either $i$ or $p$ is also a weak equivalence, then so is $f$. (This is analogous to Quillen's notion of a simplicial model category [11].)

\section{Smash products}

In this section I develop the "external" smash product and related constructions.

9.1. Smash product. Given universes $\mathcal{U}$ and $\mathcal{U}^{\prime}$, then the product space $\mathcal{U} \times \mathcal{U}^{\prime}$ is another universe. Let

$$
\mathcal{A}=\left\{V \times W \subset \mathcal{U} \times \mathcal{U}^{\prime} \mid V \subset \mathcal{U}, V^{\prime} \subset \mathcal{U}^{\prime}\right\} .
$$

This is an indexing set of $\mathcal{U} \times \mathcal{U}^{\prime}$. Given $X \in \mathcal{S U}$ and $Y \in \mathcal{U}^{\prime}$ define

$$
(X \wedge Y)_{V \times W}^{\mathrm{pre}}=X_{V} \wedge Y_{W}
$$

this is a prespectrum over $\mathcal{A}$, where the structure maps are

$$
S^{V^{\prime} \times W^{\prime}-V \times W} \wedge X_{V} \wedge Y_{W} \simeq S^{V^{\prime}-V} \wedge S^{W^{\prime}-W} \wedge X_{V} \wedge Y_{W} \rightarrow X_{V^{\prime}} \wedge Y_{W^{\prime}} .
$$

Then let

$$
X \wedge Y=\ell(X \wedge Y)^{\text {pre }} \in \mathcal{S} \mathcal{A} \simeq \mathcal{S}\left(\mathcal{U} \times \mathcal{U}^{\prime}\right) .
$$

Thus we obtain a smash product functor

$$
-\wedge-: \mathcal{S U} \times \mathcal{S U}^{\prime} \rightarrow \mathcal{S}\left(\mathcal{U} \times \mathcal{U}^{\prime}\right) .
$$

As an example, we have 
Proposition 9.2. For $V \subset \mathcal{U}$ and $W \subset \mathcal{U}^{\prime}$ there is a natural isomorphism

$$
S^{-V} \wedge S^{-W} \simeq S^{-V \times W}
$$

of spectra over $\mathcal{U} \times \mathcal{U}^{\prime}$.

Proof. We compute on the level of prespectra, and then spectrify. If $V \subset$ $V^{\prime} \subset \mathcal{U}$ and $W \subset W^{\prime} \subset U^{\prime}$ we get

$$
\left(S^{-V} \wedge S^{-W}\right)_{V^{\prime} \times W^{\prime}}^{\text {pre }} \simeq S^{V^{\prime}-V} \wedge S^{W^{\prime}-W} \simeq S^{V^{\prime} \times W^{\prime}-V \times W} \simeq\left(S^{-V \times W}\right)_{V^{\prime} \times W^{\prime}}^{\text {pre }}
$$

Hence the corresponding spectra are isomorphic.

9.3. Function spectra. Let's fix a $Y \in \mathcal{S} \mathcal{U}^{\prime}$. Then the functor $-\wedge Y: \mathcal{S U} \rightarrow$ $\mathcal{S}\left(\mathcal{U} \times \mathcal{U}^{\prime}\right)$ has a right adjoint, namely the function spectrum

$$
F(Y,-): \mathcal{S}\left(\mathcal{U} \times \mathcal{U}^{\prime}\right) \rightarrow \mathcal{S U}
$$

By this adjoint functor characterization, we see that for $V \subset \mathcal{U}$ we must have

$$
F(Y, Z)_{V} \simeq \mathcal{S U}\left(S^{-V}, F(Y, Z)\right) \simeq \mathcal{S}\left(\mathcal{U} \times \mathcal{U}^{\prime}\right)\left(S^{-V} \wedge Y, Z\right)
$$

and since we have already defined the smash product, we can use this observation to define $F(Y, Z)$ by the above formula. One has that for $V \subset W \subset \mathcal{U}$,

$$
S^{W-V} \wedge S^{-W} \simeq S^{-V}
$$

as one can see by checking this formula on the level of prespectra:

$$
\left(S^{W-V} \wedge S^{-W}\right)_{W^{\prime}}^{\text {pre }} \simeq S^{W-V} \wedge S^{W^{\prime}-W} \simeq S^{W^{\prime}-V} \simeq\left(S_{\text {pre }}^{-V}\right)_{W^{\prime}}
$$

Thus the structure maps of $F(Y, Z)$ are defined to be the natural isomorphisms

$$
\begin{aligned}
F(Y, Z)_{V} & =\mathcal{S}\left(\mathcal{U} \times \mathcal{U}^{\prime}\right)\left(S^{-V} \wedge Y, Z\right) \\
& \simeq \mathcal{S}\left(\mathcal{U} \times \mathcal{U}^{\prime}\right)\left(S^{W-V} \wedge S^{-W} \wedge Y, Z\right) \\
& \simeq \Omega^{W-V} \mathcal{S}\left(\mathcal{U} \times \mathcal{U}^{\prime}\right)\left(S^{-W} \wedge Y, Z\right) \\
& =\Omega^{W-V} F(Y, Z)_{W}
\end{aligned}
$$

and we see that $F(Y, Z)$ is indeed a spectrum.

One can show that if $K$ is an unpointed space and $X$ a spectrum, then $X^{K} \simeq$ $F\left(\Sigma^{\infty} K_{+}, X\right)$.

9.4. Associativity. The significant property of this category of spectra which we need is associativity of the smash product. If $X \in \mathcal{S U}_{1}, Y \in \mathcal{S U}_{2}$ and $Z \in \mathcal{S U}_{3}$, then there is a natural isomorphism

$$
(X \wedge Y) \wedge Z \simeq X \wedge(Y \wedge Z)
$$

of spectra over $\mathcal{U}_{1} \times \mathcal{U}_{2} \times \mathcal{U}_{3}$. In fact, both sides of the above equation are naturally isomorphic to the spectrification of the prespectrum defined by

$$
V_{1} \times V_{2} \times V_{3} \mapsto X_{V_{1}} \wedge Y_{V_{2}} \wedge Z_{V_{3}}
$$

\section{Change of universe functors}

I will talk about several different constructions which allow you to compare spectra which are defined over different universes. Some of the material of this section is in [9, Ch. II] and [6, App. A]. 
10.1. Push-forward and pull-back. Given a linear isometric embedding of universes $f: \mathcal{U} \rightarrow \mathcal{U}^{\prime}$, that is, a vector space map which preserves the inner product, we produce functors

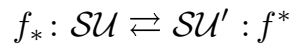

called push-forward and pull-back. Pull-back is easy; for $V \subset \mathcal{U}$ and $Y \in \mathcal{S U}^{\prime}$ let

$$
\left(f^{*} Y\right)_{V}=Y_{f V}
$$

where $f V \subset \mathcal{U}^{\prime}$ denotes the image of $V$ under $f$. The natural homeomorphism $S^{V} \simeq S^{f V}$ induced by $f$ produces isomorphisms

$$
\Omega^{W-V}\left(f^{*} Y\right)_{V} \simeq \operatorname{map}_{*}\left(S^{W-V}, Y_{f V}\right) \simeq \operatorname{map}_{*}\left(S^{f W-f V}, Y_{f V}\right) \simeq Y_{f W}=\left(f^{*} Y\right)_{W} .
$$

Thus $f^{*} Y$ is a spectrum over $\mathcal{U}$.

Push-forward is a little more complicated. We define a pre-spectrum $\left(f_{*} X\right)^{\text {pre }}$ as follows. For $W \subset \mathcal{U}^{\prime}$ let

$$
\left(f_{*} X\right)_{W}^{\mathrm{pre}}=S^{W-f V} \wedge X_{V}
$$

where $V=f^{*}(f V \cap W)$. In other words, $V$ is the largest subspace of $\mathcal{U}$ which maps into $W$. If $W \subset W^{\prime}$ let $V^{\prime}=f^{*}\left(f V^{\prime} \cap W^{\prime}\right)$, whence we get structure maps

$$
\begin{aligned}
S^{W^{\prime}-W} \wedge\left(f_{*} X\right)_{W}^{\text {pre }} \simeq S^{W^{\prime}-W} \wedge S^{W-f V} \wedge X_{V} & \simeq S^{W^{\prime}-f V^{\prime}} \wedge S^{V^{\prime}-V} \wedge X_{V} \\
& \longrightarrow S^{W^{\prime}-V^{\prime}} \wedge X_{V}=\left(f_{*} X\right)_{W^{\prime}}^{\text {pre }}
\end{aligned}
$$

Of course we let $f_{*} X=\ell\left(f_{*} X\right)^{\text {pre }} \in \mathcal{S U}^{\prime}$.

We compute that $f_{*} S^{-V} \simeq S^{-f V}$, by a sequence of adjunctions

$$
\left.\mathcal{S U}^{\prime}\left(f_{*} S^{-V}, Y\right) \simeq \mathcal{S U}\left(S^{-V}, f^{*} Y\right) \simeq\left(f^{*} Y\right)_{V}=Y_{f V} \simeq \mathcal{S U}^{\prime} * S^{-f V}, Y\right) .
$$

Note that if the linear isometry $f: \mathcal{U} \rightarrow \mathcal{U}^{\prime}$ is an isomorphism of universes, then the definition of $f_{*} X$ simplifies to $\left(f_{*} X\right)_{W}=X_{f^{*} W}$, and so $f_{*}$ and $f^{*}$ form an adjoint equivalence of categories.

10.2. Many smash products. We can now use the push-forward functors to define many smash product functors from the category of spectra over $\mathcal{U}$ to itself. Let $\mathcal{U}^{n}=\mathcal{U} \times \cdots \times \mathcal{U}$. Then for $X_{1}, \ldots, X_{n} \in \mathcal{S U}$ and for some linear isometric map $f: \mathcal{U}^{n} \rightarrow \mathcal{U}$ we define a functor $\mathcal{S} \mathcal{U}^{n} \rightarrow \mathcal{S U}$ by

$$
X_{1}, \ldots, X_{n} \mapsto f_{*}\left(X_{1} \wedge \cdots \wedge X_{n}\right) .
$$

Note that if $i: \mathcal{U}_{1} \rightarrow \mathcal{U}_{2}$ and $j: \mathcal{U}_{2} \rightarrow \mathcal{U}_{3}$ then $(j \circ i)_{*} X \simeq j_{*} i_{*} X$. Thus, given $f: \mathcal{U}^{2} \rightarrow \mathcal{U}$ and $g: \mathcal{U}^{2} \rightarrow \mathcal{U}$ we can compose smash product functors, so that

$$
f_{*}\left(g_{*}(X \wedge Y) \wedge Z\right) \simeq(f \circ(g \times 1))_{*}(X \wedge Y \wedge Z)
$$

where $f \circ(g \times 1)): \mathcal{U}^{3} \rightarrow \mathcal{U}$ denotes the composite map on universes.

10.3. "Twisted" constructions involving the linear isometries space. Let $\mathcal{L}\left(\mathcal{U}, \mathcal{U}^{\prime}\right)$ denote the set of all linear isometries from $\mathcal{U}$ to $\mathcal{U}^{\prime}$. Topologize this space by the compact-open topology. Then one has the following result.

Proposition 10.4. [9, II, Lemma 1.5] The space $\mathcal{L}\left(\mathcal{U}, \mathcal{U}^{\prime}\right)$ is contractible. 
For us, this means that we have a space $\mathcal{L}\left(\mathcal{U}^{n}, \mathcal{U}\right)$ which parameterizes all possible ways of smashing $n$ things together, and this parameter space is contractible. One would like to somehow glue "all" these smash-products together and get a super-universal smash product functor from $\mathcal{S U}$ to itself.

For the time being let's write $\mathcal{L}$ for $\mathcal{L}\left(\mathcal{U}, \mathcal{U}^{\prime}\right)$. For maps of topological spaces $T \rightarrow \mathcal{L}$ and $T^{\prime} \rightarrow \mathcal{L}$ let $\operatorname{map}_{\mathcal{L}}\left(T, T^{\prime}\right) \subset \operatorname{map}\left(T, T^{\prime}\right)$ denote the space of maps $T \rightarrow T^{\prime}$ which commute with the projections to $\mathcal{L}$. Let Top denote the category of unpointed spaces, and let (Top $\downarrow \mathcal{L})$ denote the category of spaces over $\mathcal{L}$.

Proposition 10.5. Given $t: T \rightarrow \mathcal{L} \in($ Top $\downarrow \mathcal{L}), X \in \mathcal{S U}$ and $Y \in \mathcal{S U}^{\prime}$ there exist functorial constructions

- $T \rtimes X \in \mathcal{S U}^{\prime}$,

- $Y^{T} \in \mathcal{S U}$,

- $\mathcal{S}(X, Y) \rightarrow \mathcal{L} \in(\operatorname{Top} \downarrow \mathcal{L})$,

such that there are natural isomorphisms

$$
\mathcal{S U}^{\prime}(T \rtimes X, Y) \simeq \operatorname{map}_{\mathcal{L}}(T, \mathcal{S}(X, Y)) \simeq \mathcal{S U}\left(X, Y^{T}\right),
$$

and such that if $T=\{f\} \rightarrow \mathcal{L}$ is a single point, then

- $\{f\} \rtimes X \simeq f_{*} X$,

- $Y^{\{f\}} \simeq f^{*} Y$, and

- $\operatorname{map}_{\mathcal{L}}(\{f\}, \mathcal{S}(X, Y)) \simeq \mathcal{S U}^{\prime}\left(f_{*} X, Y\right) \simeq \mathcal{S U}\left(X, f^{*} Y\right)$, or in other words, the fiber of the map $\mathcal{S}(X, Y) \rightarrow \mathcal{L}(\mathcal{U}, \mathcal{V})$ over the point $f$ is homeomorphic to the above spaces.

The construction $T \rtimes X$ is known as the twisted half-smash product.

First I'll outline a construction of $T \rtimes X$. First, suppose $T$ is a compact space. For $V \subset \mathcal{U}$ let $T(V) \subset \mathcal{U}^{\prime}$ denote the union of all $f V \subset \mathcal{U}^{\prime}$ as $f$ ranges over all elements in the image of $\xi: T \rightarrow \mathcal{L}$. Since $T$ is compact there exists a finite dimensional vector subspace $W \subset \mathcal{U}^{\prime}$ such that $T(V) \subset W$. Conversely, for each $W \subset \mathcal{U}^{\prime}$ there is a largest $V \subset \mathcal{U}$ such that $T(V) \subset W$.

Given such $V$ and $W$ with $T(V) \subset W$, let $\xi(V) \rightarrow T$ denote the vector subbundle of the trivial bundle $W \times T \rightarrow T$ such that over $t \in T$ the fiber is $\xi(t)(V) \subset$ $W$. Let $W-\xi(V)$ denote the orthogonal complement bundle of $\xi(V)$ in $W \times T$, and let $T^{W-\xi(V)}$ denote the Thom space. Define

$$
(T \rtimes X)_{W}^{\mathrm{pre}}=T^{W-\xi(V)} \wedge X_{V} .
$$

Suppose $W \subset W^{\prime} \subset \mathcal{U}^{\prime}$. Then there are corresponding spaces $V \subset V^{\prime} \subset \mathcal{U}$ and sub-bundles $\xi(V) \subset \xi\left(V^{\prime}\right)$ of $W^{\prime} \times T$. Then

$$
\left(W^{\prime}-W\right) \times(W-\xi(V)) \simeq W^{\prime}-\xi(V) \simeq\left(W^{\prime}-\xi\left(V^{\prime}\right)\right) \times\left(V^{\prime}-V\right)
$$

since $\xi\left(V^{\prime}\right)-\xi(V)$ can be identified with a trivial bundle with fiber $V^{\prime}-V$. Thus structure maps are defined by

$$
\begin{aligned}
S^{W^{\prime}-W} \wedge(T \rtimes X)_{W}^{\mathrm{pre}} & \simeq S^{W^{\prime}-W} \wedge T^{W-\xi(V)} \wedge X_{V} \\
& \simeq T^{W^{\prime}-\xi\left(V^{\prime}\right)} \wedge S^{V^{\prime}-V} \wedge X_{V} \\
& \rightarrow T^{W^{\prime}-\xi\left(V^{\prime}\right)} \wedge X_{V^{\prime}}=(T \rtimes X)_{W^{\prime}}^{\text {pre }}
\end{aligned}
$$

Of course, we now let $T \rtimes X=\ell(T \rtimes X)^{\text {pre }}$. Note that if $T=\{f\} \in \mathcal{L}$ then we have just recovered the construction of $f_{*} X$. 
If $T$ is not compact, then we let

$$
T \rtimes X=\operatorname{colim}_{K \subset T} K \rtimes X
$$

as $K$ ranges over all the compact subspaces of $T$.

For $\mathcal{S}(X, Y)$, we let this be equal as a set to

$$
\mathcal{S}(X, Y)=\coprod_{f \in \mathcal{L}} \mathcal{S U}\left(X, f^{*} Y\right)
$$

This has a natural map $\pi: \mathcal{S}(X, Y) \rightarrow \mathcal{L}$. We topologize it by giving it the largest topology such that a map $f: T \rightarrow \mathcal{S}(X, Y)$ is continuous map if and only if

1. the composite $\pi f: T \rightarrow \mathcal{L}$ is continuous, and

2. the corresponding map $T \rtimes X \rightarrow Y$ is a map of spectra.

This last is a construction of Elmendorf [5].

10.6. Applications of the twisted constructions. We need one more set of facts about all these constructions; namely, that they preserve features of the model category structure as best as is possible. Since I haven't been explicit about model category structures I won't give the general statement, but here are some consequences.

1. If $T \rightarrow \mathcal{L}$ with $T$ a $\mathrm{CW}$-complex, and $X \in \mathcal{S U}$ a cofibrant spectrum, then $T \rtimes X \in \mathcal{S U}^{\prime}$ is cofibrant.

2. If $T \stackrel{f}{\rightarrow} T^{\prime} \rightarrow \mathcal{L}$ is such that $f$ is a weak equivalence between $\mathrm{CW}$-complexes, and $X \in \mathcal{S U}$ is a cofibrant spectrum, then $T \rtimes X \rightarrow T^{\prime} \rtimes X$ is a weak equivalence of spectra over $\mathcal{U}^{\prime}$.

3. If $T \rightarrow \mathcal{L}$ with $T$ a $\mathrm{CW}$-complex, and $X \rightarrow Y$ a weak equivalence between cofibrant spectra over $\mathcal{U}$, then $T \rtimes X \rightarrow T \rtimes Y$ is a weak equivalence of spectra over $\mathcal{U}^{\prime}$.

4. If $X \in \mathcal{S U}$ is a cofibrant spectrum, and $Y \rightarrow Y^{\prime} \in \mathcal{S \mathcal { U } ^ { \prime }}$ is a weak equivalence of cofibrant spectra, then $X \wedge Y \rightarrow X \wedge Y^{\prime}$ is a weak equivalence of spectra over $\mathcal{U} \times \mathcal{U}^{\prime}$.

5. If $X \in \mathcal{S U}$ is a cofibrant spectrum, and $Y \in \mathcal{S U}^{\prime}$, then $\mathcal{S}(X, Y) \rightarrow \mathcal{L}$ is a Serre fibration.

Corollary 10.7. If $f, g: \mathcal{U} \rightarrow \mathcal{U}^{\prime}$ are linear isometries, and $X \in \mathcal{S U}$ a cofibrant spectrum, then $f_{*} X$ and $g_{*} Y$ are weakly equivalent as spectra over $\mathcal{U}^{\prime}$, and furthermore this weak equivalence may be chosen canonically up to homotopy.

Proof. Chose a path $\gamma: I \rightarrow \mathcal{L}$ between $f$ and $g$ (recall that $\mathcal{L}$ is contractible). Then we get a diagram

$$
f_{*} X \simeq\{f\} \rtimes X \rightarrow I \rtimes X \leftarrow\{g\} \rtimes X
$$

where each of the maps is a weak equivalence by (2) above, since the unit interval $I$ is certainly a CW-complex. Similarly, given two homotopies $\gamma$ and $\gamma^{\prime}$, an argument involving homotopies between homotopies shows that the weak equivalence is canonical up to homotopy.

COROLlary 10.8. For any two universes $\mathcal{U}$ and $\mathcal{U}^{\prime}$, the homotopy categories of spectra $\mathrm{Ho} \mathcal{S U}$ and $\mathrm{Ho}_{\mathcal{S U}}$ are canonically equivalent.

Corollary 10.9. For any cofibrant $X, Y \in \mathcal{S U}$ and $f, g \in \mathcal{L}\left(\mathcal{U}^{2}, \mathcal{U}\right)$ the smash products $f_{*}(X \wedge Y)$ and $g_{*}(X \wedge Y)$ are canonically weakly equivalent, and thus there is a canonical smash product defined on the level of the homotopy category. 


\section{Operads}

I give a couple of definitions of operads.

11.1. Definition of operads. An operad in Top is a collection of spaces $\{C[n]\}_{n \geq 0}$ equipped with the following structure:

1. a right action of $\Sigma_{n}$ on $C[n]$,

2. a distinguished point $1 \in C[1]$,

3. and for each sequence $I=\left(i_{1}, \ldots, i_{k}\right)$ of non-negative integers $(k \geq 0)$, and $i_{1}+\cdots+i_{k}=n$ a map

$$
C[k] \times C\left[i_{1}\right] \times \cdots \times C\left[i_{k}\right] \rightarrow C[n] .
$$

Notationally, this map sends $f \in C[k], g_{j} \in C\left[i_{j}\right]$ to $f\left\{g_{1}, \ldots, g_{k}\right\}$.

In addition, the following relations must hold.

1. $1\{g\}=g$ for $g \in C[n]$,

2. $f\{1, \ldots, 1\}=f$ for $f \in C[k]$,

3. for $f \in C[k], g_{j} \in C\left[i_{j}\right]$, and $h_{j, \ell} \in C\left[m_{j, \ell}\right]$, $f\left\{g_{1}\left\{h_{1,1}, \ldots, h_{1, m_{1}}\right\}, \ldots, g_{k}\left\{h_{k, 1}, \ldots, h_{k, m_{k}}\right\}=\left(f\left\{g_{1}, \ldots, g_{k}\right\}\right)\left\{h_{1,1}, \ldots, h_{k, m_{k}}\right\}\right\}$,

4. $(f \sigma)\left\{g_{1}, \ldots, g_{k}\right\}=f\left\{g_{\sigma 1}, \ldots, g_{\sigma k}\right\}$ for $f \in C[k], g_{j} \in C\left[i_{j}\right]$, and $\sigma \in \Sigma_{k}$,

5. $f\left\{g_{1} \sigma_{1}, \ldots, g_{k} \sigma_{k}\right\}=f\left\{g_{1}, \ldots, g_{k}\right\} \sigma_{1} \times \cdots \times \sigma_{k}$ for $f \in C[k], g_{j} \in C\left[i_{j}\right]$, and $\sigma_{j} \in \Sigma_{i_{j}}$.

A map of operads $C \rightarrow D$ is a collection of continuous maps $C[n] \rightarrow D[n]$ which commute with all the structure.

11.2. Examples of operads. Given a space $X \in$ Top, define $\mathcal{E}_{X}$ by

$$
\mathcal{E}_{X}[n]=\operatorname{map}\left(X^{n}, X\right) .
$$

It is not hard to see that $\mathcal{E}_{X}$ is an operad, under composition of functions. It is called the endomorphism operad of $X$. Likewise, the spaces $\mathcal{L}[n]=\mathcal{L}\left(\mathcal{U}^{n}, \mathcal{U}\right)$ also form an operad under composition, called the linear isometries operad.

Another example of an operad is the associative operad. Let $A[n]$ denote the set of monomials of length $n$ in $n$ distinct non-commuting formal variables $t_{1}, \ldots, t_{n}$. E.g., $A[2]=\left\{t_{1} t_{2}, t_{2} t_{1}\right\}$. Write $f\left(t_{1}, \ldots, t_{n}\right)$ for a general element of $A[n]$, and write $1 \in A[0]$ for the "empty" monomial, (not to be confused with the distinguished element of $A[1]$, which is denoted $t_{1}$ ). Then $A$ is an operad, under "composition" of formal monomials; that is,

$$
\begin{aligned}
& f\left\{g_{1}, \ldots, g_{k}\right\}\left(t_{1}, \ldots, t_{n}\right) \\
& \quad=f\left(g_{1}\left(t_{1}, \ldots, t_{i_{1}}\right), g_{2}\left(t_{i_{1}+1}, \ldots, t_{i_{1}+i_{2}}\right), \ldots, g_{k}\left(t_{i_{1}+\cdots+i_{k-1}+1}, \ldots, t_{n}\right)\right) .
\end{aligned}
$$

If $X$ is a topological monoid, then you can produce a map $A \rightarrow \mathcal{E}_{X}$ of operads by evaluation, i.e.,

$$
f\left(t_{1}, \ldots, t_{n}\right) \mapsto\left(\left(x_{1}, \ldots, x_{n}\right) \mapsto f\left(x_{1}, \ldots, x_{n}\right)\right) .
$$

In fact, giving the structure of a topological monoid on $X$ is precisely equivalent to giving a map of operads from $A$ to $\mathcal{E}_{X}$. 
11.3. $\Sigma$-objects. I need a description of operads which shows off some of their other facets, and will be useful in Section 23. This definition works in any symmetric monoidal category. The primary example of interest here is the category of topological spaces, with cartesian product as monoidal product. To keep things simple, I state the definitions in terms of the category Top. But one should keep in mind that things work in a general symmetric monoidal category.

A $\Sigma$-object $C$ is a collection of spaces $\{C[n]\}_{n \geq 0}$ such that $C[n]$ is equipped with a right action by $\Sigma_{n}$. Thus, any operad is a $\Sigma$-object, but not conversely.

Given a $\Sigma$-object $C$, define a functor $F_{C}$ : Top $\rightarrow$ Top by

$$
F_{C}(X)=\coprod_{n \geq 0} C[n] \underset{\Sigma_{n}}{\times} X^{n} .
$$

Thus each $\Sigma$-object produces a functor; likewise, each map of $\Sigma$-objects produces a natural transformation of functors. It is sometimes helpful to think of $F_{C}$ as a "power series" with coefficients $C[n]$.

Let $I$ be the $\Sigma$-object defined by

$$
I[n]= \begin{cases}\mathrm{pt} & \text { if } n=1, \\ \varnothing & \text { otherwise }\end{cases}
$$

Then $F_{I}$ is the identity functor.

Suppose $D$ is another $\Sigma$-object. What is $F_{C}(X) \times F_{D}(X)$ ? We compute

$$
\begin{aligned}
F_{C}(X) \times F_{D}(X) & \simeq\left(\coprod_{m \geq 0} C[m] \underset{\Sigma_{m}}{\times} X^{m}\right) \times\left(\coprod_{n \geq 0} D[n] \underset{\Sigma_{n}}{\times} X^{n}\right) \\
& \simeq \coprod_{m, n \geq 0}(C[m] \times D[n]) \underset{\Sigma_{m} \times \Sigma_{n}}{\times}\left(X^{m} \times X^{n}\right) \\
& \simeq \coprod_{d \geq 0}\left(\coprod_{m+n=d} C[m] \times D[n] \underset{\Sigma_{m} \times \Sigma_{n}}{\left.\underset{\Sigma_{d}}{\times}\right) \underset{\Sigma_{d}}{\times} X^{d} .}\right.
\end{aligned}
$$

Thus, if we define a new $\Sigma$-object $C \otimes D$ by

$$
(C \otimes D)[d]=\coprod_{m+n=d} C[m] \times D[n] \underset{\Sigma_{m} \times \Sigma_{n}}{\times} \Sigma_{d}
$$

then we see that $F_{C}(X) \times F_{D}(X) \simeq F_{C \otimes D}(X)$. Note that this implies that $F_{C}(X)^{\times n} \simeq F_{C \otimes n}(X)$.

We can produce other formulas of the same type. Thus, if $K$ is a space, define a $\Sigma$-object $K \times C$ by $(K \times C)[n]=K \times C[n]$. Then $K \times F_{C}(X) \simeq F_{K \times C}(X)$. Also, if $C_{\alpha}$ is a diagram of $\Sigma$-objects, then $\operatorname{colim}\left(F_{C_{\alpha}}(X)\right) \simeq F_{\text {colim } C_{\alpha}}(X)$.

The formula we are really after is the one for the composition of functors $F_{C}\left(F_{D}(X)\right)$. We compute

$$
\begin{aligned}
& F_{C}\left(F_{D}(X)\right) \simeq \coprod_{m \geq 0} C[m] \underset{\Sigma_{m}}{\times} F_{D}(X)^{m} \\
& \simeq \coprod_{m \geq 0} C[m] \underset{\Sigma_{m}}{\times} F_{D^{\otimes m}}(X)
\end{aligned}
$$

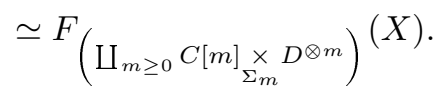


Thus, if we set

$$
C \circ D=\coprod_{m \geq 0} C[m] \underset{\Sigma_{m}}{\times} D^{\otimes m}
$$

then we have $F_{C} \circ F_{D} \simeq F_{C \circ D}$. In full, this means

$$
(C \circ D)[d]=\coprod_{m \geq 0} C[m] \underset{\Sigma_{m}}{\times}\left(\coprod_{i_{1}+\cdots+i_{m}=d} D\left[i_{1}\right] \times \cdots \times D\left[i_{m}\right] \underset{\Sigma_{i_{1}} \times \cdots \times \Sigma_{i_{m}}}{\times} \underset{\Sigma_{d}}{\times}\right) .
$$

This makes the category of $\Sigma$-objects into a monoidal category, where the monoidal structure corresponds to composition of functors.

11.4. Operads are monoids. Since the category of $\Sigma$-object is a monoidal category via the "०" product (but not symmetric monoidal, since $C \circ D \neq D \circ C$ ), we can think about monoids in this category. A monoid is just a $\Sigma$-object $C$ equipped with maps $\eta: I \rightarrow C$ and $\mu: C \circ C \rightarrow C$ such that the following diagrams commute.
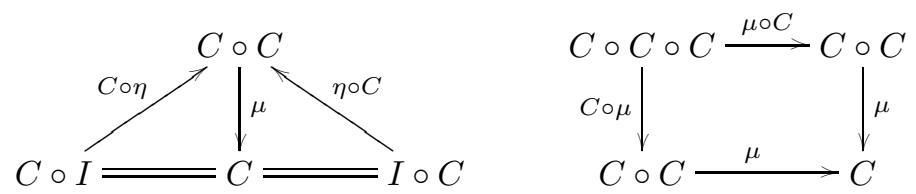

Proposition 11.5. The category of operads is equivalent to the category of ¿-object-monoids.

Proof. Suppose $C$ is a $\Sigma$-object-monoid. Then the map $\eta: I \rightarrow C$ is just the choice of a distinguished point in $C[1]$, and the multiplication map $\mu: C \circ C \rightarrow C$ amounts to choosing a collection of maps

$$
C[m] \times C\left[i_{1}\right] \times \cdots \times C\left[i_{m}\right] \rightarrow C[d]
$$

which satisfy axioms (4) and (5) for an operad. The three remaining axioms (1), (2), and (3) follow from the fact that $C$ is a monoid. The converse argument is similar.

A $\Sigma$-object monoid $C$ defines a functor $F_{C}:$ Top $\rightarrow$ Top which is a triple. Thus, given an operad $C$, we can speak of algebras over the triple $F_{C}$.

Proposition 11.6. The category of $C$-algebras is equivalent to the category of $F_{C}$-algebras.

This implies in particular that for a space $X, F_{C}(X)=\coprod C[m] \underset{\Sigma_{m}}{\times} X^{n}$ is a $C$-algebra. In fact, it is the free $C$-algebra on $X$, with the property that

$$
C-\operatorname{alg}\left(F_{C}(X), Y\right) \simeq \operatorname{map}(X, Y) .
$$

For example, if $A$ is the associative operad,

$$
F_{A}(X) \simeq \coprod_{n \geq 0} X^{n}
$$

\section{Algebra spectra}

We discuss spectra which are also algebras over an operad. 
12.1. Operads over the linear isometries operad. We want to have operads acting on spectra, rather than on spaces. First, note that there's a natural inclusion $\Sigma_{n} \subset \mathcal{L}\left(\mathcal{U}^{n}, \mathcal{U}^{n}\right)$. Let $X$ be a spectrum over $\mathcal{U}$. For each $\sigma \in \Sigma_{n}$ there's a natural isomorphism $X^{(n)} \simeq \sigma_{*} X^{(n)}$ as follows. Recall that

$$
\left(X_{\text {pre }}^{(n)}\right)_{V_{1} \times \cdots V_{n}}=X_{V_{1}} \wedge \cdots \wedge X_{V_{n}} \text {. }
$$

This means that

$$
\left(\sigma_{*} X^{(n)}\right)_{V_{1} \times \cdots V_{n}}^{\mathrm{pre}} \simeq X_{V_{\sigma 1}} \wedge \cdots \wedge X_{V_{\sigma n}} .
$$

There's a natural map $\left(X^{(n)}\right)_{\text {pre }} \rightarrow\left(\sigma_{*} X^{(n)}\right)_{\text {pre }}$ induced by permuting the factors, which is an isomorphism. This specifies the desired isomorphism of spectra.

The space $\mathcal{L}[n]$ gets a right $\Sigma_{n}$-action by permuting the source universe $\mathcal{U}^{n}$. Suppose $T \rightarrow \mathcal{L}[n]$ is a map of $\Sigma_{n}$-spaces. Then $T \rtimes X^{(n)}$ has a natural $\Sigma_{n}$-action, where $\sigma \in \Sigma_{n}$ acts via the composite of isomorphisms

$$
T \rtimes X^{(n)} \simeq T \rtimes \sigma_{*} X^{(n)} \simeq T \sigma \rtimes X^{(n)} \simeq T \rtimes X^{(n)},
$$

where $T \sigma$ denotes the space over $\mathcal{L}[n]$ defined by the composite map $T \rightarrow \mathcal{L}[n] \stackrel{\sigma}{\rightarrow}$ $\mathcal{L}[n]$, and the right-hand map in the sequence is induced by the isomorphism $T \sigma \rightarrow$ $T$ of spaces over $\mathcal{L}[n]$.

By a $\Sigma$-object over the linear isometries operad, I mean a map $C \rightarrow \mathcal{L}$ of $\Sigma$-objects. For $X \in \mathcal{S U}$ let $F_{C}(X)$ denote the spectrum

$$
F_{C}(X)=\bigvee_{n \geq 0} C[n] \underset{\Sigma_{n}}{\rtimes} X^{(n)}
$$

Thus $F_{C}: \mathcal{S U} \rightarrow \mathcal{S U}$ is a functor. (Note: $C[0] \rtimes X^{(0)}$ is defined to be $\Sigma^{\infty} C[0]_{+}$.)

Given an operad over the linear isometries operad, i.e., an operad map $C \rightarrow \mathcal{L}$, the functor $F_{C}$ is a triple on $\mathcal{S U}$, and we can speak of the category of algebras over $C$.

One example of an operad over $\mathcal{L}$ is the endomorphism operad of $X$. Define the endomorphism "operad" of a spectrum $X$ to be an operad over $\mathcal{L}$, by

$$
\mathcal{E}_{X}[n]=\mathcal{S}\left(X^{(n)}, X\right) \rightarrow \mathcal{L}[n] .
$$

Then one can show that a $C$-algebra structure on $X$ is precisely the same as a map of operads $C \rightarrow \mathcal{E}_{X}$ making the diagram

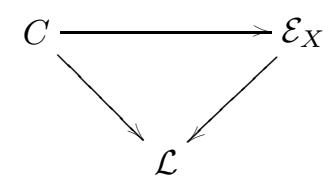

commute.

The main examples of operads we'll need are $\mathcal{A}_{\infty}$-operads. An $\mathcal{A}_{\infty}$ operad $A$ is one for which the operad $\pi_{0} A$ of path components, defined by $\left(\pi_{0} A\right)[n]=\pi_{0} A[n]$, is isomorphic to the associative operad, and each component of $A[n]$ is contractible. We call a spectrum which is an algebra over such an operad an $\mathcal{A}_{\infty}$-ring spectrum.

For example, there is an operad with $A[n]=\mathcal{L}[n] \times \Sigma_{n}$ which is an operad over the linear isometries operad; it induces a functor

$$
F_{A}(X) \simeq \bigvee_{n \geq 0} \mathcal{L}[n] \rtimes X^{(n)} .
$$


In $[6]$ the authors use this operad $A$ to define their notion of $\mathcal{A}_{\infty}$-ring spectrum. It will be necessary for us to use a different $\mathcal{A}_{\infty}$-operad which has technical properties we will need. As will be noted below, this is not a major problem.

Note that if $X$ is a spectrum, then $F_{C}(X)$ naturally is a $C$-algebra, and is in fact the free $C$-algebra on $X$, I'll denote the free $C$-algebra by $C(X)$. If $Y$ is a $C$-algebra, I write $U Y$ for its underlying spectrum.

12.2. Properties of $C$-algebra spectra. The category of $C$-algebras has properties very much like those of the category of spectra $\mathcal{S U}$. I'll summarize them.

1. $C$-alg is a topological category. That is, for every two $C$-algebras $X$ and $Y$ the set of $C$-algebra maps $C$ - $\operatorname{alg}(X, Y) \subset \mathcal{S U}(X, Y)$ can be topologized as a subspace of the space of spectrum maps.

2. The category of $C$-algebras has all small limits and colimits. Colimits of $C$-algebras can be quite complicated. But note that limits are easy. For example, if $X$ and $Y$ are $C$-algebras, and $U X$ and $U Y$ denote the underlying spectra of $X$ and $Y$, then $U X \times U Y$ admits a natural $C$-algebra structure given by

$$
F_{C}(U X \times U Y) \rightarrow F_{C} U X \times F_{C} U Y \rightarrow U X \times U Y .
$$

This is the product in the category of $C$-algebras. Thus, limits of $C$-algebras are computed on the underlying spectrum.

3. Given an unpointed space $T$ and $C$-algebras $X$ and $Y$, there are constructions $T \underset{C}{\otimes} X$ and $Y^{T}$, with the property that

$$
C-\operatorname{alg}(T \underset{C}{\otimes} X, Y) \simeq \operatorname{map}(T, C-\operatorname{alg}(X, Y)) \simeq C-\operatorname{alg}\left(X, Y^{T}\right) .
$$

Furthermore, $U\left(Y^{T}\right) \simeq(U Y)^{T}$. (But note that $\left.U(T \otimes X) \not T \otimes U X !\right)$ Let $E$ be a $C$-algebra. Then $\pi_{*}(U E)^{T}=\pi_{*} U\left(E^{T}\right) \simeq E^{-*} T$. Thus, if $E$ is an $\mathcal{A}_{\infty}$-ring spectrum, then $E^{T}$ is an $\mathcal{A}_{\infty}$-ring spectrum whose homotopy is the cohomology of $T$.

4. The category of $C$-algebra spectra is a topological closed model category (this is non-trivial to prove). The weak equivalences and fibrations are taken to be those maps which are weak equivalences or fibrations on the underlying spectra.

Some of these results appear in EKMM [6]. The final result on the model category structure of $C$-algebras represents work of Hopkins. (EKMM prove the model category structure for certain special operads $C$, but not the general case.)

We note one more result, also due to Hopkins: the homotopy category of $C$ algebra spectra depends (up to equivalence) only on the weak homotopy type of the operad $C$. In particular, this means that all $\mathcal{A}_{\infty}$-operads give the same homotopy category of algebra spectra. Thus, we are always free to replace a given $\mathcal{A}_{\infty}$ operad $A$ with an $A^{\prime}$ which is technically convenient for us.

\section{Simplicial spectra}

Let $\boldsymbol{\Delta}$ denote the category having as objects the finite ordered sets $[n]=$ $\{0,1, \ldots, n\}$, and as morphisms those set maps $f:[m] \rightarrow[n]$ for which $i \leq j$ implies $f(i) \leq f(j)$. This category has a representation in Top which is easier to picture: there's a functor $\Delta: \Delta \rightarrow$ Top which sends $[n]$ to the Euclidean $n$-simplex 
$\Delta_{n}$ which has vertices labelled $v_{0}, v_{1}, \ldots, v_{n}$, and which sends $f:[m] \rightarrow[n]$ to the affine map which sends $v_{i}$ to $v_{f(i)}$.

A simplicial object in a category $\mathbf{C}$ is a functor $X_{\bullet}: \boldsymbol{\Delta}^{\mathrm{op}} \rightarrow \mathbf{C}$, and we call the category of such $s \mathbf{C}$. We define a realization functor $|-|: s \mathcal{S U} \rightarrow \mathcal{S U}$ as a quotient

$$
\coprod_{n \geq 0} \Delta_{n} \otimes X_{n} / \sim
$$

More precisely, it is the coequalizer of

$$
\coprod_{[m] \rightarrow[n] \in \Delta} \Delta_{m} \otimes X_{n} \rightrightarrows \coprod_{[d] \in \Delta} \Delta_{d} \otimes X_{d}
$$

in which the summand corresponding to $\delta:[m] \rightarrow[n] \in \boldsymbol{\Delta}$ is mapped by the top arrow to $\delta \otimes X_{n}$, and by the bottom arrow to $\Delta_{m} \otimes \delta$. Geometric realization is characterized by the fact that it is the left adjoint to a functor $\mathcal{S U} \rightarrow s \mathcal{S U}$ sending $Y$ to $[n] \mapsto Y^{\Delta_{n}}$.

An augmented simplicial object is a simplicial object $X$ • together with a map $\epsilon: X_{0} \rightarrow Y$ such that the two maps $X_{1} \rightrightarrows X_{0} \rightarrow Y$ are the same. Thus, if $X_{\bullet} \rightarrow Y$ is an augmented simplicial spectrum, then there's an induced map $\left|X_{\bullet}\right| \rightarrow Y$.

13.1. Facts about geometric realizations of simplicial spectra. We need the following facts $[\mathbf{6}]$.

1. If $X_{\bullet} \in s \mathcal{S U}$ and $Y_{\bullet} \in s \mathcal{S U}^{\prime}$, then you can form a simplicial spectrum $X_{\bullet} \wedge Y_{\bullet}$ in $s \mathcal{S}\left(\mathcal{U} \times \mathcal{U}^{\prime}\right)$, defined by $[n] \mapsto X_{n} \wedge Y_{n}$. The crucial fact is that there's a natural isomorphism of spectra

$$
\left|X_{\bullet} \wedge Y_{\bullet}\right| \simeq\left|X_{\bullet}\right| \wedge\left|Y_{\bullet}\right|
$$

2. If $T \rightarrow \mathcal{L}\left(\mathcal{U}, \mathcal{U}^{\prime}\right)$ and $X_{\bullet} \in s \mathcal{S U}$, then there's a natural isomorphism

$$
\left|T \rtimes X_{\bullet}\right| \simeq T \rtimes\left|X_{\bullet}\right| \text {. }
$$

3. Geometric realization commutes with colimits.

So if $C \rightarrow \mathcal{L}$ is a $\Sigma$-object over $\mathcal{L}$, then there's a natural isomorphism

$$
F_{C}\left|X_{\bullet}\right| \simeq\left|F_{C}\left(X_{\bullet}\right)\right| \text {. }
$$

Now suppose $X_{\bullet}$ is a simplicial $C$-algebra for some operad $C$, i.e., a functor $X_{\bullet}: \boldsymbol{\Delta}^{\mathrm{op}} \rightarrow C$-alg. Let $U X$. denote the underlying simplicial spectrum. Then we can make $\left|U X_{\bullet}\right|$ into a $C$-algebra, by a map

$$
F_{C}\left|U X_{\bullet}\right| \simeq\left|F_{C} U X_{\bullet}\right| \rightarrow\left|U X_{\bullet}\right| .
$$

So the geometric realization of a simplicial $C$-algebra is again a simplicial $C$-algebra.

13.2. Resolutions of a $C$-algebra. Given a $C$-algebra $X$, let $C X$ denote the free $C$-algebra on the underlying spectrum of $X$, so $U C X \simeq \bigvee_{n \geq 0} C[n] \underset{\Sigma_{n}}{\rtimes} X^{(n)}$. Then we get an augmented simplicial object

$$
X \leftarrow C X \leftleftarrows C C X \cdots
$$

which I'll denote by $C^{\bullet+1} X$. (By abuse of notation I omit the forget functor $U$.)

We need a model category fact: for good (i.e., cofibrant) choices of $X$ and $C$, the natural map $\left|C^{\bullet+1} X\right| \rightarrow X$ is a weak equivalence of $C$-algebras, and $\left|C^{\bullet+1} X\right|$ is 
itself a cofibrant $C$-algebra, and that $C^{\bullet+1} X$ is "Reedy cofibrant" in the category of simplicial $C$-algebras. This means in particular that

$$
C-\operatorname{alg}(X, E) \rightarrow C-\operatorname{alg}\left(\left|C^{\bullet+1} X\right|, E\right)
$$

is a weak equivalence of spaces. We will compute $\mathcal{A}_{\infty}$ maps between $\mathcal{A}_{\infty}$-ring spectra using a spectral sequence based on this resolution, using an $\mathcal{A}_{\infty}$-operad $C$.

REMARK 13.3. It is important that we be able to produce a Reedy cofibrant resolution, as we will see below. In order to produce such it is convenient to have an operad $C$ for which a cofibrant $C$-algebra $X$ has an underlying spectrum $U X$ which is cofibrant as a spectrum. It turns out that this will be the case when $C$ is a cofibrant operad; these will be discussed in Section 22.2. Thus, we will assume henceforth that $C$ is a cofibrant $\mathcal{A}_{\infty}$-operad.

The proof that a cofibrant operad suffices is quite simple, so we sketch it here (using notions from Section 22.2). Let $Y$ be a cofibrant $C$-algebra over a cofibrant operad $C$. It will suffice to can produce a $C$-algebra $X$ for which $U X$ is cofibrant as a spectrum together with a trivial fibration $p: X \rightarrow Y$ of $C$-algebras, for then the theory of model categories tells us that $p$ admits a section, and so $U Y$ is a retract of the cofibrant spectrum $U X$ and thus cofibrant.

We produce such an $X$ by means of a dirty trick. Start with a trivial fibration of spectra $p: X \rightarrow U Y$ in which $X$ is a cofibrant spectrum. We need to put a $C$-algebra structure on $X$ so that $p$ becomes a map of $C$-algebras. Consider the diagram

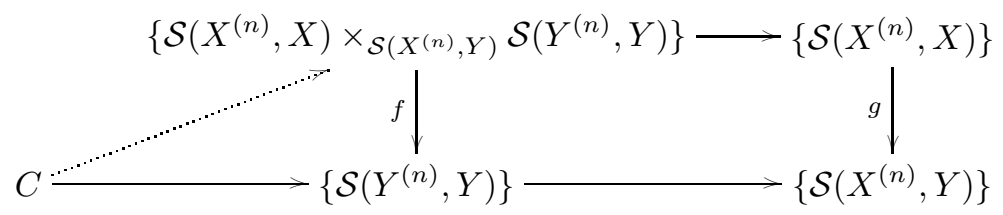

The map $g$ is bunch of trivial fibrations of spaces (one for each $n \geq 0$ ), because $X^{(n)}$ is a cofibrant spectrum and $p$ is a trivial fibration of spectra. One can show that the pull-back $f$ is actually a map between operads; since $f$ is a trivial fibration space-wise, it is a trivial fibration of operads, and the cofibrancy property of $C$ implies that the dotted arrow exists as a map of operads. You can check that such a dotted arrow gives precisely a $C$-algebra structure on $X$ making $p$ into a map of $C$-algebras, as desired!

\section{Homotopy spectral sequence}

We describe the Bousfield-Kan homotopy spectral sequence of a pointed cosimplicial space, and the Bousfield obstruction theory for an unpointed cosimplicial space.

14.1. Spectral sequence. A functor $\Delta \rightarrow \mathrm{C}$ is called a cosimplicial object in $\mathbf{C}$. Thus

$$
[n] \mapsto Y^{n}=C-\operatorname{alg}\left(C^{n+1} X, E\right)
$$

is a cosimplicial space $Y^{\bullet}$.

If $X_{\bullet}$ is a simplicial object in some category, there is a "skeletal" filtration

$$
X_{0} \simeq\left|X_{\bullet}\right|_{0} \rightarrow\left|X_{\bullet}\right|_{1} \rightarrow\left|X_{\bullet}\right|_{2} \rightarrow \cdots \rightarrow\left|X_{\bullet}\right|
$$


where $\left|X_{\bullet}\right|_{k}$ is a quotient of $\coprod_{0 \leq n \leq k} \Delta_{n} \otimes X_{n}$. This gives a tower

$$
C-\operatorname{alg}\left(\left|X_{\bullet}\right|_{0}, E\right) \leftarrow C-\operatorname{alg}\left(\left|X_{\bullet}\right|_{1}, E\right) \leftarrow \cdots \leftarrow C-\operatorname{alg}\left(\left|X_{\bullet}\right|, E\right)
$$

of spaces. If $X_{\bullet}$ is "Reedy cofibrant", then the induced tower is a tower of fibrations.

The spaces in the tower, called the Tot-tower, can be derived directly from the cosimplicial space $Y^{\bullet}$. The total space $\operatorname{Tot}\left(Y^{\bullet}\right)$ is a subspace of $\prod_{n \geq 0} \operatorname{map}\left(\Delta_{n}, Y^{n}\right)$. The $k$ th approximation to the total space $\operatorname{Tot}^{k}\left(Y^{\bullet}\right)$ is the corresponding subspace of $\prod_{0 \leq n \leq k} \operatorname{map}\left(\Delta_{n}, Y^{n}\right)$. One has

$$
C-\operatorname{alg}\left(\left|C^{\bullet+1}\right|_{k}, E\right) \simeq \operatorname{Tot}^{k} Y^{\bullet} .
$$

(The Reedy cofibrancy condition on $X_{\bullet}$ is the exact condition needed for the cosimplicial space $Y^{\bullet}$ to be fibrant in the sense of Bousfield and Kan [3].)

Suppose we have chosen a $C$-algebra map $f: X \rightarrow E$. This gives us base-points $C^{n+1} X \rightarrow X \rightarrow E$ in $Y^{n}$, and a base-point in Tot $Y^{\bullet}$. The Bousfield-Kan spectral sequence of a pointed cosimplicial space $[\mathbf{3}]$ is a second quadrant spectral sequence "converging" to $\pi_{*}\left(\operatorname{Tot} Y^{\bullet}, f\right)$, where

$$
E_{2}^{s, t}=\pi^{s} \pi_{t}\left(Y^{\bullet}, f\right) \Longrightarrow \pi_{t-s}\left(\operatorname{Tot} Y^{\bullet}, f\right) .
$$

There are differentials

$$
d_{r}: E_{r}^{s, t} \rightarrow E_{r}^{s+r, t+r-1} .
$$

Some comment on the $E_{2}$-term is appropriate. $E_{2}^{*, t}$ is the "cohomotopy" of the cosimplicial object

$$
\pi_{t} Y^{0} \rightleftharpoons \pi_{t} Y^{1} \Longleftarrow \pi_{t} Y^{2} \stackrel{\Longleftarrow}{\Longleftarrow} \cdots
$$

where homotopy groups are taken at the base-point determined by $f$. If $t \geq 2$, then the cohomotopy of the cosimplicial object is just the cohomology of the corresponding co-chain complex. The same also holds for $t=1$ if all $Y^{s}$ are look spaces. The "corner" $E_{2}^{0,0}$ is only a pointed set, defined as the equalizer

$$
E_{2}^{0,0}=\operatorname{Ker}\left(\pi_{0} Y^{0} \rightrightarrows \pi_{0} Y^{1}\right) \text {. }
$$

14.2. Obstruction theory. The above spectral sequence can be defined once we have chosen a point in Tot $Y^{\bullet}$. But what if we don't know whether such a point exists (this is one of the things we want to prove). There is an obstruction theory, due to Bousfield [2], which lets us solve this problem.

First, suppose we choose a point $y_{0} \in \operatorname{Tot}^{0} Y^{\bullet} \simeq Y^{0}$. This defines a class $\left[y_{0}\right] \in \pi_{0} Y^{0}$, which we can think of as $E_{1}^{0,0}$. We can lift $y_{0}$ to $\operatorname{Tot}^{1} Y^{\bullet}$ if and only if $\left[y_{0}\right]$ is in the equalizer of $\pi_{0} Y^{0} \rightarrow \pi_{0} Y^{0}$. That is, $y_{0}$ lifts only if the class $\left[y_{0}\right]$ "survives" to $E_{2}^{0,0}$.

The next step is to lift to $\operatorname{Tot}^{2} Y^{\bullet}$. The problem here involves extending a map from $\partial \Delta_{2} \rightarrow Y^{2}$ to all of $\Delta_{2}$; it turns out that if a point $y_{0} \in \operatorname{Tot}^{0} Y^{\bullet}$ can be lifted to $\operatorname{Tot}^{1} Y^{\bullet}$, then the obstruction to lifting $y_{0}$ to $\operatorname{Tot}^{2} Y^{\bullet}$ lies in $E_{2}^{2,1}$ (at least if all the $\pi_{1} Y^{s}$ act trivially on $\pi_{t} Y^{s}$, so that this group is well defined; there is a description of this obstruction even when this is not true, but then the obstruction is just a relation, rather than a map).

This process continues; to lift to $\operatorname{Tot}^{3} Y^{\bullet}$ we have to show that an obstruction in $E_{3}^{3,2}$ vanishes. The result we need is 
THEOREM 14.3 (Bousfield [2, 6.1]). If $Y^{\bullet}$ is a cosimplicial space, and given a vertex $y_{0} \in Y^{0}$ such that $\left[y_{0}\right] \in \operatorname{Ker}\left(\pi_{0} Y^{0} \rightrightarrows \pi_{0} Y^{1}\right)$, and if

$$
E_{2}^{s, s-1}=0
$$

for $s \geq 2$, then $y_{0}$ lifts to $\operatorname{Tot} Y^{\bullet}$.

14.4. Computing spaces of maps of $\mathcal{A}_{\infty}$ algebras. Let $E$ and $F$ be $\mathcal{A}_{\infty^{-}}$ ring spectra (over our chosen $\mathcal{A}_{\infty}$-operad $C$ ), such that the underlying spectra of $E$ and $F$ are equivalent to $E_{k_{1}, \Gamma_{1}}$ and $E_{k_{2}, \Gamma_{2}}$ respectively. Suppose furthermore that $F$ is cofibrant as a $C$-algebra spectrum. Then we can construct a simplicial resolution of $F$, and thereby get an obstruction theory and potentially a spectral sequence computing the homotopy of $C-\operatorname{alg}(F, E)$.

THEOREM 14.5. In the above situation

$$
\begin{gathered}
E_{2}^{0,0} \simeq \operatorname{Hom}_{E_{*}-\text { alg }}\left(E_{*} F, E_{*}\right), \\
E_{2}^{s, t} \simeq \operatorname{Der}_{E_{*}-\operatorname{alg}}^{s}\left(E_{*} F, E_{*+t}\right), \quad \text { for } t-s \geq-1, t>0 . \\
\operatorname{Hom}_{E_{*}-\operatorname{alg}}\left(E_{*} F, E_{*}\right) \simeq \mathcal{F} \mathcal{G}\left(\left(k_{1}, \Gamma_{1}\right),\left(k_{2}, \Gamma_{2}\right)\right), \\
\operatorname{Der}_{E_{*}-\operatorname{alg}}^{s}\left(E_{*} F, E_{*+t}\right) \simeq 0, \quad \text { for all } s, t \geq 0 .
\end{gathered}
$$

Proof. Part (1a) is proved in Section 16. Part (1b) is proved in Section 18. Part (2a) is proved in Section 17. Part (2b) is proved in Sections 19, 20, and 21.

COROLlary 14.6. The topological functor

$$
\mathcal{A}_{\infty}^{\mathrm{LT}} \rightarrow \mathcal{F} \mathcal{G}^{\mathrm{op}}
$$

is fully faithful (i.e., induces weak equivalence on mapping spaces for all objects of $\mathcal{A}_{\infty}^{\mathrm{LT}}$.)

Proof. Given objects $E, F \in \mathcal{A}_{\infty}^{\mathrm{LT}}$ which map to formal group laws $\left(k_{1}, \Gamma_{1}\right)$ and $\left(k_{2}, \Gamma_{2}\right)$ respectively, we see by Theorem 14.3 and Theorem 14.5 that $\mathcal{A}_{\infty}(F, E)$ is a homotopy discrete space with $\pi_{0} \mathcal{A}_{\infty}(F, E) \simeq \mathcal{F} \mathcal{G}\left(\left(k_{1}, \Gamma_{1}\right),\left(k_{2}, \Gamma_{2}\right)\right)$.

\section{Part 3. Calculations}

In the following sections we compute the obstruction groups and $E_{2}$-term of the spectral sequence described in Section 14. These calculations will also be used in Section 23 when we construct $\mathcal{A}_{\infty}$-structures.

\section{Preliminary calculations}

Let $E$ and $F$ be Landweber exact spectra. Then

$$
E_{*} F \simeq E_{*} \underset{M U_{*}}{\otimes} M U_{*} M U \underset{M U_{*}}{\otimes} F_{*} .
$$

Proposition 15.1. For Landweber exact theories $E_{*}$ and $F_{*}$, the module $E_{*} F$ is flat over $E_{*}$. 
Proof. It suffices to show that $E_{*} M U$ is flat as an $M U_{*}$ module. That is, we must show that the functor

$$
\left\{M U_{*} \text {-modules }\right\} \longrightarrow\left\{E_{*} \text {-modules }\right\}
$$

defined by $N \mapsto E_{*} M U \underset{M U_{*}}{\otimes} N$ is exact. But this functor factors

$$
\left\{M U_{*} \text {-modules }\right\} \longrightarrow\left\{M U_{*} M U \text {-comodules }\right\} \longrightarrow\left\{E_{*} \text {-modules }\right\},
$$

sending

$$
N \mapsto M U_{*} M U \underset{M U_{*}}{\otimes} N \mapsto E_{*} \underset{M U_{*}}{\otimes} M U_{*} M U \underset{M U_{*}}{\otimes} N \simeq E_{*} M U \underset{M U_{*}}{\otimes} N,
$$

where the left-hand functor is exact since $M U_{*} M U$ is a flat module over $M U_{*}$, and the right-hand functor is exact by Landweber's theorem.

15.2. Universal coefficient and Künneth theorems. Let $E$ be a ringspectrum, and $X$ and $Y$ arbitrary spectra. One would like to have a Künneth spectral sequence

$$
\operatorname{Tor}_{s}^{E_{*}}\left(E_{*} X, E_{*+t} Y\right) \Longrightarrow E_{s+t}(X \wedge Y)
$$

and a coefficient spectral sequence

$$
\operatorname{Ext}_{E_{*}}^{s}\left(E_{*} X, E_{*+t}\right) \Longrightarrow E^{t-s} X .
$$

This doesn't happen in general, but it does happen in many cases for good spectra E. Adams [1, part 3] gave a general criterion for the existence of such spectral sequences. We need Adams' criterion in one case; it is a nice observation of Hopkins.

Proposition 15.3. If $E$ is a Landweber exact theory, then there are Künneth and universal coefficient spectral sequences as described above.

Proof. Adams constructs the spectral sequences for $E$ using the fact that for any arbitrary spectrum $X$ there exists a map $P \rightarrow X$ of spectra such that

(a) $E_{*} P \rightarrow E_{*} X$ is surjective,

(b) $E_{*} P$ is projective over $E_{*}$,

(c) $E_{*}(P \wedge Y) \simeq E_{*} P \underset{E_{*}}{\otimes} E_{*} Y$ (for the Künneth theorem), and

(d) $E^{*} P \simeq \operatorname{Hom}_{E_{*}}\left(E_{*} P, E_{*}\right)$ (for the coefficient theorem).

In the case when $E=M U$, Adams shows that it suffices to take $P$ to be an appropriate wedge of Spanier-Whitehead duals of Thom spectra of finite complex Grassmanians.

If $P \rightarrow X$ is the map Adams constructed for $M U$, and $E$ is a Landweber exact theory, then properties (a), (b), (c), and (d) hold for $E$ : (a) and (b) follow because tensoring preserves surjections and projectives, (c) is trivial, and (d) follows because $P$ is a wedge of finite spectra $T$, and because $E^{*} T \simeq E_{*} D T$ is a free module over $E_{*}$ by computation, since $D T$ is a Thom spectrum of a finite complex Grassmanian.

COROLlaRY 15.4. If $E$ and $F_{i}(i=1, \ldots, n)$ are Landweber exact spectra, and $F \simeq F_{1} \wedge \cdots \wedge F_{n}$, then $E_{*} F$ is flat over $E_{*}$, and

$$
E_{*} F \simeq E_{*} F_{1} \underset{E_{*}}{\otimes} \cdots \underset{E_{*}}{\otimes} E_{*} F_{n} .
$$

Proposition 15.5. If $E=E_{k_{1}, \Gamma_{1}}$ and $F=E_{k_{2}, \Gamma_{2}}$, then the evaluation map

$$
E^{*} F \rightarrow \operatorname{Hom}_{E_{*}}\left(E_{*} F, E_{*}\right)
$$

is an isomorphism. 
Proof. By the universal coefficient theorem, we only need to prove that the $\mathrm{Ext}^{s}$ groups vanish for $s>0$. This follows from Lemma 15.6.

Lemma 15.6. Let $M$ be a flat $E_{*}$-module. Then

$$
\operatorname{Ext}_{E_{*}}^{s}\left(M, E_{*}\right) \simeq 0
$$

for $s>0$.

Proof. Let $\mathfrak{m}=\left(p, u_{1}, \ldots, u_{n-1}\right)$ denote the maximal ideal of $E_{*}$. We first prove that $\operatorname{Ext}_{E_{*}}^{s}\left(M, E_{*} / \mathfrak{m}^{d}\right) \simeq 0$ for each $d$.

For $d=1$ we have

$$
\operatorname{Ext}_{E_{*}}^{s}\left(M, E_{*} / \mathfrak{m}\right) \simeq \operatorname{Ext}_{E_{*} / \mathfrak{m}}^{s}\left(M \underset{E_{*}}{\otimes} E_{*} / \mathfrak{m}, E_{*} / \mathfrak{m}\right)
$$

by flat base change. Since $E_{*} / \mathfrak{m} \simeq k\left[u^{ \pm}\right]$is injective as a graded module over itself, these Ext groups are 0 for $s>0$.

Note that $\mathfrak{m}^{d} / \mathfrak{m}^{d+1}$ is a finite $k\left[u^{ \pm}\right]$-vector space, so $\operatorname{Ext}_{E_{*}}^{s}\left(M, \mathfrak{m}^{d} / \mathfrak{m}^{d+1}\right) \simeq 0$ for $s>0$. Thus for $d>1$, the exact sequence

$$
0 \rightarrow \mathfrak{m}^{d} / \mathfrak{m}^{d+1} \rightarrow E_{*} / \mathfrak{m}^{d+1} \rightarrow E_{*} / \mathfrak{m}^{d} \rightarrow 0
$$

shows that $\operatorname{Ext}_{E_{*}}^{s}\left(M, E_{*} / \mathfrak{m}^{d+1}\right)=0$ for $s>0$, as well as showing that the map $\operatorname{Hom}_{E_{*}}\left(M, E_{*} / \mathfrak{m}^{d+1}\right) \rightarrow \operatorname{Hom}_{E_{*}}\left(M, E_{*} / \mathfrak{m}^{d}\right)$ is surjective.

Finally, use the exact sequence

$$
0 \rightarrow E_{*} \rightarrow \prod E_{*} / \mathfrak{m}^{d} \stackrel{1-S}{\longrightarrow} \prod E_{*} / \mathfrak{m}^{d} \rightarrow 0
$$

in $\operatorname{Ext}_{E_{*}}^{s}(M,-)$ to prove the lemma. (Here $S$ denotes the "shift" map.)

REMARK 15.7. Note that Lemma 15.6 and hence Proposition 15.5 (and, as we shall see, Lemma 18.4 and Corollary 19.3) can fail for an arbitrary Landweber exact theory $E_{*}$. For example, if $E=E(n)$, the Johnson-Wilson theory with $E(n)_{*} \simeq$ $\mathbb{Z}_{(p)}\left[v_{1}, \ldots, v_{n-1}, v_{n}^{ \pm}\right]$, then it is not the case that $\operatorname{Ext}_{E_{*}}^{1}\left(E_{*} E, E_{*}\right)$ vanishes, even though $E_{*} E$ is flat over $E_{*}$.

\section{Identification of $E_{2}^{0,0}$}

In this section we show that

$$
E_{2}^{0,0} \simeq \operatorname{Hom}_{E_{*}-\operatorname{alg}}\left(E_{*} F, E_{*}\right),
$$

proving part (1a) of Theorem 14.5

We need to compute the equalizer of the pair of maps

$$
\pi_{0} C-\operatorname{alg}(C F, E) \rightrightarrows \pi_{0} C-\operatorname{alg}\left(C^{2} F, E\right) .
$$

First note that for any $C$-algebra $Y$ there is a natural map

$$
\pi_{0} C-\operatorname{alg}(Y, E) \longrightarrow \operatorname{Hom}_{E_{*}-\operatorname{alg}}\left(E_{*} Y, E_{*}\right) .
$$

This map sends a $C$-algebra map $f: Y \rightarrow E$ to the map

$$
E_{*} Y \stackrel{E_{*} f}{\longrightarrow} E_{*} E \rightarrow E_{*} .
$$

Note that $E_{*} Y$ is in fact an associative $E_{*}$ algebra, since the $\mathcal{A}_{\infty}$-structure on $Y$ induces maps $E_{*} Y \underset{E_{*}}{\otimes} E_{*} Y \rightarrow E_{*}(Y \wedge Y) \rightarrow E_{*} Y$. 
Now suppose $Y=C X$ for some spectrum $X$. Given an $E_{*}$ module $M$, let $T M$ denote the $E_{*}$ tensor algebra on $M$; i.e.,

$$
T M=\bigoplus_{n \geq 0} M^{\otimes n} \simeq E_{*} \oplus M \oplus\left(M \underset{E_{*}}{\otimes} M\right) \oplus \cdots .
$$

At the level of the homotopy category of spectra,

$$
Y=C X \simeq \bigvee_{n \geq 0} X^{(n)} \simeq S^{0} \vee X \vee X^{(2)} \vee \cdots
$$

The inclusion $X \rightarrow C X$ induces a map $E_{*} X \rightarrow E_{*} C X$ of $E_{*}$-modules, and hence a map $\alpha_{X}: T\left(E_{*} X\right) \rightarrow E_{*} C X$ of algebras.

Lemma 16.1. If $E_{*} X$ is flat over $E_{*}$, then $\alpha_{X}$ is an isomorphism.

Proof. This is clear from Corollary 15.4.

In particular Lemma 16.1 holds for $X=C^{n} F$.

Now consider the commutative diagram

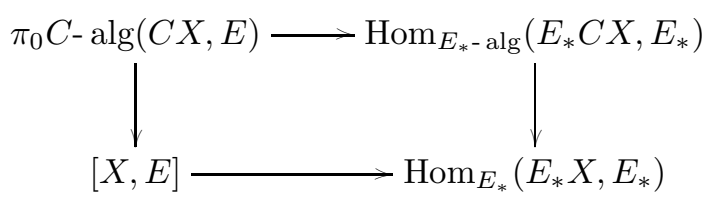

The left-hand vertical arrow is an isomorphism, since $C X$ is the free $C$-algebra on $X$. If $E_{*} X$ is flat over $E_{*}$, the bottom and right-hand vertical arrows are isomorphisms by Proposition 15.5 and Lemma 16.1; hence the top arrow is also an isomorphism. Thus, we have reduced our problem to that of computing the equalizer of the pair of maps

$$
\operatorname{Hom}_{E_{*} \text { alg }}\left(T\left(E_{*} F\right), E_{*}\right) \rightrightarrows \operatorname{Hom}_{E_{*} \text { alg }}\left(T^{2}\left(E_{*} F\right), E_{*}\right) \text {. }
$$

But this is precisely just the equalizer of

$$
\operatorname{Hom}_{E_{*}}\left(E_{*} F, E_{*}\right) \rightrightarrows \operatorname{Hom}_{E_{*}}\left(T\left(E_{*} F\right), E_{*}\right)
$$

where the top arrow sends $f: E_{*} F \rightarrow E_{*}$ to the composite

$$
T\left(E_{*} F\right) \rightarrow E_{*} F \stackrel{f}{\rightarrow} E_{*}
$$

and the bottom arrow sends it to the composite

$$
T\left(E_{*} F\right) \stackrel{T(f)}{\longrightarrow} T\left(E_{*}\right) \rightarrow E_{*} .
$$

Thus

$$
E_{2}^{0,0}=\operatorname{Hom}_{E_{*-} \operatorname{alg}}\left(E_{*} F, E_{*}\right) .
$$

\section{Computation of $\operatorname{Hom}_{E_{*}-\operatorname{alg}}\left(E_{*} F, E_{*}\right)$}

In this section we show that

$$
\operatorname{Hom}_{E_{*} \text { alg }}\left(E_{*} F, E_{*}\right) \simeq \mathcal{F G}\left(\left(k_{1}, \Gamma_{1}\right),\left(k_{2}, \Gamma_{2}\right)\right),
$$

which proves part (2a) of Theorem 14.5.

Let $E=E_{k_{1}, \Gamma_{1}}, F=E_{k_{2}, \Gamma_{2}}$. There are universal deformations $\left(G_{e}, i_{e}\right)$ of the formal groups $\left(k_{e}, \Gamma_{e}\right)$, where $G_{e}$ is a degree 0 formal group law over the ring

$$
E\left(k_{e}, \Gamma_{e}\right)=\pi_{0} E_{k_{e}, \Gamma_{e}} \simeq \mathbb{W} k \llbracket u_{1}, \ldots, u_{n-1} \rrbracket .
$$


Recall from Section 6 that chosen invertible elements $u_{G_{e}} \in \pi_{2} E_{k_{e}, \Gamma_{e}}$ allow us to produce degree -2-formal group laws $\bar{G}_{e}$.

We need to understand the set

$$
\operatorname{Hom}_{E_{*-} \operatorname{alg}}\left(E_{*} F, B_{*}\right) \text {. }
$$

Recall from Section 15 that

$$
E_{*} F \simeq E_{*} \underset{M U_{*}}{\otimes} M U_{*} M U \underset{M U_{*}}{\otimes} F_{*} .
$$

Thus by Theorem 6.2 a map $E_{*} F \rightarrow B_{*}$ of commutative graded rings corresponds precisely to the following data: a map $\phi: E_{*} \rightarrow B_{*}$, a map $\psi: F_{*} \rightarrow B_{*}$, and a strict isomorphism $\bar{g}: \psi^{*} \bar{G}_{2} \rightarrow \phi^{*} \bar{G}_{1}$ of formal group laws. Represent such data by the triple $(\phi, \bar{g}, \psi)$.

Suppose we fix once and for all an $E_{*}$-algebra structure on $B_{*}$, in other words, a map $\phi$. Then elements of the set $\operatorname{Hom}_{E_{*} \text { alg }}\left(E_{*} F, B_{*}\right)$ correspond to data $(\psi, \bar{g})$. That is,

$$
\operatorname{Hom}_{E_{*} \text { alg }}\left(E_{*} F, B_{*}\right) \simeq\left\{\left(\psi: F_{*} \rightarrow B_{*}, \bar{g}: \psi^{*} \bar{G}_{2} \stackrel{\sim}{\longrightarrow} \phi^{*} \bar{G}_{1}\right)\right\} .
$$

Write $\psi_{0}: F_{0} \rightarrow B_{0}$ for the restriction of $\psi$ to degree 0 . Since $F_{*} \simeq F_{0}\left[u_{G_{2}}^{ \pm}\right]$, the set of homomorphisms $\psi: F_{*} \rightarrow B_{*}$ corresponds bijectively to the set of pairs $\left(\psi_{0}, \psi\left(u_{G_{2}}\right)\right)$ where $\psi_{0}$ is a map $F_{0} \rightarrow B_{0}$ and $\psi\left(u_{G_{2}}\right)$ is an invertible element in $B_{2}$.

Recall from Section 6 that the strict isomorphism $\bar{g}: \psi^{*} \bar{G}_{2} \rightarrow \phi^{*} \bar{G}_{1}$ of degree -2 formal group laws and the elements $\phi\left(u_{G_{1}}\right)$ and $\psi\left(u_{G_{2}}\right)$ induce an isomorphism $g: \psi_{0}^{*} G_{2} \rightarrow \phi_{0}^{*} G_{1}$ of degree 0 formal group laws, not necessarily strict, by $g(x)=$ $\phi\left(u_{G_{1}}\right) \bar{g}\left(\psi\left(u_{G_{2}}\right)^{-1} x\right)$. Note that if $\bar{g}(x)=x+a_{2} x^{2}+a_{3} x^{3}+\cdots$, then

$$
g(x)=\left(\phi\left(u_{G_{1}}\right) \psi\left(u_{G_{2}}\right)^{-1}\right) x+a_{2}\left(\phi\left(u_{G_{1}}\right) \psi\left(u_{G_{2}}\right)^{-2}\right) x^{2}+\cdots .
$$

Thus, given $g(x)$ and $\phi\left(u_{G_{1}}\right)$, we can recover $\psi\left(u_{G_{2}}\right)$ (since $\phi\left(u_{G_{1}}\right)=g^{\prime}(0) \psi\left(u_{G_{2}}\right)$ ), and hence recover $\bar{g}(x)$.

Putting this together, we see that the data $(\psi, \bar{g})$ corresponds bijectively to data $\left(\psi_{0}, g\right)$. Thus, we have proved

Proposition 17.1. There is an isomorphism of sets

$$
\operatorname{Hom}_{E_{*}-\operatorname{alg}}\left(E_{*} F, B_{*}\right) \simeq\left\{\left(\psi_{0}: F_{0} \rightarrow B_{0}, g: \psi^{*} G_{2} \stackrel{\sim}{\longrightarrow} \phi^{*} G_{1}\right)\right\} .
$$

REMARK 17.2. There is another way to describe this set. It is easy to see that $E_{*} F \simeq E_{0} F\left[u_{G_{1}}\right]$, so that

$$
\operatorname{Hom}_{E_{*}-\operatorname{alg}}\left(E_{*} F, B_{*}\right) \simeq \operatorname{Hom}_{E_{0} \text {-alg }}\left(E_{0} F, B_{0}\right) .
$$

Thus, a map from $E_{0} F$ to a ring $B$ corresponds precisely to: a map $\phi: E_{0} \rightarrow B$, a map $\psi: F_{0} \rightarrow B$, and an isomorphism $g: \psi^{*} G_{2} \stackrel{\sim}{\longrightarrow} \phi^{*} G_{1}$ (not necessarily strict).

Equivalently, there is a square diagram of groupoids

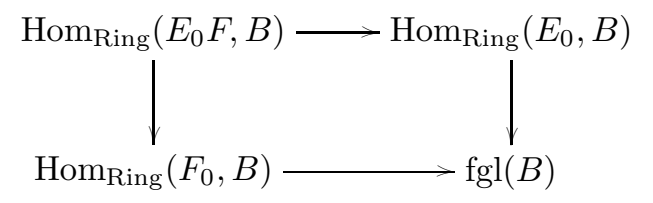

commuting up to a natural isomorphism, which is a homotopy pull-back square. (A set $S$ is regarded as a groupoid having as objects the set $S$, and as maps only identity maps.) This way of viewing things will come in handy in Section 21. 
Suppose now that $B=B_{0}$ is a complete local ring, and that $B$ is a continuous $E_{0}$-algebra, i.e., the map $\phi_{0}: E_{0} \rightarrow B$ is a continuous map between complete local rings. The continuity hypothesis is equivalent to requiring that $\phi_{0}\left(\mathfrak{m}_{E}\right) \subset \mathfrak{m}_{B}$, where $\mathfrak{m}_{E}=\left(p, u_{1}, \ldots, u_{n-1}\right) \subset E_{0}$ is the maximal ideal. Given some $E_{0}$-algebra map $E_{0} F \rightarrow B$, I would like to know that the induced map $F_{0} \rightarrow E_{0} F \rightarrow B$ between complete rings is continuous. This is ensured by the following well-known lemma.

Lemma 17.3. Let $\mathfrak{m}_{E} \subset E_{0}$ and $\mathfrak{m}_{F} \subset F_{0}$ be the maximal ideals of these rings. Then $\mathfrak{m}_{E} E_{0} F=\mathfrak{m}_{F} E_{0} F$ as ideals of $E_{0} F$.

Proof. The ideal $\mathfrak{m}_{E}$ is generated by the coefficients of the terms of the $p$ series $[p]_{G_{1}}(x)$ which are of degree less than $p^{n}$. Likewise, the ideal $\mathfrak{m}_{F}$ is generated by the coefficients of the terms of the $p$-series $[p]_{G_{2}}(x)$ which are of degree less than $p^{n}$. There is an isomorphism of formal group laws $g: G_{1} \rightarrow G_{2}$ defined over the ring $E_{0} F$, and hence an equation $g \circ[p]_{G_{1}}=[p]_{G_{2}} \circ g$. The lemma follows easily.

We have fixed a map $\phi_{0}: E_{0} \rightarrow B$ and hence a formal group law $\phi^{*} G_{1}$ over $B$ which is a deformation of $\left(k_{1}, \Gamma_{1}\right)$ to $B$. Maps $\psi: F_{0} \rightarrow B$ classify $\star$-isomorphism classes of deformations of $\left(k_{2}, \Gamma_{2}\right)$ to $B$. Consider the diagram of groupoids

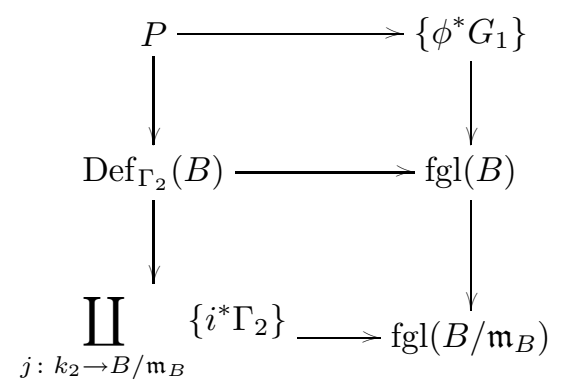

in which both squares are homotopy-pullback squares of groupoids. We get two descriptions of $P$ from this diagram. The first comes from the top square: since $\operatorname{Def}_{\Gamma_{2}}(B)$ is homotopy discrete and $\pi_{0} \operatorname{Def}_{\Gamma_{2}}(B) \simeq \operatorname{Hom}_{\text {Ring }}^{\text {cts }}\left(F_{0}, B\right)$, we see that $P$ is equivalent to a disjoint union of a bunch of loop-spaces of $\operatorname{fgl}(B)$, and in fact $P$ is homotopy discrete with

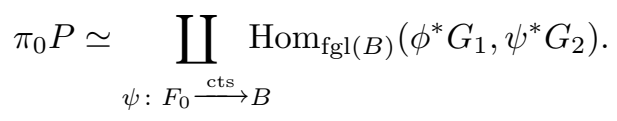

Thus, $P \simeq \operatorname{Hom}_{E_{*} \text { alg }}\left(E_{*} F, B_{*}\right)$ by Proposition 17.1 and Lemma 17.3.

The second description comes from the big rectangle. This also describes $P$ as a union of loop-spaces, and shows that $P$ is homotopy discrete with

$$
\pi_{0} P \simeq \coprod_{j: k_{2} \rightarrow B / \mathfrak{m}_{B}} \operatorname{Hom}_{\mathrm{fgl}\left(B / \mathfrak{m}_{B}\right)}\left(i^{*} \Gamma_{1}, j^{*} \Gamma_{2}\right),
$$

where $i: k_{1} \rightarrow B / \mathfrak{m}_{B}$ is the map induced by $\phi_{0}: E_{0} \rightarrow B$ modulo maximal ideals. Thus we conclude

$$
\operatorname{Hom}_{E_{*}-\operatorname{alg}}\left(E_{*} F, B_{*}\right) \simeq P \simeq \mathcal{F G}\left(\left(B / \mathfrak{m}_{B}, i^{*} \Gamma_{1}\right),\left(k_{2}, \Gamma_{2}\right)\right) .
$$

If we let $B_{*}=E_{*}$ with $\phi=\mathrm{id}$, then we get an isomorphism

$$
\operatorname{Hom}_{E_{*}-\operatorname{alg}}\left(E_{*} F, E_{*}\right) \simeq \mathcal{F G}\left(\left(k_{1}, \Gamma\right),\left(k_{2}, \Gamma\right)\right)
$$


as desired.

REMARK 17.4. Note that if we let $B_{*}=E_{*} / \mathfrak{m}^{d}$ for any $d \geq 1$ we still get an isomorphism

$$
\operatorname{Hom}_{E_{*}-\operatorname{alg}}\left(E_{*} F, E_{*} / \mathfrak{m}^{d}\right) \simeq \mathcal{F} \mathcal{G}\left(\left(k_{1}, \Gamma_{1}\right),\left(k_{2}, \Gamma_{2}\right)\right) .
$$

In particular, this holds for $d=1$. If we restrict to degree 0 and then tensor down via the map $E_{0} \rightarrow E_{0} / \mathfrak{m}=k_{1}$, we see that

$$
\operatorname{Hom}_{k_{1}-\operatorname{alg}}\left(E_{0} F / \mathfrak{m}, k_{1}\right) \simeq \mathcal{F G}\left(\left(k_{1}, \Gamma_{1}\right),\left(k_{2}, \Gamma_{2}\right)\right)
$$

where $\mathfrak{m}$ denotes the ideal generated by $p, u_{1}, \ldots, u_{n-1} \in E_{0} \subset E_{0} F$. This means one can give a description of $E_{0} F / \mathfrak{m}$ in many cases using formal group law theory.

For example, if $\Gamma_{1}=\Gamma_{2}=\Gamma$ is the Honda formal group law, so that $E=$ $F=E_{n}$, then $\mathcal{F G}\left(\left(\mathbb{F}_{p^{n}}, \Gamma\right),\left(\mathbb{F}_{p^{n}}, \Gamma\right)\right)$ is isomorphic to the set of pairs $(\sigma, f)$ where $\sigma: \mathbb{F}_{p^{n}} \rightarrow \mathbb{F}_{p^{n}}$ is a Galois automorphism, and $f(x)=e_{0} x+e_{\Gamma} x_{\Gamma}^{p}+e_{2} x^{p^{2}}+\cdots$ is a power series with coefficients $e_{i} \in \mathbb{F}_{p^{n}}$, with $e_{0} \neq 0$ (see [13, App. 2]). This shows that

$$
E_{0} F / \mathfrak{m} \simeq\left(\mathbb{F}_{p^{n}} \underset{\mathbb{F}_{p}}{\otimes} \mathbb{F}_{p^{n}}\right)\left[e_{0}, e_{1}, e_{2}, \ldots\right] /\left(e_{0}^{p^{n}-1}-1, e_{1}^{p^{n}}-e_{1}, e_{2}^{p^{n}}-e_{2}, \ldots\right)
$$

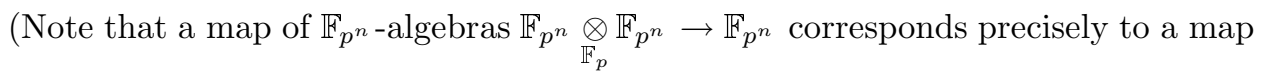
of rings $\sigma: \mathbb{F}_{p^{n}} \rightarrow \mathbb{F}_{p^{n}}$.)

\section{Derivations of associative algebras}

In this section we define the derived functors of associative algebra derivations and show part (1b) of Theorem 14.5.

18.1. A complex for the $E_{2}$-term. Suppose $Y$ is a $C$-algebra, and choose a map $f: Y \rightarrow E$. The set $\pi_{t}(C-\operatorname{alg}(Y, E), f)$ is the same as homotopy classes of maps $S^{t} \rightarrow C$ - $\operatorname{alg}(Y, E)$ which send the base-point of the sphere to $f$, and where homotopies are base-point preserving.

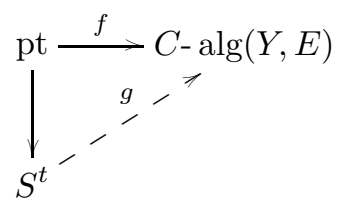

By adjunction, these are the same as homotopy classes of lifts in the diagram

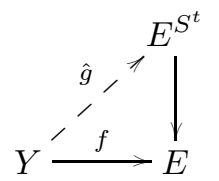

of $C$-algebras, where homotopies are ones which are fiberwise over $f$. Recall from Section 12.2 that $E^{S^{t}}$ is a $C$-algebra whose underlying spectrum is isomorphic to $F\left(\Sigma^{\infty} S_{+}^{t}, E\right) \simeq E \vee \Sigma^{-t} E$. Thus $\pi_{*} E^{S^{t}} \simeq E^{-*} S^{t} \simeq E_{*}[\epsilon] / \epsilon^{2}$, with $|\epsilon|=-t$. The 
spectrum $E^{S^{t}}$ is naturally a ring spectrum over $E$. Applying $E_{*}(-)$ everywhere and using the ring structure of $E_{*}$ gives us a diagram of algebras

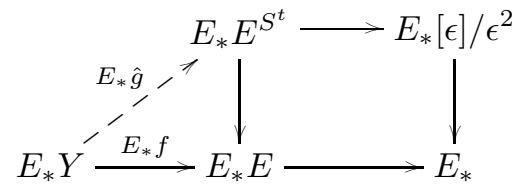

and thus we get a map

$$
\pi_{t}(C-\operatorname{alg}(Y, E), f) \rightarrow \operatorname{Hom}_{\left(E_{*}-\operatorname{alg} \downarrow E_{*}\right)}\left(E_{*} Y, E_{*}\left[\epsilon_{t}\right] / \epsilon_{t}^{2}\right),
$$

where the right hand side denotes maps in the category of $E_{*}$-algebras augmented over $E_{*}$. Note that $E_{*} Y$ is augmented over $E_{*}$, via the composite $E_{*} Y \rightarrow E_{*} E \rightarrow$ $E_{*}$; this augmentation itself depends on the choice of $f: Y \rightarrow E$.

Now suppose $Y=C X$ where $E_{*} X$ is a flat $E_{*}$-module. Then using the commutative diagram

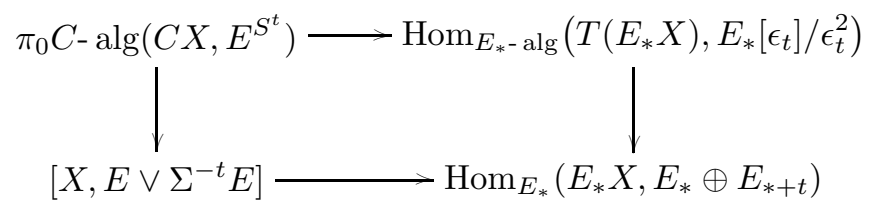

and an argument similar to that of Section 16 one shows that the top horizontal map is a bijection. Thus, we have

$$
E_{2}^{s, t} \simeq H^{s}\left[\operatorname{Hom}_{\left(E_{*}-\operatorname{alg} \downarrow E_{*}\right)}\left(T^{\bullet+1}\left(E_{*} F\right), E_{*}\left[\epsilon_{t}\right] / \epsilon_{t}^{2}\right)\right],
$$

for $t>0$ and $t-s \geq-1$.

18.2. Associative algebra derivations. Let $A$ be a commutative ring, and suppose $R$ is an associative $A$-algebra. Let $M$ be an $R$-bimodule relative to $A$; that is, $M$ is a module over the $\operatorname{ring} R^{e}=R \underset{A}{\otimes} R^{\text {op }}$. Then form a $\operatorname{ring} R \oplus M$ with multiplication given by

$$
(r, m) \cdot\left(r^{\prime}, m^{\prime}\right)=\left(r r^{\prime}, r m^{\prime}+m r^{\prime}\right)
$$

for $r, r^{\prime} \in R, m, m^{\prime} \in M$. The inclusion map $\eta: R \rightarrow R \oplus M$ and the projection map $\pi: R \oplus M \rightarrow R$ are algebra maps, and $M \subset R \oplus M$ is an ideal such that $M^{2}=0$.

Consider sections $s: R \rightarrow R \oplus M$ of the projection $\pi$. Such an $s=($ id, $D)$ where $D: R \rightarrow M$ is an $A$-module map such that

$$
D\left(r r^{\prime}\right)=r\left(D r^{\prime}\right)+(D r) r^{\prime}
$$

for $r, r^{\prime} \in R$. Such a map $D: R \rightarrow M$ is called an $A$-derivation of $R$ into $M$. The set of $A$-derivations is written $\operatorname{Der}_{A}(R, M)$, whence

$$
\operatorname{Hom}_{(A-\operatorname{alg} \downarrow R)}(R, R \oplus M) \simeq \operatorname{Der}_{A}(R, M) .
$$

More generally, let $\phi: R \rightarrow S$ be a map of associative $A$-algebras, and let $M$ be an $S$-bimodule. The map $\phi$ makes $M$ into an $R$-bimodule by restriction. Hence

$$
\operatorname{Hom}_{(A-\operatorname{alg} \downarrow S)}(R, S \oplus M) \simeq \operatorname{Der}_{A}(R, M) .
$$


Note that $\operatorname{Der}_{A}(R, M)$ is naturally an $A$-module. If $A=S=E_{*}, R=E_{*} Y$, and $M=E_{*+t}$, then this module is

$$
\operatorname{Der}_{E_{*}}\left(E_{*} Y, E_{*+t}\right) \simeq \operatorname{Hom}_{\left(E_{*}-\operatorname{alg} \downarrow E_{*}\right)}\left(E_{*} Y, E_{*}\left[\epsilon_{t}\right] / \epsilon_{t}^{2}\right) .
$$

Thus,

$$
E_{2}^{s, t} \simeq H^{s}\left[\operatorname{Der}_{E_{*}}\left(T^{\bullet+1}\left(E_{*} F\right), E_{*+t}\right)\right]
$$

18.3. Derived functors of associative algebra derivations. Let $s A$-alg denote the category of simplicial associative $A$-algebras. This has a model category structure as follows. The weak equivalences $f: R_{\bullet} \rightarrow S_{\bullet}$ are maps which induce isomorphisms on homology of the associated chain-complexes. A cofibration $f$ is a retract of a free map. A free map $f: R_{\bullet} \rightarrow S_{\bullet}$ is a map which in each simplicial degree is an inclusion

$$
R_{n} \rightarrow S_{n} \simeq R_{n}\left\langle X_{n}\right\rangle,
$$

where $R\langle X\rangle$ denotes the algebra obtained from $R$ by adjoining the elements of the set $X$ as free generators, and such that the the sets $X \bullet$ are closed under the degeneracy operators of $S_{\bullet}$. (See Quillen [11].)

A cofibrant resolution of a simplicial algebra $R_{\bullet}$ is a diagram $A \stackrel{i}{\longrightarrow} P_{\bullet} \stackrel{f}{\longrightarrow}$ $R_{\bullet}$, where $i$ is a cofibration, and $f$ is a weak equivalence. There is a functorial cofibrant resolution $P_{\bullet}$ of $R$, given by $P_{n}=F^{n+1}(R)$, where $F B$ denotes the free associative $A$-algebra on the underlying set of $R$. Another resolution is the complex $Q_{n}=T^{n+1}(R)$. It is a cofibrant resolution if $R$ is a projective $A$-module; this is easy to see if $R$ is a free $A$-module, since if $X$ is a set of module generators of $R$, then the set of monomials in $X$ is a set of module generators of $T R$.

Given an associative $A$-algebra $R$ over $S$, and an $S$-bimodule $M$, define

$$
\operatorname{Der}_{A}^{s}(R, M)=H^{s}\left[\operatorname{Der}_{A}\left(P_{\bullet}, M\right)\right]
$$

the $s$-th derived functor of derivations from $B$ to $M$, where $P_{\bullet} \rightarrow R$ is a cofibrant resolution of $R$. The derived functors do not depend on the choice of cofibrant resolution.

Our complex $T^{n+1}\left(E_{*} F\right)$ of $E_{*}$-algebras is not cofibrant, since $E_{*} F$ is $n o t$ projective over $E_{*}$; nonetheless, we will show below that this complex computes derived functors of derivations.

Suppose $R=T N$, where $N$ is an $A$-module, and let $M$ be an $S$-bimodule. Then if $P_{\bullet} \rightarrow N$ is a free resolution of $N$ by a simplicial $A$-module, then $T P_{\bullet}$ is a cofibrant resolution of $R=T N$. Then, since

$$
\operatorname{Der}_{A}(T N, M) \simeq \operatorname{Hom}_{(A-\operatorname{alg} \downarrow S)}(T N, S \oplus M) \simeq \operatorname{Hom}_{A}(N, M)
$$

it is not hard to see that

$$
\operatorname{Der}_{A}^{s}(R, M) \simeq \operatorname{Ext}_{A}^{s}(N, M)
$$

Thus, Lemma 15.6 gives

LEMmA 18.4. If we take $A=S=E_{*}, M=E_{*+t}$, and $R=T N$ where $N$ is a flat $E_{*}$-module, then

$$
\operatorname{Der}_{E_{*}}^{s}\left(T N, E_{*+t}\right)=0, \quad s>0 .
$$


18.5. The $E_{2}$-term is a derived functor. Suppose $R_{\bullet}$ is a simplicial $A$ algebra, augmented over $S$, and let $M$ be an $S$-module Then there is a spectral sequence of $A$-modules

$$
E_{1}^{p, q}=\operatorname{Der}_{A}^{p}\left(R_{q}, M\right) \Longrightarrow \operatorname{Der}_{A}^{p+q}\left(R_{\bullet}, M\right)
$$

This spectral sequence is constructed by producing a double complex $P_{p, q}$ of $A$ algebras, in which each row $P_{\bullet}, q \rightarrow R_{q}$ is a cofibrant resolution of $R_{q}$, and so that the diagonal is a cofibrant resolution of $R_{\bullet}$. Such a resolution can be constructed by the functorial cofibrant resolution $P_{p, q}=F^{p+1} R_{q}$.

Now letting $A=S=E_{*}, M=E_{*+t}$ and $R_{q}=T^{q+1}\left(E_{*} F\right)$ we see from Lemma 18.4 applied to $N=E_{*} F$ that the spectral sequence at the $E_{1}$-term is

$$
E_{1}^{p, q}=\operatorname{Der}_{E_{*}}^{p}\left(T^{q+1}\left(E_{*} F\right), E_{*+t}\right)= \begin{cases}0 & \text { if } p>0 \\ \operatorname{Der}_{E_{*}}\left(T^{q+1}\left(E_{*} F\right), E_{*+t}\right) & \text { if } p=0 .\end{cases}
$$

Thus the spectral sequence is concentrated in a single column of the $E_{1}$-term, and collapses at $E_{2}$. In fact, $E_{1}^{0, *}$ is precisely the cochain complex whose cohomology we want to compute, and by the convergence of the spectral sequence, what it computes is $\operatorname{Der}_{E_{*}}^{s}\left(E_{*} F, E_{*+t}\right)$.

\section{Change of ground ring}

In this section we show that in order to prove that $\operatorname{Der}_{E_{*}}^{s}\left(E_{*} F, E_{*+t}\right)$ vanishes, it suffices to show that $\operatorname{Der}_{E_{0} / \mathfrak{m}}^{s}\left(E_{0} F / \mathfrak{m}, E_{t} / \mathfrak{m}\right)$ vanishes.

19.1. Associative algebra differentials. Given an $A$-algebra $R$, define $I_{R / A}$ to be an $R$-bimodule equipped with a $A$-derivation $d: R \rightarrow I_{R / A}$ which induces an isomorphism

$$
\operatorname{Der}_{A}(R, M) \simeq \operatorname{Hom}_{R^{e}}\left(I_{R / A}, M\right)
$$

Such an object always exists: $I_{R / A}$ can be constructed as the cokernel of $\delta$ in

$$
R \underset{A}{\otimes} R \underset{A}{\otimes} R \underset{A}{\otimes} R \stackrel{\delta}{\rightarrow} R \underset{A}{\otimes} R \underset{A}{\otimes} R \stackrel{p}{\rightarrow} I_{R / A} \rightarrow 0
$$

where

$$
\delta(a \otimes b \otimes c \otimes d)=a b \otimes c \otimes d-a \otimes b c \otimes d+a \otimes b \otimes c d .
$$

The universal derivation $d: R \rightarrow I_{R / A}$ is given by $d(r)=p(1 \otimes r \otimes 1)$. By the bar complex, we see that $I_{R / A}$ is also the kernel of $\mu$ in

$$
0 \rightarrow I_{R / A} \rightarrow R \underset{A}{\otimes} R \stackrel{\mu}{\rightarrow} R .
$$

We call $I_{R / A}$ the $R$-bimodule of associative algebra differentials of $R$.

Let $R=T N$ be the tensor algebra on an $A$-module $N$. Then derivations $D: T N \rightarrow M$ to some $R$-bimodule $M$ correspond bijectively to maps

$$
\operatorname{Hom}_{(A-\operatorname{alg} \downarrow R)}(T N, R \oplus M) \simeq \operatorname{Hom}_{A}(N, M),
$$

and hence

$$
\operatorname{Der}_{A}(T N, M) \simeq \operatorname{Hom}_{A}(N, M) .
$$

Since $I_{T N / A}$ should be an $R$-bimodule, we see that

$$
I_{T N / A} \simeq T N \underset{A}{\otimes} N \underset{A}{\otimes} T N
$$

Note that this means that $I_{R / A}$ is a free $R$-bimodule when $R$ is a free algebra. 
Suppose $R$ is an associative $A$-algebra. Define

$$
D_{R / A}=I_{P_{\bullet} / A} \underset{P_{\bullet}^{e}}{\otimes} R^{e}
$$

for some choice $P_{\bullet} \rightarrow R$ of cofibrant resolution of $R$. Thus $D_{R / A}$ is a complex of projective $R$-bimodules, and

$$
\begin{aligned}
& \operatorname{Ext}_{R^{e}}^{s}\left(D_{R / A}, M\right) \simeq H^{s}\left[\operatorname{Hom}_{R^{e}}\left(I_{P_{\bullet} / A} \underset{P_{\bullet}^{e}}{\otimes} R^{e}, M\right)\right] \\
& \simeq H^{s}\left[\operatorname{Der}_{A}\left(P_{\bullet}, M\right)\right] \simeq \operatorname{Der}_{A}^{s}(R, M) .
\end{aligned}
$$

Here "Ext" really means some kind of "hyper-Ext".

We therefore get a coefficient long exact sequence for derived functors of derivations.

Proposition 19.2. Let $0 \rightarrow M^{\prime} \rightarrow M \rightarrow M^{\prime \prime} \rightarrow 0$ be a short exact sequence of $R$-bimodules. Then there is a long exact sequence

$0 \rightarrow \operatorname{Der}_{A}\left(R, M^{\prime}\right) \rightarrow \operatorname{Der}_{A}(R, M) \rightarrow \operatorname{Der}_{A}\left(R, M^{\prime \prime}\right) \rightarrow \operatorname{Der}_{A}^{1}(R, M) \rightarrow \cdots$.

Corollary 19.3. If $\operatorname{Der}_{E_{*}}^{s}\left(E_{*} F, E_{*+t} / \mathfrak{m}\right)=0$ for all $s$, then

$$
\operatorname{Der}_{E_{*}}^{s}\left(E_{*} F, E_{*+t}\right)=0
$$

for all $s$.

Proof. The proof is similar to that of Lemma 15.6.

19.4. Change of ground ring. Suppose $A \rightarrow A^{\prime}$ is a map of commutative rings. Then given an $A$-algebra $R$ we get an induced $A^{\prime}$-algebra $R^{\prime}=R \underset{A}{\otimes} A^{\prime}$.

Proposition 19.5. If $R$ is flat as an A-module, and $M$ is an $R^{\prime}$-bimodule, then

$$
\operatorname{Der}_{A}^{s}(R, M) \simeq \operatorname{Der}_{A^{\prime}}^{s}\left(R^{\prime}, M\right) .
$$

Proof. Let $P_{\bullet} \rightarrow R$ be a cofibrant $A$-algebra resolution of $R$. Then $P_{\bullet}^{\prime}=$ $P \bullet{ }_{A} A^{\prime}$ is a cofibrant $A^{\prime}$-algebra, since $A\langle X\rangle \underset{A}{\otimes} A^{\prime} \simeq A^{\prime}\langle X\rangle$ for a set $X$. Since $P_{\bullet}$ is also a complex of projective $A$-modules, it forms a projective $A$-module resolution of $R$. Thus, if $R$ is flat, $P_{\bullet}^{\prime}$ is a cofibrant resolution of $R^{\prime}$.

Using $E_{*} \rightarrow E_{*} / \mathfrak{m}$ and the flatness of $E_{*} F$ over $E_{*}$, we get

COROLlary 19.6. There is an isomorphism

$$
\operatorname{Der}_{E_{*}}^{s}\left(E_{*} F, E_{*+t} / \mathfrak{m}\right) \simeq \operatorname{Der}_{E_{*}}^{s}\left(E_{*} F / \mathfrak{m}, E_{*+t} / \mathfrak{m}\right) .
$$

Using $E_{0} / \mathfrak{m} \rightarrow E_{*} / \mathfrak{m}$ and the fact that $E_{0} / \mathfrak{m}$ is a field, we get

COROLlaRY 19.7. There is an isomorphism

$$
\operatorname{Der}_{E_{*}}^{s}\left(E_{*} F / \mathfrak{m}, E_{*+t} / \mathfrak{m}\right) \simeq \operatorname{Der}_{E_{0} / \mathfrak{m}}^{s}\left(E_{0} F / \mathfrak{m}, E_{t} / \mathfrak{m}\right) .
$$




\section{Reduction to commutative algebras}

20.1. Commutative algebra derivations. We now let $B$ be a commutative $A$-algebra, and let $M$ be a $B$-module. Then $B \oplus M$ can be given the structure of an $A$-algebra augmented over $B$. The sections of this augmentation correspond to (commutative) $A$-algebra derivations of $B$ into $M$. A commutative algebra derivation is the same thing as an associative algebra derivation for which $M$ is viewed as a $B$-bimodule with the same $B$-module structure on both sides.

There are natural isomorphisms

$$
\operatorname{Der}_{A}(B, M) \simeq \operatorname{Hom}_{B^{e}}\left(I_{B / A}, M\right) \simeq \operatorname{Hom}_{B}\left(I_{B / A} \underset{B^{e}}{\otimes} B, M\right)
$$

using the map $B^{e}=B \underset{A}{\otimes} B \rightarrow B$. We call $\Omega_{B / A}=I_{B / A}{\underset{B}{B^{e}}}_{B} B$ the $B$-module of commutative algebra differentials on $B$; there is a universal derivation $d: B \rightarrow$ $\Omega_{B / A}$. Because of the universal property of $\Omega_{B / A}$, we see that $\Omega_{B / A}$ may also be described as the free $B$-module on elements $d x$ for $x \in B$, modulo the relations $d a=0$ for $a \in A$ and $d(x y)=x d y+y d x$. In particular, if $B=A[X]$ is a polynomial algebra generated by a set $X$, then $\Omega_{B / A}$ is a free $B$-module generated by the set of symbols $\{d x \mid x \in X\}$.

The category of simplicial commutative algebras has a model category structure, in which a weak equivalence $B_{\bullet} \rightarrow B_{\bullet}^{\prime}$ is a map which induces an isomorphism on the homology of the associated chain complex, and a cofibration is a retract of a free map. A free map $f: B_{\bullet} \rightarrow B_{\bullet}^{\prime}$ is a map which in each simplicial degree is an inclusion

$$
B_{n} \rightarrow B_{n}^{\prime} \simeq B_{n}\left[X_{n}\right]
$$

where $B[X]$ denotes the polynomial algebra over $B$ generated by elements of $X$, and such that the sets $X_{\bullet}$ are closed under degeneracy operators of $B_{\bullet}^{\prime}$. (See Quillen [11].)

Thus, we can define derived functors of commutative algebra derivations, called André-Quillen cohomology, by

$$
H_{A}^{s}(B, M)=H^{s}\left[\operatorname{Der}_{A}\left(Q_{\bullet}, M\right)\right]
$$

where $Q_{\bullet} \rightarrow B$ is a cofibrant resolution of $B$ as a commutative algebra. (This is Quillen's construction of André-Quillen cohomology; see Quillen [12].)

If $Q_{\bullet} \rightarrow B$ is a cofibrant commutative algebra resolution of $B$, write $L_{B / A}=$ $\Omega_{Q \bullet / A} \otimes B$. Then

$$
H_{A}^{s}(B, M) \simeq H^{s}\left[\operatorname{Hom}_{B}\left(L_{B / A}, M\right)\right]
$$

20.2. Comparing associative and commutative derivations. Let's fix a commutative $A$-algebra $B$ and a $B$-module $M$. Then let

$$
\Lambda_{B / A} \simeq D_{B / A} \underset{B^{e}}{\otimes} B
$$

Thus, $\Lambda_{-/ A}$ is a construction which produces a simplicial $B$-module from the commutative $A$-algebra $B$. It has the property that

$$
\operatorname{Der}_{A}^{s}(B, M) \simeq H^{s}\left[\operatorname{Hom}_{B}\left(\Lambda_{B / A}, M\right)\right]
$$

On the other hand, we have the gadget $L_{B / A}$ which is another simplicial $B$-module. We want to show that if $L_{B / A}$ is acyclic (i.e., $H_{s} L_{B / A} \equiv 0$ for all $s$ ), then $\Lambda_{B / A}$ is acyclic. In fact 
Proposition 20.3. If $B$ is a commutative ring which is flat over $A$, then there is a first quadrant spectral sequence

$$
H_{s}\left(\Lambda_{B}^{t+1}\left(L_{B / A}\right)\right) \Longrightarrow H_{s+t} \Lambda_{B / A}
$$

(Here $\Lambda_{B}^{d}$ denotes the $d$ th exterior power functor; $\Lambda_{B}^{t+1} L_{B / A}$ is the simplicial $B$-module obtained by applying the $(t+1)$ st exterior power functor degree-wise to $\left.L_{B / A}\right)$

Corollary 20.4. Let $A=E_{0} / \mathfrak{m}=k_{1}$ and $B=E_{0} F / \mathfrak{m}$. If $L_{B / A}$ is acyclic, then $\Lambda_{B / A}$ is acyclic, whence

$$
\operatorname{Der}_{E_{0} / \mathfrak{m}}^{s}\left(E_{0} F / \mathfrak{m}, E_{t}\right)=0 .
$$

Proof. Since $L_{B / A}$ is an acyclic simplicial module which is degree-wise projective, it has a simplicial contracting homotopy. Hence so does $\Lambda_{B}^{t+1} L_{B / A}$, whence the result follows by Proposition 20.3.

20.5. Construction of the spectral sequence. First, let $Q_{\bullet} \rightarrow B$ be a vertical cofibrant resolution of $B$ as a commutative algebra; without loss of generality we can assume that each $Q_{p}$ is a polynomial algebra. Now, let $P_{p, \bullet} \rightarrow Q_{p}$ be a horizontal functorial cofibrant resolution of $Q_{p}$ as an associative algebra. Hence, we get a double complex $P_{\bullet}, \bullet$ of associative algebras; the diagonal of this double complex is a cofibrant resolution of $B$ as an associative algebra.

We apply the functor $P \mapsto I_{P / A} \underset{P^{e}}{\otimes} B$ to each spot in the double complex. The total homology of the resulting complex of $B$ modules is precisely $H_{*}\left(\Lambda_{B / A}\right)$. Taking horizontal homology in the complex we get

$$
E_{1}^{p, q}=H_{q}\left(I_{P_{p}, \bullet} \underset{P_{p}^{e}, \bullet}{\otimes} B\right) \simeq H_{q}\left(\Lambda_{Q_{p} / A} \underset{Q_{p}}{\otimes} B\right) .
$$

LEMma 20.6. Given a flat commutative A-algebra $Q$ there is a natural isomorphism

and a natural map

$$
\operatorname{Tor}_{q+1}^{Q^{e}}(Q, Q) \stackrel{\sim}{\longrightarrow} H_{q}\left(\Lambda_{Q / A}\right),
$$

$$
i: \Omega_{Q / A} \stackrel{\sim}{\longrightarrow} H_{0} \Lambda_{Q / A} .
$$

Lemma 20.6 is proved below.

Note that $\operatorname{Tor}_{*}^{Q^{e}}(Q, Q)$ has the structure of a graded commutative algebra. This algebra structure arises from the fact that there are algebra maps $Q \rightarrow Q^{e}=$ $Q \underset{A}{\otimes} Q \rightarrow Q$. Furthermore, if $Q$ is a polynomial algebra, then $\operatorname{Tor}_{*}^{Q^{e}}(Q, Q)$ is an exterior algebra generated by Tor $_{1}$. Thus, in the case that $Q$ is a polynomial algebra over $A$, (whence $Q^{e}$ is a polynomial algebra over $Q$,) the natural map $i$ extends in a natural way to an isomorphism of algebras

$$
\Lambda_{B}^{*+1}\left(\Omega_{Q / A}\right) \stackrel{\sim}{\longrightarrow} \operatorname{Tor}_{*+1}^{Q^{e}}(Q, Q) \stackrel{\sim}{\longrightarrow} H_{*}\left(\Lambda_{Q / A}\right) .
$$

In particular,

whence

$$
H_{q}\left(\Lambda_{Q_{p} / A}\right) \simeq \Lambda_{Q_{p}}^{q+1}\left(\Omega_{Q_{p} / A}\right)
$$

$$
E_{1}^{p, q} \simeq \Lambda_{Q_{p}}^{q+1}\left(\Omega_{Q_{p} / A}\right) \underset{Q_{p}}{\otimes} B \simeq \Lambda_{B}^{q+1}\left(\Omega_{Q_{p} / A} \underset{Q_{p}}{\otimes} B\right)
$$


(This last follows because $\Lambda_{Q_{p}}^{*}\left(\Omega_{Q_{p} / A}\right)$ is a projective $Q_{p}$-module.) The right-hand side of this equation is the $p$ th degree of $\Lambda_{B}^{q+1} L_{B / A}$, and thus Proposition 20.3 holds.

Proof of Lemma 20.6. Recall that $\Lambda_{Q / A}=I_{P_{\bullet} / A} \underset{P_{\bullet}^{e}}{\otimes} Q$ where $P_{\bullet} \rightarrow Q$ is a cofibrant resolution of $Q$ by associative algebras, and that there are exact sequences

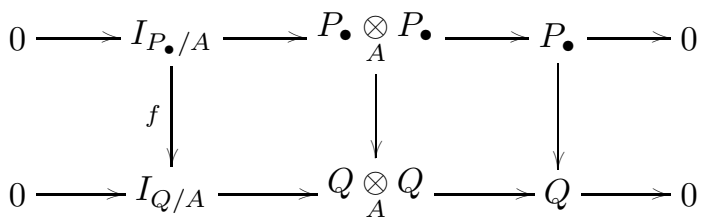

The right-hand vertical arrow induces an isomorphism on homology by construction. Since $Q$ is flat over $A$ the middle vertical arrow also induces an equivalence on homology. Hence by the 5-lemma the map $f$ is an equivalence.

This map $f$ factors into a sequence of maps of simplicial modules

$$
I_{P_{\bullet} / A} \simeq I_{P_{\bullet} / A} \underset{P_{\bullet}^{e}}{\otimes} P_{\bullet}^{e} \rightarrow I_{P_{\bullet} / A} \underset{P_{\bullet}^{e}}{\otimes} Q^{e} \rightarrow I_{Q / A}
$$

The left-hand arrow in this sequence is an equivalence by a straightforward spectral sequence argument (since $I_{P_{\bullet} / A}$ is a projective $P_{\bullet}^{e}$-module). Since the composite arrow is an equivalence, it follows that $I_{P_{\bullet} / A} \underset{P_{\bullet}^{e}}{\otimes} Q^{e}$ is a projective $Q$-bimodule resolution of $I_{Q / A}$.

Hence the complex $I_{P_{\bullet} / A} \underset{P_{\bullet}^{e}}{\otimes} Q^{e} \underset{Q^{e}}{\otimes} Q \simeq I_{P_{\bullet} / A} \underset{P_{\bullet}}{\otimes} Q$ (which is precisely $\Lambda_{Q / A}$ ) computes $\operatorname{Tor}_{*}^{Q^{e}}\left(I_{Q / A}, Q\right)$. Using the long exact sequence of Tor applied to the sequence $0 \rightarrow I_{Q / A} \rightarrow Q^{e} \rightarrow Q \rightarrow 0$, and the fact that $\operatorname{Tor}_{*}^{Q^{e}}\left(Q^{e}, Q\right)$ vanishes for $*>0$, we see that

as desired.

$$
\operatorname{Tor}_{q+1}^{Q^{e}}(Q, Q) \stackrel{\sim}{\longrightarrow} H_{q}\left(\Lambda_{Q / A}\right)
$$

\section{End of the calculation}

In this section we show that the André-Quillen cohomology groups, denoted $H_{E_{0} / \mathfrak{m}}^{s}\left(E_{0} F / \mathfrak{m}, E_{t}\right)$, vanish, which by Corollary 20.4, Corollary 19.4, Corollary 19.6, and Corollary 19.3 proves part (2b) of Theorem 14.5.

21.1. Differentials and the Frobenius map. Suppose that $A$ is an $\mathbb{F}_{p^{-}}$ algebra, and $B$ an $A$-algebra. Then the map $\sigma_{B}: B \rightarrow B$ defined by $\sigma_{B}(x)=x^{p}$ is a homomorphism. For an algebra map $A \rightarrow B$ we get a commutative diagram

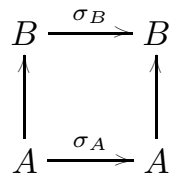


Of course, $\sigma_{B}$ is not in general a map of $A$-algebras. Let $\sigma^{*} B$ denote the pushout of commutative rings in

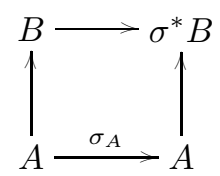

Then there is an $A$-algebra map $\phi: \sigma^{*} B \rightarrow B$ (the Frobenius map). If $B=A[X]$ is a polynomial algebra over $A$ generated by the set $X$, then $\sigma^{*} B \simeq A[X]$, and $\phi$ sends $x \in X$ to $x^{p}$.

Proposition 21.2. For any A-algebra $B$ as above, the map

$$
L_{\sigma^{*} B / A} \rightarrow L_{B / A}
$$

induced by $\phi: \sigma^{*} B \rightarrow B$ is the 0 map.

Proof. Let $Q_{\bullet} \rightarrow B$ be a cofibrant commutative $A$-algebra resolution of $B$. We may suppose that $Q_{n}=A\left[X_{n}\right]$ for some set $X_{n}$. Then $\sigma^{*} Q \bullet \rightarrow \sigma^{*} B$ is a commutative $A$-algebra resolution of $\sigma^{*} B$. If we apply the differentials functor $\Omega_{-/ A}$ we get induced maps which send

$$
d x \mapsto d \phi(x)=d\left(x^{p}\right)=p x^{p-1} d x=0
$$

for each $x \in X_{n} \subset \sigma^{*} Q_{n}$. Thus, on the level of complexes, we get the zero map $L_{\sigma^{*} B / A} \rightarrow L_{B / A}$.

Corollary 21.3. If $B$ is an A-algebra and if the maps $\sigma_{A}$ and $\sigma_{B}$ are isomorphisms, then $H_{*}\left(L_{B / A}\right) \equiv 0$.

Proof. If $\sigma_{A}$ and $\sigma_{B}$ are isomorphisms, then $\phi: \sigma^{*} B \rightarrow B$ is an isomorphism of $A$-algebras, whence $L \phi$ is an equivalence. But $L \phi \sim 0$ by Proposition 21.2.

21.4. The Frobenius map on $E_{0} F / \mathfrak{m}$. Recall from Remark 17.4 that if $E=F=E_{n}$, then one can compute that

$$
B=E_{0} F / \mathfrak{m} \simeq\left(\mathbb{F}_{p^{n}} \underset{\mathbb{F}_{p}}{\otimes} \mathbb{F}_{p^{n}}\right)\left[e_{0}, e_{1}, e_{2}, \ldots\right] /\left(e_{0}^{p^{n}-1}-1, e_{1}^{p^{n}}-e_{1}, e_{2}^{p^{n}}-e_{2}, \ldots\right) .
$$

Clearly the Frobenius maps on $A=\mathbb{F}_{p^{n}}$ and on $B$ are isomorphisms, so in this case we are done.

More generally, let $E=E_{k_{1}, \Gamma_{1}}$ and $F=E_{k_{2}, \Gamma_{2}}$, and let $A=E_{0} / \mathfrak{m}=k_{1}$, $B=E_{0} F / \mathfrak{m}$ and let $Q$ be a commutative ring of characteristic $p$. Then given a map of rings $f: B \rightarrow Q$ there is a commutative diagram

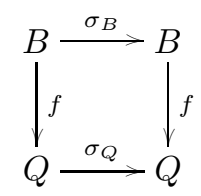

In other words, there are two maps

$$
\left(\sigma_{B}\right)_{*},\left(\sigma_{Q}\right)^{*}: \operatorname{Hom}_{\text {Ring }}(B, Q) \rightrightarrows \operatorname{Hom}_{\operatorname{Ring}}(B, Q) \text {; }
$$

the top map takes $f$ to $\sigma_{Q} f$, the bottom map to $f \sigma_{B}$, and $\left(\sigma_{B}\right)_{*}=\left(\sigma_{Q}\right)^{*}$. Thus to show that $\sigma_{B}$ is an isomorphism, it suffices to show that $\left(\sigma_{Q}\right)^{*}$ is an isomorphism for all $Q$. 
Recall (from Remark 17.2) our homotopy pull-back square

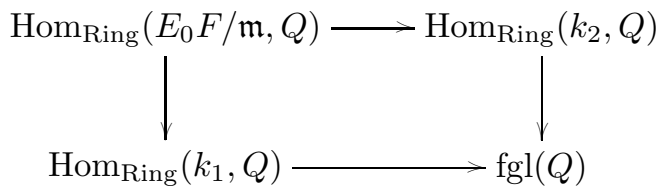

which is functorial in $Q$. The automorphism $\sigma_{Q}$ induces a map between such squares. Since $k_{1}$ and $k_{2}$ are perfect fields, the induced $p$ th-power maps $\left(\sigma_{Q}\right)^{*}$ on $\operatorname{Hom}_{\text {Ring }}\left(k_{1}, Q\right)$ and $\operatorname{Hom}_{\text {Ring }}\left(k_{2}, Q\right)$ are isomorphisms.

Let $\operatorname{fgl}_{n}(Q) \subset \operatorname{fgl}(Q)$ denote the full subgroupoid of formal group laws of height $n$. (Although $Q$ is only a ring of characteristic $p$, and not in general a field, we define height as before: a formal group law $F$ has height $n$ if $[p]_{F}(x)=a x^{p^{n}}+\cdots$ where $a \in Q$ is an invertible element. Under this definition it is possible for a formal group law over $Q$ to not have a well-defined height. Note however that if $f: F \rightarrow G$ is an isomorphism of formal group laws over $Q$, and if $F$ is of height $n$, then $G$ is also of height $n$, since $[p]_{G}=f \circ[p]_{F} \circ f^{-1}$.)

Proposition 21.5. There is a natural isomorphism of the functor

$$
\left(\sigma_{Q}^{n}\right)^{*}: \operatorname{fgl}_{n}(Q) \rightarrow \operatorname{fgl}_{n}(Q)
$$

with the identity functor, whence $\sigma_{Q}^{*}$ is an equivalence of categories.

Corollary 21.6. The Frobenius map on $E_{0} F / \mathfrak{m}$ is an isomorphism.

Recall that there is a homomorphism $\alpha_{F}: F \rightarrow \sigma^{*} F$ of formal group laws given by $\alpha_{F}(x)=x^{p}$.

Lemma 21.7. Given a map $f: F \rightarrow G$ of formal group laws over a ring of characteristic $p$, such that $f^{\prime}(0)=0$, there exists a unique map $g: \sigma^{*} F \rightarrow G$ of formal group laws such that $g \circ \alpha_{F}=f$.

Proof. Because $f(F(x, y))=G(f x, f y)$, applying $\frac{\partial}{\partial y}$ and setting $y=0$ gives $f^{\prime}(x) F_{2}(x, 0)=G_{2}(f x, 0) f^{\prime}(0)$, whence $f^{\prime}(x)=0$. Thus $f(x)=g\left(x^{p}\right)$ for a (unique) power series $g$. Since

$$
g\left(\sigma^{*} F\left(x^{p}, y^{p}\right)\right)=g\left(F(x, y)^{p}\right)=f(F(x, y))=G(f x, f y)=G\left(g\left(x^{p}\right), G\left(y^{p}\right)\right)
$$

we see that $g(x \underset{F}{+} y)=g(x) \underset{G}{+} g(y)$.

Proof of Proposition 21.5. If $F$ is a formal group law of height $n$, then the $p$-series $[p]_{F}(x): F \rightarrow F$ has the form $[p]_{F}(x)=a x^{p^{n}}+\cdots$, with $a$ invertible. Thus by Lemma 21.7 there exists a unique isomorphism $\beta_{F}:\left(\sigma^{n}\right)^{*} F \rightarrow F$ such that $\beta_{F} \circ \alpha_{F}^{n}=[p]_{F}$.

If $h: F \rightarrow G$ is a map between height $n$ formal group laws, we see that

$$
h\left(\beta_{F}\left(x^{p^{n}}\right)\right)=h\left([p]_{F}(x)\right)=[p]_{G}(h(x))=\beta_{G}\left(h(x)^{p^{n}}\right)=\beta_{G}\left(h\left(x^{p^{n}}\right)\right),
$$

whence $h \circ \beta_{F}=\beta_{G} \circ h$, whence $\beta:\left(\sigma^{n}\right)^{*} \rightarrow$ id is an natural isomorphism of functors. Thus $\left(\sigma^{n}\right)^{*}$ is an equivalence of categories. 


\section{Part 4. Construction of the $\mathcal{A}_{\infty}$-ring structures}

In the following sections we show that the homology theories introduced in Section 6 admit $\mathcal{A}_{\infty}$-ring structures, by showing that certain obstructions to the existence of such structures vanish. This technique originated in work of Robinson [15] on the existence of $\mathcal{A}_{\infty}$-structures for Morava $K$-theories (see also Remark 23.8).

\section{Producing an algebra structure}

We still need to show that for each formal group law $(k, \Gamma)$ the ring spectrum $E=E_{k, \Gamma}$ admits an $\mathcal{A}_{\infty}$-structure. That is, for an appropriate $\mathcal{A}_{\infty}$-operad $C$, we want a map of operads making the diagram

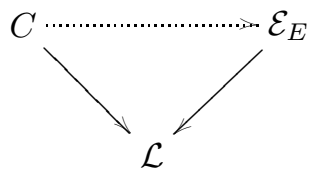

commute. Recall from Section 12.1 that $\mathcal{E}_{E}$ is an operad with $\mathcal{E}_{E}[n]=\mathcal{S}\left(E^{(n)}, E\right)$.

We will choose our operad $C$ so that it is a cofibrant $\mathcal{A}_{\infty}$ operad.

22.1. Free operads. If $A$ is a $\Sigma$-object in some symmetric monoidal category, the free operad $\mathcal{F} A$ on $A$ is an operad equipped with a map of $\Sigma$-objects $A \rightarrow \mathcal{F} A$ inducing an isomorphism

$$
\operatorname{Hom}_{\text {oper }}(\mathcal{F} A, C) \simeq \operatorname{Hom}_{\Sigma}(A, C),
$$

for each operad $C$. There is a free operad functor which is left adjoint to the forgetful functor from operads to $\Sigma$-objects.

Since operads are monoids in the category of $\Sigma$-objects, the free operad should really be a kind of "free monoid". This is true, but the formula for such a free monoid is somewhat strange. Morally speaking,

$$
\mathcal{F} A=I \amalg A \circ(I \amalg A \circ(I \amalg A \circ(\cdots))) .
$$

More precisely, $\mathcal{F} A$ can be written as a countable colimit of expressions of the above form which terminate at some finite depth.

The reason for this strange formula is that the monoidal structure $\circ$ on $\Sigma$ objects is not "bi-distributive"; rather, it distributes over coproducts on the right, but not on the left. I.e., in general $(A \amalg B) \circ C \simeq(A \circ C) \amalg(B \circ C)$, but $A \circ(B \amalg C) \not$ $(A \circ B) \amalg(A \circ C)$.

22.2. Model category for operads. We give a model category structure for the category $s$ oper of simplicial operads.

Say a $\Sigma$-object $A$ is $\Sigma$-free if each $A[n]$ is free as a $\Sigma_{n}$-simplicial set. We define a closed model category for simplicial operads by letting $f: A \rightarrow B$ be

1. a weak equivalence if each map $f[n]: A[n] \rightarrow B[n]$ is a weak equivalence on the underlying simplicial set, and

2. a cofibration if it is a retract of a free map. A free map is a map $f$ of the form

$$
f_{n}: A_{n} \rightarrow B_{n} \simeq A_{n} \underset{\text { oper }}{\amalg} \mathcal{F} X_{n}
$$

where $X_{n}$ is a discrete, $\Sigma$-free $\Sigma$-object, and such that the subobjects $X_{n} \subset$ $B_{n}$ are closed under degeneracy operators. 
That this indeed gives a model category structure follows from general results of Quillen [11].

To produce our desired operad $C$, first let $A \rightarrow \mathcal{L}$ be an arbitrary $\mathcal{A}_{\infty}$-operad over the linear isometries operad. Let $\operatorname{Sing} A$ be the simplicial operad obtained by taking singular simplices, and let $\tilde{C} \rightarrow \operatorname{Sing} A$ be a cofibrant resolution of $\operatorname{Sing} A$; we can choose $\tilde{C}$ so that $\tilde{C}_{n}=\mathcal{F} K_{n}$ for some $\Sigma$-free $\Sigma$-object $K_{n}$. Then by adjunction we get a map $C=|\tilde{C}| \rightarrow A$ which is a weak equivalence of topological operads.

22.3. The spectral sequence. We want to show that $\operatorname{map}_{(\operatorname{oper} \downarrow \mathcal{L})}\left(C, \mathcal{E}_{E}\right)$ is non-empty. The simplicial object $\tilde{C}_{n}=\mathcal{F} K_{n}$ leads to a cosimplicial space $Y^{\bullet}$, with

$$
Y^{n}=\operatorname{map}_{(\operatorname{oper} \downarrow \mathcal{L})}\left(\mathcal{F} K_{n}, \mathcal{E}_{E}\right),
$$

with $\operatorname{Tot} Y^{\bullet}=\operatorname{map}_{(\operatorname{oper} \downarrow \mathcal{L})}\left(|\tilde{C}|, \mathcal{E}_{E}\right)$. Thus, if we choose an element $\sigma \in \pi_{0} Y^{0} \rightrightarrows$ $\pi_{0} Y^{1}$, there is an obstruction theory / spectral sequence of the cosimplicial space (see Section 14) with

$$
\left.E_{2}^{s, t}=\pi^{s} \pi_{t}\left(\operatorname{map}_{(\operatorname{oper} \downarrow \mathcal{L})}\left(\mathcal{F} K_{n}, \mathcal{E}_{E}\right), \sigma\right)\right) .
$$

22.4. Identification of $E_{2}^{0,0}$. We need to compute the equalizer of the pair of arrows

$$
\pi_{0} \operatorname{map}_{(\operatorname{oper} \downarrow \mathcal{L})}\left(\mathcal{F} K_{0}, \mathcal{E}_{E}\right) \rightrightarrows \pi_{0} \operatorname{map}_{(\operatorname{oper} \downarrow \mathcal{L})}\left(\mathcal{F} K_{1}, \mathcal{E}_{E}\right)
$$

Note that for any operad $B$ over $\mathcal{L}$ there is a natural map

$$
\pi_{0} \operatorname{map}_{(\operatorname{oper} \downarrow \mathcal{L})}\left(B, \mathcal{E}_{E}\right) \rightarrow \operatorname{Hom}_{\text {oper }}\left(\pi_{0} B, \pi_{0} \mathcal{E}_{E}\right)
$$

which sends an operad map $f: B \rightarrow \mathcal{E}_{E}$ over $\mathcal{L}$ to the map $\pi_{0} f: \pi_{0} B \rightarrow \mathcal{E}_{E}$, where $\pi_{0} B$ denotes the discrete operad with $\left(\pi_{0} B\right)[n]=\pi_{0}(B[n])$.

Since $\mathcal{E}_{E}[n] \simeq \mathcal{S}\left(E^{(n)}, E\right)$, we see that $\pi_{0} \mathcal{E}_{E}[n] \simeq\left[E^{(n)}, E\right]$. In fact, by Proposition 15.5 ,

$$
\pi_{0} \mathcal{E}_{E}[n] \simeq \operatorname{Hom}_{E_{*}}\left(\left(E_{*} E\right)^{\otimes n}, E_{*}\right) .
$$

Now suppose $B=\mathcal{F} K$ for some discrete $\Sigma$-object $K$ equipped with a map $K \rightarrow \mathcal{L}$. Such a $K$ is necessarily $\Sigma$-free, since $\mathcal{L}$ is. There is a commutative diagram

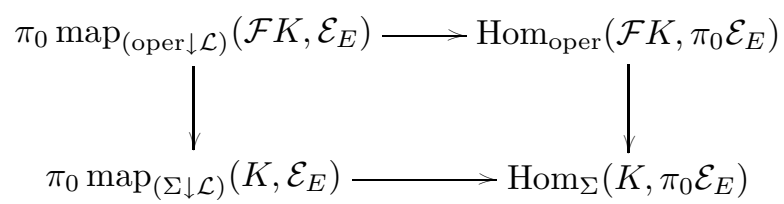

The vertical arrows are isomorphisms by the characteristic property of a free operad. The lower horizontal arrow is also an isomorphism, since $K$ is discrete and $\Sigma$-free. Thus the upper horizontal arrow is an isomorphism. Thus we see that $E_{2}^{0,0}$ is isomorphic to the equalizer of the pair of arrows

$$
E_{2}^{0,0} \rightarrow \operatorname{Hom}_{\text {oper }}\left(\mathcal{F} K_{0}, \pi_{0} \mathcal{E}_{E}\right) \rightrightarrows \operatorname{Hom}_{\text {oper }}\left(\mathcal{F} K_{1}, \pi_{0} \mathcal{E}_{E}\right)
$$

Since $\pi_{0} C$, the coequalizer of $\mathcal{F} K_{1} \rightrightarrows \mathcal{F} K_{0}$, is the associative monoid operad, it follows that $E_{2}^{0,0} \simeq \operatorname{Hom}_{\text {oper }}\left(\pi_{0} C, \pi_{0} \mathcal{E}_{E}\right)$. This is precisely the set of ring spectrum structures on $E$; that is, an associative ring spectrum structure on $E$ (in the "classical" sense, rather than in the $\mathcal{A}_{\infty}$ sense) is precisely equivalent to a map from the associative operad to $\pi_{0} \mathcal{E}_{E}$. 
22.5. Identification of $E_{2}$-term. Let us now choose the element $\sigma \in E_{2}^{0,0}$ corresponding to the "usual" ring spectrum structure on $E$. We obtain as before an obstruction theory / spectral sequence with

$$
E_{1}^{s, t} \simeq \pi_{t}\left(\operatorname{map}_{(\operatorname{oper} \downarrow \mathcal{L})}\left(\mathcal{F} K_{s}, \mathcal{E}_{E}\right), \sigma\right)
$$

and "converging" to $\pi_{t-s}\left(\operatorname{map}_{(\operatorname{oper} \downarrow \mathcal{L})}\left(C, \mathcal{E}_{E}\right), \sigma\right)$.

THEOREM 22.6. The $E_{2}$-term of this spectral sequence is given by

$$
E_{2}^{s, t}= \begin{cases}0 & \text { if } s>0, \\ {\left[\Sigma^{t} E, E\right]} & \text { if } s=0, t>0 .\end{cases}
$$

Proof. This follows from the discussion of Section 23.1, Theorem 23.5, Remark 23.6, and Proposition 23.7.

In particular, the obstruction groups $E_{2}^{s, s-1}$ vanish identically, and so

COROLLARY 22.7. The spectrum $E$ admits an $A_{\infty}$-ring structure whose underlying ring structure is equivalent to the usual one.

\section{Computation of algebra-structure obstruction groups}

If $B$ is an operad and $K$ is an unpointed space, let $\operatorname{map}(K, B)$ denote the operad with $\operatorname{map}(K, B)[n]=\operatorname{map}(K, B[n])$, and with operad structure given by "point-wise composition". Now if given a map $B \rightarrow \mathcal{L}$ of operads, define an operad $B^{K}$ over the linear isometries operad via the pullback square

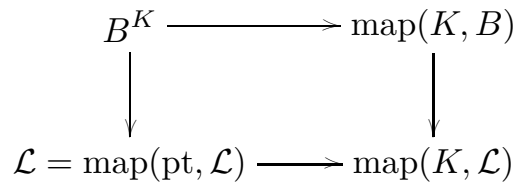

To prove Theorem 22.6, we must first compute the $E_{1}$-term. Suppose that $B \rightarrow \mathcal{L}$ is a map of operads, and $\sigma: B \rightarrow \mathcal{E}_{E}$ is some fixed map of operads over $\mathcal{L}$. Then the set

$$
\pi_{t}\left(\operatorname{map}_{(\operatorname{oper} \downarrow \mathcal{L})}\left(B, \mathcal{E}_{E}\right), \sigma\right)
$$

is the same as the set of homotopy classes of maps $S^{t} \rightarrow \operatorname{map}_{(\operatorname{oper} \downarrow \mathcal{L})}\left(B, \mathcal{E}_{E}\right)$ which send the base-point of the sphere to $\sigma$, and where homotopies are base-point preserving.

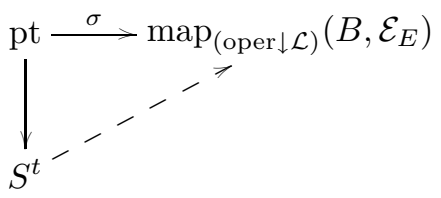

By adjunction, these are the same as homotopy classes of lifts in the diagram

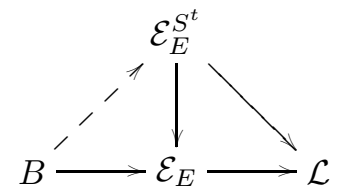

That is, there is a natural isomorphism

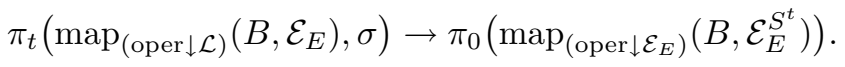


There is a natural map

$$
\pi_{0} \operatorname{map}_{(\operatorname{oper} \downarrow \mathcal{L})}\left(B, \mathcal{E}_{E}^{S^{t}}\right) \rightarrow \operatorname{Hom}_{\text {oper }}\left(\pi_{0} B, \pi_{0} \mathcal{E}_{E}\right)
$$

If $B=\mathcal{F} K$ and $K$ is discrete and $\Sigma$-free there is a square

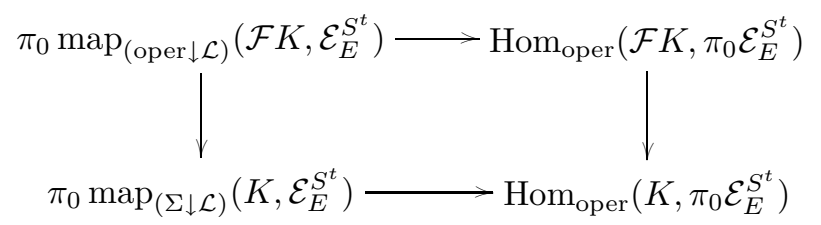

The vertical arrows are isomorphisms by the universal property of free operads. The lower horizontal arrow is an isomorphism since $K$ is discrete and $\Sigma$-free. Thus the top horizontal arrow is an isomorphism. This isomorphism, and the fact that the map $\mathcal{E}_{E}^{S^{t}} \rightarrow \mathcal{E}_{E}$ is a split fibration (using the base-point of $S^{t}$ ), implies that the map is an isomorphism.

$$
\pi_{0}\left(\operatorname{map}_{\left(\text {oper } \downarrow \mathcal{E}_{E}\right)}\left(\mathcal{F} K, \mathcal{E}_{E}^{S^{t}}\right)\right) \rightarrow \operatorname{map}_{\left(\text {oper } \downarrow \pi_{0} \mathcal{E}_{E}\right)}\left(\mathcal{F} K, \pi_{0} \mathcal{E}_{E}^{S^{t}}\right)
$$

Since $\mathcal{E}_{E}^{S^{t}}[n]$ is weakly equivalent to $\operatorname{map}\left(S^{t}, \mathcal{E}_{E}[n]\right)$, it follows that

$$
\pi_{0} \mathcal{E}_{E}^{S^{t}}[n]=\operatorname{Hom}_{E_{*}}\left(\left(E_{*} E\right)^{\otimes n}, E_{*}[\epsilon] / \epsilon^{2}\right),
$$

where $|\epsilon|=-t$; furthermore, the projection map $\pi_{0} \mathcal{E}_{E}^{S^{t}} \rightarrow \pi_{0} \mathcal{E}_{E}$ sends $\epsilon$ to 0 .

Since the pinch map $S^{t} \rightarrow S^{t} \vee S^{t}$ makes the sphere into a cogroup up to homotopy, the induced map of operads

$$
\pi_{0} \mathcal{E}_{E}^{S^{t}} \underset{\pi_{0} \mathcal{E}_{E}}{\times} \pi_{0} \mathcal{E}_{E}^{S^{t}} \simeq \pi_{0} \mathcal{E}_{E}^{S^{t} \vee S^{t}} \rightarrow \pi_{0} \mathcal{E}_{E}^{S^{t}}
$$

makes $\pi_{0} \mathcal{E}_{E}^{S^{t}}$ into an abelian group object in the category of operads over $\pi_{0} \mathcal{E}_{E}$.

23.1. Quillen cohomology of an operad. Suppose $A \rightarrow B$ is a map of discrete operads. Let $C$. be a cofibrant resolution of a discrete operad $A$. Let $N \rightarrow B$ be an abelian group operad over an operad $B$. Then we define the Quillen cohomology of an operad by

$$
H^{s}(A, N)=H^{s}\left[\operatorname{Hom}_{(\operatorname{oper} \downarrow B)}\left(C_{\bullet}, N\right)\right] .
$$

In our case we take $A$ to be the associative operad, $B=\pi_{0} \mathcal{E}_{E}$, and we take $N=\pi_{0} \mathcal{E}_{E}^{S^{t}}$ for $t>0$. Thus we have that

$$
E_{2}^{s, t}=H^{s}\left(A, \pi_{0} \mathcal{E}_{E}^{S^{t}}\right)
$$

23.2. Bimodules. Let $A$ be an operad. An $A$-bimodule is a $\Sigma$-object $M$ equipped with maps $\ell: A \circ M \rightarrow M$ and $r: M \circ A \rightarrow M$ such that the diagrams
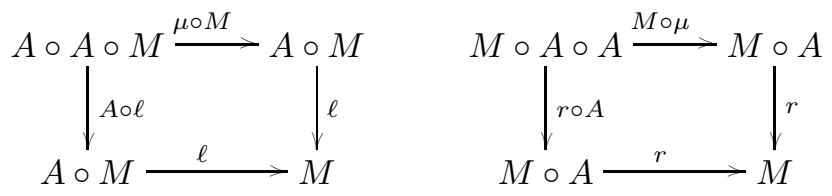
and

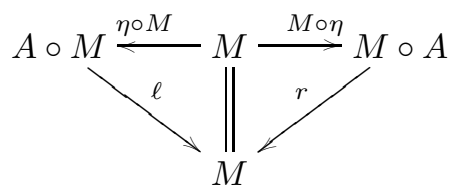

commute.

There are several examples of bimodules.

1. $A$ is itself an $A$-bimodule.

2. If $K$ is a $\Sigma$-object, then $A \circ K \circ A$ is an $A$-bimodule, called the free $A$-bimodule on $K$

3. If $X$ and $Y$ are objects, define a $\Sigma$-object $\mathfrak{h o m}(X, Y)$ by

$$
\mathfrak{h o m}(X, Y)[n]=\operatorname{Hom}\left(X^{\otimes n}, Y\right) .
$$

If $X=Y$, then $\mathfrak{h o m}(X, X)$ is the endomorphism operad of $X$. If $X$ and $Y$ are $A$-algebras, then $\mathfrak{h o m}(X, Y)$ is an $A$-bimodule.

An $A$-bimodule $M$ induces a functor from $A$-algebras to $A$-algebras as follows. Define $M \underset{A}{\circ}(X)$ by the coequalizer

$$
M \stackrel{\circ}{\circ}(X)=\operatorname{Cok}((M \circ A)(X) \rightrightarrows M(X)) .
$$

Note that if $M=A$, then $A \circ_{A}(X) \simeq X$; i.e., the bimodule $A$ induces the identity functor on $A$-algebras. Likewise, if $M=A^{\text {od }}$, then $A_{A}^{\circ d}{ }_{A}^{\circ}(X) \simeq A^{d-1}(X)$. There is an adjunction

$$
\operatorname{Hom}_{A-\operatorname{bimod}}(M, \mathfrak{h o m}(X, Y)) \simeq \operatorname{Hom}_{A \text {-alg }}\left(M \circ \circ_{A}(X), Y\right) .
$$

23.4. Bar complex. If $M$ and $N$ are $A$-bimodules, let $\mathcal{B}(M, A, N)$ denote the simplicial object

$$
M \circ N \leftleftarrows M \circ A \circ N \leftleftarrows M \circ A \circ A \circ N \sum \ldots
$$

In particular, $\mathcal{B}(A, A, A)$ is a resolution of $A$ by free $A$-bimodules.

If $N \rightarrow B$ is a coefficient system (i.e., an abelian group object over $B$ ), then we define the Hochschild cohomology of $A$ by

$$
H H^{s}(A, N)=H^{s}\left[\operatorname{Hom}_{(A-\operatorname{bimod} \downarrow B)}(\mathcal{B}(A, A, A), N)\right] .
$$

The fact that relates the Quillen cohomology and Hochschild cohomology of an operad is the following.

Theorem 23.5. [14] If $A$ is a discrete, $\Sigma$-free operad, and $N$ is a coefficient system for $A$, then there is an exact sequence

$$
0 \rightarrow H H^{0}(A, N) \rightarrow H H^{0}(I, N) \rightarrow H^{0}(A, N) \rightarrow H H^{1}(A, N) \rightarrow 0
$$

and isomorphisms

for $s>0$.

$$
H^{s}(A, N) \simeq H H^{s+1}(A, N)
$$


REMARK 23.6. Recall that $I$ denotes the "trivial operad". Since $I \circ I \simeq I$, the bar complex $\mathcal{B}(I, I, I)$ is constant, so the Hochschild cohomology of $I$ is concentrated in degree 0 . In the case of interest to us, in which the coefficient system $N$ is $\pi_{0} \mathcal{E}_{E}^{S^{t}} \rightarrow \pi_{0} \mathcal{E}_{E}$ pulled back along $I \rightarrow \pi_{0} \mathcal{E}_{E}$, it is trivial to show that

$$
\begin{aligned}
H H^{0}(I, N) & \simeq \operatorname{Hom}_{\left(I-\operatorname{bimod} \downarrow \pi_{0} \mathcal{E}_{E}\right)}\left(I, \pi_{0} \mathcal{E}_{E}^{S^{t}}\right) \\
& \simeq \operatorname{Hom}_{E_{*}}\left(E_{*} E, E_{*+t}\right) \\
& \simeq\left[\Sigma^{t} E, E\right],
\end{aligned}
$$

and that

$$
H H^{s}(I, N)=0 \text { for } s>0 .
$$

Proposition 23.7. If $A$ is the associative operad, then $H H^{s}\left(A, \pi_{0} \mathcal{E}_{E}^{S^{t}}\right) \simeq 0$ for all $s \geq 0$ and $t>0$.

Proof. These cohomology groups are computed using the co-chain complex

$$
C^{n}=\operatorname{Hom}_{\left(A-\operatorname{bimod} \downarrow \pi_{0} \mathcal{E}_{E}\right)}\left(A^{\circ n+2}, \pi_{0} \mathcal{E}_{E}^{S^{t}}\right) .
$$

Note that

$$
\begin{aligned}
\operatorname{Hom}_{A-\operatorname{bimod}}\left(A^{\circ n+2}, \pi_{0} \mathcal{E}_{E}^{S^{t}}\right) & \simeq \operatorname{Hom}_{A-\operatorname{bimod}}\left(A^{\circ n+2}, \mathfrak{h o m}_{E_{*}}\left(E_{*} E, E_{*}[\epsilon] / \epsilon^{2}\right)\right) \\
& \simeq \operatorname{Hom}_{A-\operatorname{alg}}\left(A^{\circ n+2}{ }_{A}^{\circ}\left(E_{*} E\right), E_{*}[\epsilon] / \epsilon^{2}\right) \\
& \left.\simeq \operatorname{Hom}_{E_{*}-\operatorname{alg}}\left(T^{n+1}\left(E_{*} E\right), E_{*}[\epsilon] / \epsilon^{2}\right)\right),
\end{aligned}
$$

using the adjunction of (23.3). Likewise,

$$
\operatorname{Hom}_{A-\operatorname{bimod}}\left(A^{\circ n+2}, \pi_{0} \mathcal{E}_{E}\right) \simeq \operatorname{Hom}_{E_{*} \text { alg }}\left(T^{n+1}\left(E_{*} E\right), E_{*}\right) .
$$

Thus,

$$
C^{n} \simeq \operatorname{Hom}_{\left(E_{*}-\operatorname{alg} \downarrow E_{*}\right)}\left(T^{n+1}\left(E_{*} E\right), E_{*}[\epsilon] / \epsilon^{2}\right),
$$

and as we know from Sections 20 and 21, this complex computes $\operatorname{Der}_{E_{*}}^{s}\left(E_{*} E, E_{*+t}\right)$, which vanishes.

REMARK 23.8. Compare this to Robinson's calculation [15]. If we had taken $E \simeq K(n)$, the $n$th Morava $K$-theory, and if $p$ is odd (so that $K(n)$ is a commutative ring spectrum), then the proof of Proposition 23.7 shows that $H H^{s}\left(A, \pi_{0} \mathcal{E}_{E}^{S^{t}}\right)$ is the Quillen cohomology of the $K(n)$-algebra $K(n)_{*} K(n)$ with coefficients in the module $K(n)_{*+t}$. By a result of Quillen [12], these groups are the same up to a shift in dimension as the Hochschild cohomology of the algebra $K(n)_{*} K(n)$; this recovers Robinson's calculations of the obstruction groups for this spectrum.

The Hochschild cohomolgy of $K(n)_{*} K(n)$ is in general non 0 . However, the algebra $K(n)_{*} K(n) \simeq \Sigma(n) \otimes \Lambda(n)$, where $\Lambda(n)$ is an exterior algebra on $n$-generators, and the Hochschild cohomology of $K(n)_{*} K(n)$ originates almost entirely from this exterior factor. By replacing $K(n)$ with $E_{k, \Gamma}$ we get rid of this contribution.

Proof of Theorem 23.5. Let $C \cdot \rightarrow A$ be a cofibrant resolution of the operad $A$ by free operads; thus, $C_{n}=\mathcal{F} K_{n}$. We obtain a double complex $D_{m, n}=$ $\mathcal{B}\left(C_{n}, C_{n}, C_{n}\right)_{m}$ of bimodules using the bar construction. The diagonal of $D_{\bullet}, \bullet$ is a $C_{\bullet}$-bimodule resolving $C_{\bullet}$. If $N$ is a coefficient system for $A$, then by applying Hochschild cohomology we obtain a spectral sequence

$$
H H^{s}\left(C_{n}, N\right) \Longrightarrow H H^{s+n}(A, N) \text {. }
$$

Thus the theorem easily follows from the following lemma. 
Lemma 23.9. Let $C$ be a discrete operad. Then there is a sequence

$$
0 \rightarrow H H^{0}(C, N) \rightarrow H H^{0}(I, N) \rightarrow H^{0}(C, N) \rightarrow H H^{1}(C, N) \rightarrow 0
$$

natural in $C$; furthermore, if $C=\mathcal{F} K$ for some $\Sigma$-object $K$, the sequence is exact, and $H H^{s}(C, N) \simeq 0$ for $s>1$.

Proof. See [14].

\section{References}

1. J.F. Adams, Stable homotopy and generalised homology theories, University of Chicago Press, 1973.

2. A. K. Bousfield, Homotopy spectral sequences and obstructions, Israel J. Math. 66 (1989), $1-3$.

3. A. K. Bousfield and D. M. Kan, Homotopy limits, completions and localizations, Lecture Notes in Mathematics 304, Springer-Verlag, 1972.

4. V. G. Drinfel'd, Elliptic modules, Math. USSR-Sb. 23 (1974), 561-592.

5. A. D. Elmendorf, The Grassmanian geometry of spectra, Journal of Pure and Applied Algebra 54 (1988), 37-94.

6. A. D. Elmendorf, I. Kriz, M. A. Mandell, and J. P. May, Rings, modules, and algebras in stable homotopy theory, Mathematical Surveys and Monographs 47, Amer. Math. Soc., 1997.

7. A. Fröhlich, Formal groups, Lecture Notes in Mathematics 74, Springer-Verlag, 1968.

8. P. S. Landweber, Homological properties of comodules over $M U_{*}(M U)$ and $B P_{*}(B P)$, American Journal of Mathematics 98 (1976), 591-610.

9. L. G. Lewis, J. P. May, and M. Steinberger, Equivariant stable homotopy theory, Lecture Notes in Mathematics 1213, Springer-Verlag, 1986.

10. J. Lubin and J. Tate, Formal moduli for one-parameter formal lie groups, Bull. Soc. math. France 94 (1966), 49-60.

11. D. G. Quillen, Homotopical algebra, Lecture Notes in Mathematics 43, Springer-Verlag, 1967.

12. - On the (co-)homology of commutative rings, Applications of Categorical Algebra, Proceedings in the Symposia in Pure Mathematics, Amer. Math. Soc., 1970.

13. D. C. Ravenel, Complex cobordism and stable homotopy groups of spheres, Academic Press, 1986.

14. C. Rezk, Operads and spaces of algebra structures, PhD dissertation, MIT, 1995.

15. A. Robinson, Obstruction theory and the strict associativity of Morava K-theories, Advances in Homotopy Theory, Cambridge University Press, 1989, pp. 143-152.

16. J.-P. Serre, Local fields, Graduate Texts in Mathematics, Springer-Verlag, 1979.

17. J. H. Silverman, The arithmetic of elliptic curves, Graduate Texts in Mathematics, SpringerVerlag, 1986.

Department of Mathematics, Northwestern University, Evanston, IL 60208

E-mail address: rezk@math.nwu.edu 